\title{
A single cell atlas of human and mouse white adipose tissue
}

Margo P. Emont ${ }^{1,2}$, Christopher Jacobs ${ }^{1,2}$, Adam L. Essene ${ }^{1}$, Deepti Pant ${ }^{1}$, Danielle Tenen ${ }^{1,2}$, Georgia Colleluori ${ }^{3}$, Angelica Di Vincenzo ${ }^{3}$, Anja M. Jørgensen ${ }^{4}$, Hesam Dashti $^{2}$, Adam Stefek $^{2}$,

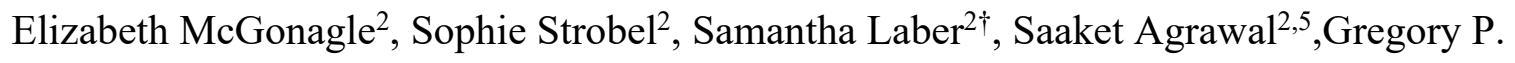

Westcott $^{1}$, Amrita Kar ${ }^{1,2}$, Molly L. Veregge ${ }^{1}$, Anton Gulko ${ }^{1}$, Harini Srinivasan ${ }^{1,2}$, Zachary

Kramer $^{1}$, Eleanna De Filippis ${ }^{1}$, Erin Merkel $^{1}$, Jennifer Ducie ${ }^{6}$, Christopher G. Boyd ${ }^{7}$, William Gourash $^{8}$, Anita Courcoulas ${ }^{8}$, Samuel J. Lin ${ }^{9}$, Bernard T. Lee ${ }^{9}$, Donald Morris ${ }^{9}$, Adam Tobias ${ }^{9}$, Amit V. Khera ${ }^{2,5,14}$, Melina Claussnitzer ${ }^{2,10}$, Tune H. Pers ${ }^{4}$, Antonio Giordano ${ }^{3}$, Orr Ashenberg ${ }^{11}$, Aviv Regev ${ }^{11,12,13}$, Linus T. Tsai ${ }^{1,2,14}$, Evan D. Rosen ${ }^{1,2,14}$

1. Division of Endocrinology, Diabetes and Metabolism, Beth Israel Deaconess Medical Center, Boston, MA, USA

2. Broad Institute of MIT and Harvard, Cambridge, MA, USA

3. Department of Experimental and Clinical Medicine, Center of Obesity, Marche Polytechnic University, Ancona, Italy.

4. Novo Nordisk Foundation Center for Basic Metabolic Research, University of Copenhagen, Copenhagen, Denmark

5. Center for Genomic Medicine, Department of Medicine, Massachusetts General Hospital, Boston, MA, USA

6. Division of Gynecologic Oncology, Department of Obstetrics and Gynecology, Beth Israel Deaconess Medical Center, Boston, MA, USA

7. Department of Surgery, Beth Israel Deaconess Medical Center, Boston, MA, USA 
8. Department of Surgery, University of Pittsburgh Medical Center, Pittsburgh, PA

9. Division of Plastic Surgery, Department of Surgery, Beth Israel Deaconess Medical Center, Boston, MA

10. Diabetes Unit and Center for Genomic Medicine, Massachusetts General Hospital, Boston, Massachusetts, 02114, USA.

11. Klarman Cell Observatory, Broad Institute of MIT and Harvard, Cambridge, MA, USA

12. Howard Hughes Medical Institute, Koch Institute of Integrative Cancer Research, Department of Biology, Massachusetts Institute of Technology, Cambridge, MA, USA

13. Genentech, South San Francisco, CA, USA

14. Harvard Medical School, Boston, MA

$\dagger$ Affiliated with Broad Institute while research was conducted

\section{Address correspondence to:}

Evan D. Rosen, MD PhD

Division of Endocrinology, Diabetes, and Metabolism

Beth Israel Deaconess Medical Center

330 Brookline Avenue

Boston, MA 02215

erosen@bidmc.harvard.edu 


\section{ABSTRACT}

2 White adipose tissue (WAT), once regarded as morphologically and functionally bland, is

3 now recognized to be dynamic, plastic, heterogenous, and involved in a wide array of

4 biological processes including energy homeostasis, glucose and lipid handling, blood

5 pressure control, and host defense ${ }^{1}$. High fat feeding and other metabolic stressors cause

6 dramatic changes in adipose morphology, physiology, and cellular composition ${ }^{1}$, and

7 alterations in adiposity are associated with insulin resistance, dyslipidemia, and type 2

8 diabetes (T2D) ${ }^{2}$. Here, we provide detailed cellular atlases of human and murine

9 subcutaneous and visceral white fat at single cell resolution across a range of body weight.

10 We identify subpopulations of adipocytes, adipose stem and progenitor cells (ASPCs),

11 vascular, and immune cells and demonstrate commonalities and differences across species

12 and dietary conditions. We link specific cell types to increased risk of metabolic disease,

13 and we provide an initial blueprint for a comprehensive set of interactions between

14 individual cell types in the adipose niche in leanness and obesity. These data comprise an

15 extensive resource for the exploration of genes, traits, and cell types in the function of WAT

16 across species, depots, and nutritional conditions.

18 A single cell atlas of human white adipose tissue

Mature adipocytes are too large and fragile to withstand traditional single cell approaches; as

20 a result, several groups have focused on the non-adipocyte stromal-vascular fraction (SVF) of

21 mouse $^{3-6}$ and human ${ }^{7}$ adipose tissue. An alternative strategy involves single nucleus (sNuc)

22 sequencing, which can capture adipocytes, and has been used to describe murine epididymal ${ }^{8,9}$

23 and human brown adipose tissue ${ }^{10}$. To compare these approaches in the context of human WAT, 
24 we pursued experiments on two cohorts of subjects. In the first, we collected subcutaneous WAT

25 from 9 women, isolated single cells from the SVF using collagenase digestion, and then

26 performed whole cell Drop-seq [hereafter referred to as single cell (sc)RNA-seq]. Because

27 different depots have been differentially linked to metabolic disease ${ }^{11}$, for the second cohort we

28 collected paired subcutaneous (SAT) and omental visceral (VAT) adipose tissue from 10

29 individuals, and SAT alone from three additional individuals (10 women, 3 men), and performed

30 sNuc-seq (Figures 1a, b, and Extended Data Table 1). Doublet and low-quality filtering left

31166,149 total cells $(28,465$ single cells and 137,684 single nuclei). The data from both

32 approaches were integrated, enabling the identification of the canonical cell types found in

33 WAT, including adipocytes, ASPCs, vascular cells, and immune cells (Figures 1c, d;

34 Supplementary Table 1). As expected, adipocytes were found only in the sNuc-seq dataset. The

35 sNuc-seq data was also enriched for vascular cells and macrophages, likely because collagenase

36 digestion did not fully dissociate these cell types. Mesothelial cells were not seen in the scRNA-

37 seq dataset, which did not include visceral tissue. Some of the visceral samples included cells

38 that appeared to be endometrial in origin $(P R L R+)$, likely due to endometriosis. Overall

39 proportions of adipocytes and ASPCs did not differ between depots, but depot clearly affects the

40 distribution of cells within these populations (Extended Data Figure 1a, b, 2a, b, Extended

41 Data Table 2). In our limited cohort, we could not detect major effects of BMI on cell type

42 proportions. To assess this finding at larger scale, we utilized our dataset as a reference to

43 estimate cell type proportions in bulk-RNA sequencing data ${ }^{12}$ obtained from the SAT of 331 men

44 in the METSIM cohort ${ }^{13}$. This deconvolution analysis found that the relative abundance of

45 adipocytes in that cohort was negatively correlated with BMI, while ASPCs and myeloid cells

46 were positively correlated (Figure 1e). 


\section{A single cell atlas of mouse white adipose tissue}

Murine models are commonly used to study adipose tissue biology ${ }^{14}$. We thus sought to compare mouse and human WAT at the single cell level by performing sNuc-seq on inguinal (ING, corresponding to human SAT) and perigonadal [PG, epididymal (EPI) in males, periovarian (POV) in females, corresponding to human VAT] adipose tissue of mice fed either a chow or high fat diet for 13 weeks (Figure 2a, b). After doublet removal and quality filtering, we considered a total of 197,721 cells (106,469 from PG and 91,252 from ING), identifying all cell types observed in human WAT (Figure 2c, d; Supplementary Table 2) with the addition of distinct male and female epithelial populations ( $D c d c 2 a+$ and Erbb4+, respectively). The female population is largely found in ING samples and resembles mammary epithelial cells, while the male population is almost exclusively found in PG samples, and as noted by others ${ }^{9}$ may represent contaminants from the epididymis and other reproductive structures that are tightly apposed to fat ${ }^{15}$. In contrast to the human data, cell type abundance in mouse WAT are highly

61 dependent on body weight with relatively little variation between depots (Figure 2c and

Extended Data Figure 3a, b, Extended Data Table 2). The proportions of cell types in mouse

63 adipose tissue after HFD were notably different between male and female mice, which might

64 reflect a true sex difference, or may reflect that males gain more weight on HFD (Extended

65 Data Figure 3b). To compare across species, we used a reference mapping algorithm to assign each mouse cell to a human cluster and noted a high degree of overall similarity between annotated mouse clusters and mapped human clusters (Extended Data Figure 3c). Similarly, the proportions of each cell type were roughly similar between humans and chow-fed mice (compare 


\section{Analysis of human and mouse stromal-vascular cellular subtypes}

\section{Vascular Cells}

73 Subclustering of human vascular cells revealed expected cell types including blood

74 endothelial clusters that represent arteriolar, stalk, and venular cells, as well as lymphatic

75 endothelial cells (LECs), pericytes, and two distinct populations of smooth muscle cells (SMCs)

76 (Extended Data Figure 4a, b). Mouse vascular cells formed similar clusters, but with only one

77 SMC cluster (Extended Data Figure 4c, d). As expected, reference mapping demonstrated high

78 similarity between human and mouse vascular subclusters (Extended Data Figure 4e). The

79 proportions of vascular cells were similar across depots for both mouse and human, although

80 LECs were to be more common in visceral fat of both species (Extended Data Figure 4f, $\mathbf{g}$ ).

81 There was little effect of adiposity on vascular cell populations in the human samples; mice,

82 however, showed significant changes in vascular cells after high fat feeding, including a lower

83 proportion of $\mathrm{Dkk2}^{+}$arteriolar cells and concomitantly higher levels of venular cells. There was

84 also a reduction in the relative proportion of LECs and increased pericytes on HFD (Extended

\section{Data Figure 4f, g).}

\section{Immune Cells}

Analysis of human immune cells from scRNA-seq and sNuc-seq samples again revealed expected cell types, including multiple subpopulations of monocytes, macrophages $(C D 14+)$,

90 dendritic cells (DCs), B and T lymphocytes, and NK cells (CD96+), as well as mast cells

$91(C P A 3+)$ and neutrophils $(C S F 3 R+)($ Extended Data Figure 5a, b). These subpopulations

92 resemble known immune cell populations. For example, monocyte subpopulations 1 and 2 
93 resemble classical and non-classical monocytes and DC subpopulations 1 and 2 similarly

94 resemble previously reported $C L E C 9 A+$ and $C D 1 C+$ populations from blood, respectively ${ }^{16}$.

95 Lymphocytes also resemble previously reported B cell, T Cell, and NK cell populations from

96 human WAT, including CTLA4+ $\operatorname{hTregs}^{17}$. Examination of the mouse WAT immune

97 compartment revealed most of the same cell types, although there were notable differences in the

98 relative abundance of myeloid and lymphoid cells between species (Extended Data Figure 5c,

99 d). Human WAT contains somewhat fewer T/NK cells than macrophages/monocytes ( $\sim 30 \%$ vs.

$100 \sim 60 \%$ of recovered immune cells); this imbalance was greatly exaggerated in murine WAT

101 (macrophages $\sim 90 \%$ of recovered immune cells vs. $3 \%$ T/NK cells). Because a wealth of data

102 supports a key role for macrophages/monocytes in adipose biology ${ }^{18,19}$, we separated these cell

103 types from other immune cells in silico for subsequent analysis. Mouse clusters of non-

104 monocytes/macrophages mapped relatively well to their human counterparts, with some mixing

105 of T and NK populations (Extended Data Figure 5e). Macrophages and monocytes also mapped

106 well to their general class, but this association often broke down when considering macrophage

107 subpopulations (Extended Data Figure 5f). Thus, mouse cluster mMac3, which comprises the

$108 \operatorname{Trem}^{+}$cells also called "lipid-associated" macrophages ${ }^{1}$ maps well to $T R E M 2^{+}$human hMac2

109 cells, as expected, but hMac2 also associated with every other mouse macrophage subpopulation,

110 most notably the $\mathrm{Fgfl} 3^{+}$mMac1 group (Extended Data Figure 5f).

111 The proportion of immune cell populations was similar in human SAT and VAT, with a few

112 exceptions, such as $P R O S 1^{+}$hMac3 cells which were more abundant in VAT (Extended Data

113 Figure 6a, e). In mice, small depot-dependent differences were eclipsed by relatively huge shifts

114 in response to diet in male mice (Extended Data Figure 6b, d, f). Most notably, HFD resulted

115 in a massive increase in macrophage numbers, primarily in PG, consistent with a large body of 
116 prior data ${ }^{18,21}$, (Extended Data Figure 3c, $6 \mathbf{6}$ ). As a proportion of total immune cells, HFD

117 induced large shifts in mMac1 (down in ING, up in EPI), mMac2 (down in EPI), and Trem2 $2^{+}$

118 mMac3 (up in ING and EPI) in male mice (Extended Data Figure 6f). Reductions in the

119 proportion of most other immune cell types (e.g., NK cells, T and B lymphocytes, DCs, and

120 neutrophils) are likely due to the large influx of macrophages, rather than to intrinsic loss of

121 those specific cell types following HFD (Extended Data Figure 6b, d, f). Mast cells increase

122 proportionally after HFD despite the influence of macrophages, as previously reported ${ }^{22}$. Female

123 mice exhibit a much less impressive response to HFD, with the only significantly different diet-

124 related change being a reduction in $\operatorname{Prg} 4+$ mMac4 cells (Extended Data Figure 6f).

125 Accumulation of adipose tissue macrophages in obesity has also been shown in human WAT,

126 using a combination of histomorphometry and flow sorting ${ }^{19,23}$. Our data are in general support

127 of this conclusion, though the magnitude of the effect is significantly less prominent than that

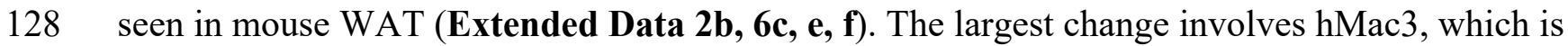

129 induced in visceral fat with higher BMI (Extended Data 6c, e). We did not observe differential

130 representation of other immune cell in WAT from subjects with high BMI vs. low BMI.

\section{Mesothelial cells}

133 Subclustering of mesothelial cells revealed three populations in both human VAT and mouse

134 PG (Extended Data Figure 7a-d). Only sNuc-seq samples were used in this analysis because

135 our human scRNA-seq data did not include VAT. When mouse mesothelial clusters were

136 mapped to human clusters, cells were split between human clusters hMes1 and hMes2, with no

137 cells mapping to hMes3 (Extended Data Figure 7e). The proportions of most mesothelial

138 subpopulations did not vary with obesity or high fat diet, with the exception of hMes1 and 
139 hMes2, which were reduced and increased in higher BMIs, respectively. (Extended Data Figure

$1407 \mathbf{7}, \mathbf{g})$.

$A S P C s$ (see Supplementary Note 1)

143 We identified six distinct subpopulations of human ASPCs in subclustered scRNA-seq and

144 sNuc-seq samples, all of which express the common marker gene PDGFRA (Extended Data

145 Figure 8a, b). Similarly, we noted six subpopulations in the mouse ASPC data, all of which

146 were also $\mathrm{Pdgfra}+$ and some of which correspond well with a particular human subpopulation

147 (Extended Data Figure 8c-e). For example, mASPC2 and hASPC2 are both characterized by

148 high expression of Aldhla3/ALDH1A3, and strongly resemble previously identified early

149 multipotent progenitor cells that reside in the reticular interstitium of the fat pad ${ }^{5}$. Similarly,

$150 \mathrm{mASPC} 4$ and hASPC4 express Epha3/EPHA3 and likely represent the anti-adipogenic Areg

151 population reported by Schwalie et. al. ${ }^{3}$. Seeking to better place our mouse ASPC data into the

152 overall context of the published literature, we performed reference mapping between our ASPCs

153 and ASPC populations reported by others ${ }^{3-6,9}$ and found general agreement across studies

154 (Extended Data Figure 8f). As mentioned, mASPC2 cells map to the Dpp $4+/ E b f 2+$ ASPCs

155 identified by other studies and mASPC1 and mASPC6 map strongly to adipose progenitors,

156 including the $\operatorname{Icam} 1^{+}$cells identified by Merrick et. al. ${ }^{5}$.

157 Many human and mouse ASPC subclusters showed dependency on diet, depot, or both.

158 hASPC1, hASPC4, and hASPC5 were more prevalent in SAT than VAT, with increases in SAT

159 hASPC4 and hASPC5 proportion in subjects with higher BMI (Extended Data Figure 9a, c, e).

160 Conversely, hASPC3 and hASPC6 were more prevalent in VAT. In male mice, early progenitor

161 cells (mASPC2) were notably more abundant in ING than PG; such depot selectivity was not 
162 noted for the analogous hASPC2 in humans. mASPC5 and mASPC6 were more prevalent in EPI

163 vs ING, although this varied with obesity (e.g., the proportion of mASPC6 cells was greater in

164 EPI than ING, but only after HFD) (Extended Data Figure 9b, d, f). Many of these

165 observations are consistent with previous findings in adipose biology. For example, HFD has

166 been shown to increase adipogenesis specifically in PG in mice ${ }^{24,25}$. Our data indicates that pre-

167 adipocyte subclusters like mASPC6 increase dramatically in response to HFD in PG only. The

168 loss of early progenitors (mASPC2) in PG with HFD is consistent with conversion of these cells

169 along the differentiative pathway, i.e., toward mASPC6 (Extended Data Figure 9b, d, f). These

170 patterns are harder to discern in the human samples, which may reflect the fact that patient data

171 are captured at variable time points after the onset of obesity, whereas the mouse samples are

172 synchronized over a relatively short time period. Nonetheless, we do observe a VAT-specific

173 increase in hASPC6 in subjects with high BMI $(\mathrm{BMI}>40)($ Extended Data Figures 8e, 9e).

\section{Unique subpopulations of human white adipocytes}

176 White adipocytes are generally considered to be monotypic and essentially uniform in

177 function, although some recent studies have begun to challenge this assumption ${ }^{8-10,26}$ The high

178 resolution of our data enabled us to find that human white adipocytes cluster into seven

179 subpopulations with distinct markers (Figure 3a-b). We noted strong depot-specific associations

180 of adipocyte subtypes, with hAd1, hAd3, hAd4, and hAd7 localized primarily to SAT, while

181 hAd2 and hAd6 were almost exclusively found in VAT. hAd5 represents a smaller population

182 that is roughly equally distributed between SAT and VAT (Extended Data Figure 10a-c). We

183 also noted a BMI-dependent shift in adipocyte subtype within both depots (Extended Data

184 Figure 10b, c). Importantly, all adipocyte subpopulations are present in the majority of subjects, 
185 indicating that these subtype designations are generalizable and do not reflect sample-specific

186 variation (Extended Data Figure 10c). Immunohistochemistry (IHC) and/or

187 immunofluorescence of markers for hAd4, hAd5, hAd6, and hAd7 in human subcutaneous or

188 visceral adipose tissue identified specific subpopulations of adipocytes at proportions similar to

189 those seen in the single cell data (Figure 3c and Extended Data Figure 10 d, e). To examine

190 whether SAT subtype proportion was influenced by BMI in a larger dataset, we estimated

191 individual subtype proportions by deconvolution analysis of bulk RNA-seq data from purified

192 isolated subcutaneous human adipocytes from 43 women (Figure 3d). This analysis showed that

193 clusters hAd4 and hAd7 trend to negative correlation with BMI, aligning with our IHC findings,

194 while hAd5 proportion is positively correlated with BMI. Visceral adipocytes are absent from

195 this dataset and so we were unable to assess the prevalence of hAd2 or hAd6 in this cohort,

196 although IHC of hAd6 marker EBF2 also suggests its prevalence may be positively correlated

197 with BMI (Figure 3c).

198 A critical question is whether individual adipocyte subpopulations have specific functions.

199 To assess this, we first looked at genes that participate in the major metabolic activities of

200 adipocytes, including adipokine synthesis and secretion, insulin signaling, lipid handling, and

201 thermogenesis. All subpopulations expressed these genes, although their relative levels differed.

202 Thus, the adipokines adiponectin and adipsin (CFD) are most highly expressed in hAd3, and

203 insulin signaling components like INSR, IRS1 and IRS2 are most highly expressed in hAd5

204 (Extended Data Figure 10f). We next looked more holistically at the data by performing 205 pathway analysis for markers of each subpopulation (Supplementary Table 3, Extended Data

206 Figure 10g-m). Subpopulations hAd1, which accounts for $\sim 40 \%$ of SAT adipocyte nuclei, and

207 hAd2, which accounts for $\sim 60 \%$ of VAT adipocyte nuclei, have relatively few specific markers, 
and the pathways that emerged were similarly unrevealing (Extended Data Figure 10g, h).

209 These populations likely represent "basal" subcutaneous or visceral adipocytes, so we therefore

210 focused on subpopulations hAd3-hAd7 for more detailed analysis. hAd3, which comprises $\sim 15 \%$

211 of VAT, was associated with "triglyceride biosynthesis" and included higher expression of

212 DGAT2, SREBF1, and PNPLA3 (Extended Data Figure 10i). The hAd4 cluster, which makes

213 up $\sim 40 \%$ of SAT, expresses the highest levels of several fatty acid desaturases, including

214 ELOVL5 and FADS3 (Extended Data Figure 10j), which is particularly interesting in light of

215 the insulin-sensitizing role of unsaturated lipokines such as palmitoleate ${ }^{27}$. hAd5 adipocytes

216 comprise a relatively small amount of both SAT and VAT, and besides having the highest

217 expression of several insulin signaling genes, were also characterized by expression of

218 “sphingolipid signaling genes" (Extended Data Figure 10k). Both hAd3 and hAd4 express high

219 levels of lipogenic genes, while hAd5 expresses higher levels of lipolysis genes (Extended Data

220 Figure 10f).

221 We next asked whether cultured human adipocytes retain evidence of subpopulation

222 diversity. To that end, we utilized 57 RNA-seq datasets from human subcutaneous and visceral

223 adipocyte progenitors differentiated ex vivo over a 14 day timecourse ${ }^{28}$. Deconvolution analysis

224 revealed that many subpopulations identified in vivo were retained in the dish. Furthermore,

225 much of the previously noted depot selectivity was recapitulated, such that the visceral

226 subpopulations hAd2 and hAd6 were significantly more likely to appear in cultured visceral cells

227 and the subcutaneous subpopulation hAd4 was overrepresented in cultured subcutaneous cells

228 (Extended Data Figure 11a). Furthermore, because these cultured samples were also subjected

229 to high-content image-based profiling using LipocyteProfiler ${ }^{28}$, we were able to correlate

230 individual subpopulations with image-based features representing morphological and cellular 
231 phenotypes including lipid and mitochondrial content. Thus, ex vivo differentiated adipocyte

232 cultures predicted to have high amounts of hAd3, which express high levels of lipogenic genes

233 and lower levels of lipolytic genes have more overall lipid and larger lipid droplets (Figure 3e,

234 f). Conversely, ex vivo differentiated adipocyte cultures with high predicted hAd5 content have

235 less overall lipid and smaller lipid droplets, consistent with higher expression of lipolytic genes

236 and less lipogenic gene expression (Extended Data Figure 11b-d).

237 One particularly interesting adipocyte subpopulation is hAd6, which selectively expresses

238 genes typically associated with thermogenesis, such as EBF2, ESRRG, and PPARGC1A

239 (Extended Data Figure 101), a surprising finding given that this population is almost

240 exclusively visceral (Figure 3c, Extended Data Figure 10c). To better understand the

241 relationship between this subpopulation and visceral adiposity, we looked further into the hAd6

242 marker EBF2, which has previously been identified as a pro-thermogenic transcription factor ${ }^{29}$.

243 SNPs at the EBF2 locus are associated with waist-hip ratio (WHR) ${ }^{30}$, which could involve

244 actions in either SAT or VAT. Interestingly, however, a recent study of GWAS loci associated

245 with adiposity in specific depots ${ }^{31}$ found a common variant $15 \mathrm{~kb}$ upstream of EBF2 that was

246 associated specifically with VAT (Extended Data Figure 12a). Further analysis revealed that

247 the minor allele of this SNP $(\mathrm{MAF}=0.23)$ was associated with VAT adjusted for BMI and

248 height $\left(\right.$ VATadj: beta $=0.062$ SD per allele, $\left.p=1.0 \times 10^{-12}\right)$, but not abdominal subcutaneous

249 (ASAT) or gluteofemoral (GFAT) depots (ASATadj: beta $=-0.018$ SD per allele, $p=0.03$ ),

250 GFATadj: beta $=-0.020$ SD per allele, $p=0.02$, Extended Data Figure 12b). We additionally

251 stratified individuals into either 0,1 , or 2 carriers of the minor allele and observed an additive

252 trend (G/G median VATadj -0.10 SD, G/A median VATadj = -0.04 SD, A/A median VATadj

253 0.04 SD; Extended Data Figure 12c). Next, we returned to the visceral human adipocytes 
254 differentiated ex vivo, and found that samples predicted to have a higher proportion of hAd6

255 adipocytes were characterized by higher mitochondrial intensity and increased expression of

256 mitochondrial and thermogenic genes (Extended Data Figure 12d-f). Finally, our analysis of

257 hAd6 markers suggested other pathways associated with thermogenesis, including one for "axon

258 guidance" (Extended Data Figure 12g). We could not measure innervation directly using our

259 data, because the nuclei of innervating sympathetic neurons are located in the spinal ganglia and

260 not the fat depot itself. Nonetheless, we estimated relative levels of innervation using the

261 presence of neuron-specific gene expression in the ambient RNA of our visceral sNuc-seq

262 samples. Indeed, the amount of pan-neuronal markers like TUBB3 ( $\beta$ III-tubulin) and UCHL1

263 (PGP9.5) ${ }^{32}$ strongly correlate with hAd6 proportion (Extended Data Figure 12e), further

264 supporting a role for hAd6 as a novel visceral adipocyte subtype with thermogenic potential.

\section{Adipocytes of mice and humans show critical similarities and differences}

267 Subclustering mouse adipocytes revealed six subpopulations (Figure 3g, h). Unlike human

268 adipocytes, mouse adipocyte subtypes exhibit little depot enrichment, especially on chow diet

269 (Extended Data Figure 13a-c). There was strong diet-dependency, however, as relative

270 proportions of mAd1 and mAd3 were reduced after HFD, while the opposite was noted for

271 mAd4 and mAd5 (Extended Data Figure 13b, c). In contrast to the relatively good cross-

272 species concordance between immune cells, vascular cells, and ASPCs, mouse adipocytes do not

273 map cleanly onto human adipocyte subpopulations. The majority of murine ING adipocytes map

274 most closely to hAd1, while PG adipocytes map to hAd6, with some mapping to hAd2.

275 (Extended Data Figure 13d-f). 
As in the human, genes associated with major adipocyte functions showed some

population mAd5 (Extended Data Figure 13c, g). More detailed pathway analysis on mouse adipocyte subpopulations (Supplementary Table 3) showed that the chow-associated clusters mAd1-3 were notably enriched in metabolic pathways, particularly those involved in lipid

281 handling (Extended Data Figure 13h-j). The HFD-associated clusters mAd4-6, on the other

282 hand, were linked to pathways like "HIF-1 signaling”, “actin cytoskeleton", and "NF-кB

283 signaling” (Extended Data Figure 13k-n), consistent with the known roles of hypoxia,

284 cytoskeletal remodeling, and inflammation in HFD-induced adipose dysfunction and insulin 285 resistance ${ }^{23-25}$.

286 Our data allows us to address an important question: are diet-induced changes in gene expression at the population level shared among subpopulations or do they reflect a change in the relative proportion of these subpopulations? To assess this, we examined the twenty most positively and negatively regulated genes from a TRAP-based RNA-seq experiment in white 290 adipocytes from mice fed chow or high fat $\operatorname{diet}^{34}$ (Extended Data Figure 14a). We noted that 291 some genes, such as Cyp2e1, and Fam13a, exhibit elevated expression in chow adipocytes in 292 virtually all subpopulations, even those clusters that are selective for HFD (Extended Data 293 Figure 14b). However, while the chow-associated gene $C f d$ is reduced in all populations with 294 HFD, expression seems largely driven by the mAd3 population which has the highest expression 295 of $C f d$ and decreases in abundance with HFD (Extended Data Figure 13b,c, 14b). Sept 9 , $296 \mathrm{Cdknla}$, and Fgfl3 show increased gene expression after HFD across almost all subpopulations 297 while other HFD-induced genes (e.g., Slc5a7 and Dclk1) increase their expression after HFD in 298 the chow-associated clusters (mAd1-4) but not in the HFD-associated clusters mAd5-7 
(Extended Data Figure 14b). Thus, diet-dependent expression changes reflect both alterations across all clusters and the emergence or disappearance of distinct populations.

Finally, we were somewhat surprised that we did not see a murine population that could be

302 clearly delineated as thermogenic. Such cells have been noted by others in WAT, even at room

303 temperature ${ }^{36}$. Notably, the distribution of beige adipocytes is not uniform in ING, but tends to

304 be densest close to the inguinal lymph node $(\mathrm{LN})^{37}$. To avoid contamination by LN cells, we

305 excised the node with a fairly wide margin, and it is possible that our samples were thus de-

306 enriched for beige adipocytes. Nonetheless, when we considered the chow fed samples

307 independently, mAd1 split into three clusters (Extended Data Figure 15a, b). Two of these

308 clusters, mAd1B and mAd1C, were recognizable as thermogenic beige adipocytes, with

309 relatively high expression of Prdm16 and Ppargcla in mAd1B and even higher expression of

310 these genes, as well as expression of Ucpl and Cidea in mAd1C (Extended Data Figure 15c).

311 As expected, the thermogenic mAd1B and mAd1C subpopulations were enriched in ING vs. PG

312 samples (Extended Data Figure 15d, e) and suggest HFD-induced transcriptional variability

313 masks these subtype designations.

\section{Exploration of cell-cell interactions within the adipose niche}

316 The functions of WAT are known to be coordinated by neural and hormonal cues from

317 outside the fat $\operatorname{pad}^{38}$. There is growing appreciation, however, that intercellular communication

318 within the depot is also critical for the WAT response to overnutrition and other stressors ${ }^{39}$. In

319 particular, attention has focused on cross-talk between adipocytes and immune cells (especially

320 macrophages) in the context of obesity ${ }^{40}$. To assess potential interactions between all identified

321 cell types in different depots and at different body mass, we utilized CellPhoneDB ${ }^{41}$, which 
322 utilizes information about the expression of ligand-receptor pairs to estimate cell type

323 communication (Supplementary Table 4, Supplementary Table 5). As expected, we detected

324 increased potential communication between human adipocytes and macrophages in high BMI vs.

325 low BMI subjects; of 84 potential interactions identified between human adipocytes and

326 macrophages, $40(48 \%)$ were specific for high BMI subjects, while only $3(4 \%)$ were specific for

327 low BMI subjects (Figure 4a, Extended Data Figure 16a, d). Notably, obesity was also

328 associated with robustly increased expression of genes encoding ligand-receptor pairs between

329 adipocytes and many non-immune cell types, including blood and lymphatic endothelial cells,

330 vascular SMCs, pericytes, and ASPCs (Figure 4a, b, Extended Data Figure 16a, d). For

331 example, of 145 potential interactions identified between human adipocytes and endothelial

332 cells, $65(45 \%)$ were specific for high BMI subjects, while only $6(4 \%)$ were specific for low

333 BMI subjects (Extended Data Figure 16d). Potential interactions between these cell types are

334 frequently bidirectional, and receptors are often expressed on multiple cell types, suggesting

335 networks of communication (Figure 4b, Extended Data Figure 16e). We also noted differential

336 expression of ligands and receptors within human adipocyte subpopulations, lending further

337 support to the idea that they carry out distinct functions (Extended Data Figure 16b). The

338 specific interactions upregulated during obesity suggest that adipocytes play a significant role in

339 obesity-related adipose tissue remodeling. For example, adipocyte expression of angiogenic

340 factors like $J A G 1$ and $V E G F C$ is increased in the obese state, as is true of the expression of their

341 receptors (e.g., $N O T C H 3$ and $K D R$ ) on endothelial cells, consistant with obesity-associated

342 induction of angiogenesis by adipocytes ${ }^{42}$ (Figure 4b, Supplementary Table 6).

343 Analysis of the mouse data yielded similar results, as HFD increased the intensity of ligand-

344 receptor pair expression, with the most prominent interactions again occurring between non- 
345 immune cell types, especially between ASPCs and adipocytes, pericytes, and SMCs (Extended

346 Data Figure 16c). Interactions between WAT cell types include several that have been studied,

347 such as the effect of the adipokine leptin on endothelial cells via LEPR ${ }^{43}$, and the actions of

348 TGFB1 on adipose fibrosis via TGFBR $1^{34}$. The majority of these interactions, however, are

349 unstudied in the context of WAT function and dysfunction.

350 In human samples, most interactions between adipocytes and endothelial cells were shared

351 between SAT and VAT (61\%), but of those interactions not shared between depots, the majority

352 were seen in SAT (31\% vs. 8\% specific for VAT). This same pattern was seen when looking at

353 adipocyte-ASPC interactions (38\% SAT-specific vs. 11\% VAT-specific), and adipocyte-

354 macrophage interactions (27\% SAT-specific vs. 12\% VAT-specific). In mice, we noted a more

355 even split between ING- and EPI-specific interactions (e.g., 13\% ING-specific vs. 12\% EPI-

356 specific adipocyte-endothelial interactions). Adipose niche interactions were only modestly

357 conserved between mouse and human. (Extended Data Figure 16d).

Relationships between WAT cell types and human disease

Adiposity is associated with a wide range of metabolic diseases and traits, and GWAS

360 studies have suggested a specific link between WAT and coronary artery disease (CAD), BMI-

361 adjusted T2D, dyslipidemia, and BMI-adjusted waist-hip ratio (WHR, a measure of body fat

362 distribution $)^{44-46}$. To determine which specific cell types in WAT are likely to mediate these

363 associations, we employed CELLECT, a method for integrating scRNA-seq and sNuc-seq data

364 with GWAS ${ }^{47}$. As expected, Type 1 Diabetes (T1D) was significantly associated with B and T

365 lymphocytes and NK cells, consistent with the known autoimmune basis of that disease (Figure

366 4c). No WAT cell type associated with BMI, as expected given the strong neuronal basis of body

367 weight regulation ${ }^{48}$. The strongest phenotypic association for white adipocytes was with BMI- 
adjusted WHR, and associations approaching significance were also noted between adipocytes and HDL and T2D (Figure 4c, Supplementary Table 7).

Because all adipocyte subpopulations were significantly associated with WHR (Figure 4d), we looked for adipocyte genes responsible for the association with WHR that are not specific to any particular subpopulation. One such gene is $P P A R G$, which is highly expressed in all

373 adipocytes (Extended Data Figure 17a). Data from the METSIM cohort indicates a strong

374 inverse relationship between WHR and $P P A R G$ levels in whole WAT (Extended Data Figure 17b). Unfortunately, WHR was not recorded in the cohort used to generate our floated human adipocytes. WHR is, however, highly correlated with HOMA-IR ${ }^{11}$, and we found that PPARG levels showed a strong inverse relationship with HOMA-IR in both the METSIM cohort and in our floated adipocytes (Extended Data Figure 17c, d). Furthermore, SNPs in the PPARG gene that are associated with BMI-adjusted $\mathrm{WHR}^{30}$ are also significantly associated with $P P A R G$ mRNA levels and HOMA-IR in our floated adipocyte cohort (Extended Data Figure 17e-h).

Adipocytes were also the cell type most likely to mediate the association of WAT with T2D, with the strongest association specifically with hAd7 (Figure 4d). To further investigate the association between hAd7 and T2D, we took our deconvolved bulk RNA-seq data from floated human adipocytes and plotted the abundance of hAd7 as a function of HOMA-IR. This revealed that hAd7 shows significant inverse correlation with insulin resistance (Figure 4e). We then searched for specific hAd7 marker genes that exhibit this same relationship with HOMA-IR, and

387 identified several, including $A G M O, A L P K 3, F H O D 3$, and LIN7A (Figure 4f, g). Of note,

388 AGMO (also called TMEM195) has emerged as a candidate locus in T2D GWAS ${ }^{49,50}$.Taken

389 together, our data suggest that hAd7 may have an outsized influence on the risk of T2D, despite

390 representing only $\sim 1 \%$ of human adipocytes. 
Additionally, although adipocytes did not meet genome-wide significance for an association with LDL, we were struck by the near significant relationship between LDL and hAd1, and to a

393 lesser extent, hAd4 (Figure 4c, d). We noted several genes that were selective for hAd1 and/

394 hAd4, including NRCAM, PEMT, PCDH7, and VGLL3, all of which showed a strong positive

395 relationship between expression and LDL levels in our floated adipocyte cohort (Extended Data

396 Figure 17i, j)

397 We also performed CELLECT using the mouse data and noted associations between BMI398 adjusted WHR and murine adipocytes (particularly mAd1, mAd3, and mAd6), as well as pre399 adipocytes (especially mASPC2) (Extended Data Figure 18a-c). This suggests that WHR may

400 be determined in large part by alterations in adipocyte differentiation, a hypothesis consistent 401 with the PPARG data above, and with independent studies of different WHR genes ${ }^{51}$. HDL and 402 TG levels are also associated with mouse white adipocyte gene expression (Extended Data 403 Figure 18a-c).

\section{Discussion}

Here, we present a comprehensive atlas of human and mouse WAT across depot and

407 nutritional state. Our analysis reveals a rich array of cell types, including blood and lymphatic

408 vascular cells, immune cells, and ASPCs, in addition to adipocytes. These cell types are grossly

409 similar across species, but differ more profoundly when cellular subpopulations are explored. It

410 is tempting to attribute these subpopulation differences to divergence across 65 million years of

411 evolution, but other factors also need to be considered. For example, the human samples were

412 collected after a fast, while the mice were harvested after ad libitum feeding, which might be

413 expected to cause some differences in cell state related to insulin signaling or related pathways. 
414 Ongoing studies are focused on addressing potential effects of fasting/feeding on WAT

\section{5 composition.}

416 Our dataset reveals subpopulations of human white adipocytes that are associated with a

417 range of adipocyte functions, from lipolysis and lipogenesis to thermogenesis, as well as with

418 phenotypes such as BMI, WHR, and T2D. The single cell resolution of our dataset enables the

419 identification of heterogeneity that cannot be appreciated by bulk RNA sequencing, such as a

420 potentially visceral thermogenic subpopulation (hAd6), and a rare subpopulation associated with

421 T2DM (hAd7). Our dataset provides a rich resource to identify other disease-associated cell

422 types and to better interpret GWAS studies of metabolic phenotypes.

423 Overall, our data highlight a central role for adipocytes in the local regulation of the adipose

424 depot as well as in systemic physiology. We additionally provide a framework for mouse-human

425 comparison in studies of adipose tissue that will be an important resource for groups hoping to

426 translate murine findings to human treatments. These data provide a lens of unprecedented acuity

427 that better informs our understanding of WAT biology and enables a deeper exploration of the

428 role of adipose tissue in health and disease. 


\section{REFERENCES}

429 1. Rosen, E. D. \& Spiegelman, B. M. What We Talk About When We Talk About Fat. Cell 156, $430 \quad 20-44(2014)$.

431 2. Kahn, S. E., Hull, R. L. \& Utzschneider, K. M. Mechanisms linking obesity to insulin

432 resistance and type 2 diabetes. Nature 444, 840-846 (2006).

433 3. Schwalie, P. C. et al. A stromal cell population that inhibits adipogenesis in mammalian fat 434 depots. Nature 559, 103-108 (2018).

435 4. Burl, R. B. et al. Deconstructing Adipogenesis Induced by $\beta 3$-Adrenergic Receptor Activation 436 with Single-Cell Expression Profiling. Cell Metab. 28, 300-309.e4 (2018).

437 5. Merrick, D. et al. Identification of a mesenchymal progenitor cell hierarchy in adipose tissue.

$438 \quad$ Science 364, (2019).

439 6. Hepler, C. et al. Identification of functionally distinct fibro-inflammatory and adipogenic

440 stromal subpopulations in visceral adipose tissue of adult mice. eLife 7, e39636 (2018).

441 7. Vijay, J. et al. Single-cell analysis of human adipose tissue identifies depot- and disease$442 \quad$ specific cell types. Nat. Metab. 2, 97-109 (2020).

443 8. Rajbhandari, P. et al. Single cell analysis reveals immune cell-adipocyte crosstalk regulating 444 the transcription of thermogenic adipocytes. eLife 8, e49501 (2019).

445 9. Sárvári, A. K. et al. Plasticity of Epididymal Adipose Tissue in Response to Diet-Induced 446 Obesity at Single-Nucleus Resolution. Cell Metab. 33, 437-453.e5 (2021).

447 10. Sun, W. et al. snRNA-seq reveals a subpopulation of adipocytes that regulates 448 thermogenesis. Nature 587, 98-102 (2020). 
449 11. Benites-Zapata, V. A. et al. High waist-to-hip ratio levels are associated with insulin

$450 \quad$ resistance markers in normal-weight women. Diabetes Metab. Syndr. Clin. Res. Rev. 13, 636-

$451 \quad 642(2019)$.

452 12. Wang, X., Park, J., Susztak, K., Zhang, N. R. \& Li, M. Bulk tissue cell type

453 deconvolution with multi-subject single-cell expression reference. Nat. Commun. 10, 380

$454 \quad$ (2019).

455 13. Raulerson, C. K. et al. Adipose Tissue Gene Expression Associations Reveal Hundreds

456 of Candidate Genes for Cardiometabolic Traits. Am. J. Hum. Genet. 105, $773-787$ (2019).

457 14. Blüher, M. Transgenic animal models for the study of adipose tissue biology. Best Pract.

458 Res. Clin. Endocrinol. Metab. 19, 605-623 (2005).

459 15. An atlas of cell types in the mouse epididymis and vas deferens $\mid$ eLife.

460 https://elifesciences.org/articles/55474.

461 16. Villani, A.-C. et al. Single-cell RNA-seq reveals new types of human blood dendritic 462 cells, monocytes, and progenitors. Science 356, (2017).

463 17. Hildreth, A. D. et al. Single-cell sequencing of human white adipose tissue identifies new 464 cell states in health and obesity. Nat. Immunol. 1-15 (2021) doi:10.1038/s41590-021-00922-4.

465 18. Suganami, T. \& Ogawa, Y. Adipose tissue macrophages: their role in adipose tissue 466 remodeling. J. Leukoc. Biol. 88, 33-39 (2010).

467 19. Weisberg, S. P. et al. Obesity is associated with macrophage accumulation in adipose 468 tissue. J. Clin. Invest. 112, 1796-1808 (2003).

469 20. Jaitin, D. A. et al. Lipid-Associated Macrophages Control Metabolic Homeostasis in a 470 Trem2-Dependent Manner. Cell 178, 686-698.e14 (2019). 
471 21. Reilly, S. M. \& Saltiel, A. R. Adapting to obesity with adipose tissue inflammation. Nat.

472 Rev. Endocrinol. 13, 633-643 (2017).

473 22. Shi, M. \& Shi, G.-P. Different Roles of Mast Cells in Obesity and Diabetes: Lessons

474 from Experimental Animals and Humans. Front. Immunol. 3, 7 (2012).

475 23. Xu, H. et al. Chronic inflammation in fat plays a crucial role in the development of

476 obesity-related insulin resistance. J. Clin. Invest. 112, 1821-1830 (2003).

477 24. Wang, Q. A., Tao, C., Gupta, R. K. \& Scherer, P. E. Tracking adipogenesis during white 478 adipose tissue development, expansion and regeneration. Nat. Med. 19, 1338-1344 (2013).

479 25. Jeffery, E., Church, C. D., Holtrup, B., Colman, L. \& Rodeheffer, M. S. Rapid Depot480 Specific Activation of Adipocyte Precursor Cells at the Onset of Obesity. Nat. Cell Biol. 17, $481 \quad 376-385$ (2015).

482 26. Bäckdahl, J. et al. Spatial mapping reveals human adipocyte subpopulations with distinct 483 sensitivities to insulin. Cell Metab. 33, 1869-1882.e6 (2021).

484 27. Stefan, N. et al. Circulating Palmitoleate Strongly and Independently Predicts Insulin 485 Sensitivity in Humans. Diabetes Care 33, 405-407 (2010).

486 28. Laber, S. et al. Discovering cellular programs of intrinsic and extrinsic drivers of 487 metabolic traits using LipocyteProfiler. 2021.07.17.452050

488 https://www.biorxiv.org/content/10.1101/2021.07.17.452050v1 (2021)

489 doi:10.1101/2021.07.17.452050.

490 29. Rajakumari, S. et al. EBF2 determines and maintains brown adipocyte identity. Cell $491 \quad$ Metab. 17, 562-574 (2013). 
492 30. Pulit, S. L. et al. Meta-analysis of genome-wide association studies for body fat

493 distribution in 694649 individuals of European ancestry. Hum. Mol. Genet. 28, 166-174

494 (2019).

495 31. Agrawal, S. et al. Inherited basis of visceral, abdominal subcutaneous and gluteofemoral

$496 \quad$ fat depots. 2021.08.24.21262564

497 https://www.medrxiv.org/content/10.1101/2021.08.24.21262564v1 (2021)

498 doi:10.1101/2021.08.24.21262564.

499 32. Willows, J. W. et al. Visualization and analysis of whole depot adipose tissue neural

$500 \quad$ innervation. iScience 24, 103127 (2021).

501 33. Sun, K., Halberg, N., Khan, M., Magalang, U. J. \& Scherer, P. E. Selective Inhibition of 502 Hypoxia-Inducible Factor $1 \alpha$ Ameliorates Adipose Tissue Dysfunction. Mol. Cell. Biol. 33, $503 \quad 904-917(2013)$

504 34. Roh, H. C. et al. Adipocytes fail to maintain cellular identity during obesity due to 505 reduced PPAR $\gamma$ activity and elevated TGF $\beta$-SMAD signaling. Mol. Metab. 42, 101086 $506 \quad(2020)$

507 35. Chiang, S.-H. et al. The protein kinase IKKepsilon regulates energy balance in obese $508 \quad$ mice. Cell 138, 961-975 (2009).

509 36. Park, J. et al. Progenitor-like characteristics in a subgroup of UCP1+ cells within white $510 \quad$ adipose tissue. Dev. Cell 56, 985-999.e4 (2021).

511 37. Chi, J. et al. Three-Dimensional Adipose Tissue Imaging Reveals Regional Variation in 512 Beige Fat Biogenesis and PRDM16-Dependent Sympathetic Neurite Density. Cell Metab. 27, 513 226-236.e3 (2018). 
514 38. Priest, C. \& Tontonoz, P. Inter-organ cross-talk in metabolic syndrome. Nat. Metab. 1, $515 \quad 1177-1188(2019)$.

516 39. Schling, P. \& Löffler, G. Cross talk between adipose tissue cells: impact on

517 pathophysiology. News Physiol. Sci. Int. J. Physiol. Prod. Jointly Int. Union Physiol. Sci. Am.

518 Physiol. Soc. 17, 99-104 (2002).

519 40. Kane, H. \& Lynch, L. Innate Immune Control of Adipose Tissue Homeostasis. Trends $520 \quad$ Immunol. 40, 857-872 (2019).

521 41. Efremova, M., Vento-Tormo, M., Teichmann, S. A. \& Vento-Tormo, R. CellPhoneDB: 522 inferring cell-cell communication from combined expression of multi-subunit ligand-receptor 523 complexes. Nat. Protoc. 15, 1484-1506 (2020).

524 42. Cao, Y. Angiogenesis and vascular functions in modulation of obesity, adipose 525 metabolism, and insulin sensitivity. Cell Metab. 18, 478-489 (2013).

526 43. Hubert, A. et al. Selective Deletion of Leptin Signaling in Endothelial Cells Enhances 527 Neointima Formation and Phenocopies the Vascular Effects of Diet-Induced Obesity in Mice. 528 Arterioscler. Thromb. Vasc. Biol. 37, 1683-1697 (2017).

529 44. Scott, R. A. et al. An Expanded Genome-Wide Association Study of Type 2 Diabetes in $530 \quad$ Europeans. Diabetes 66, 2888-2902 (2017).

531 45. Shungin, D. et al. New genetic loci link adipose and insulin biology to body fat 532 distribution. Nature 518, 187-196 (2015).

533 46. Huang, L. O. et al. Genome-wide discovery of genetic loci that uncouple excess adiposity 534 from its comorbidities. Nat. Metab. 3, 228-243 (2021).

535 47. Timshel, P. N., Thompson, J. J. \& Pers, T. H. Genetic mapping of etiologic brain cell 536 types for obesity. eLife $\mathbf{9}$, e55851 (2020). 
537 48. O’Rahilly, S. \& Farooqi, I. S. Human obesity as a heritable disorder of the central control

538 of energy balance | International Journal of Obesity.

539 https://www.nature.com/articles/ijo2008239.

540 49. Sailer, S., Keller, M. A., Werner, E. R. \& Watschinger, K. The Emerging Physiological

541 Role of AGMO 10 Years after Its Gene Identification. Life Basel Switz. 11, (2021).

542 50. Dupuis, J. et al. New genetic loci implicated in fasting glucose homeostasis and their

543 impact on type 2 diabetes risk. Nat. Genet. 42, 105-116 (2010).

544 51. Loh, N. Y. et al. RSPO3 impacts body fat distribution and regulates adipose cell biology

545 in vitro. Nat. Commun. 11, 2797 (2020). 


\section{FIGURES AND FIGURE LEGENDS}
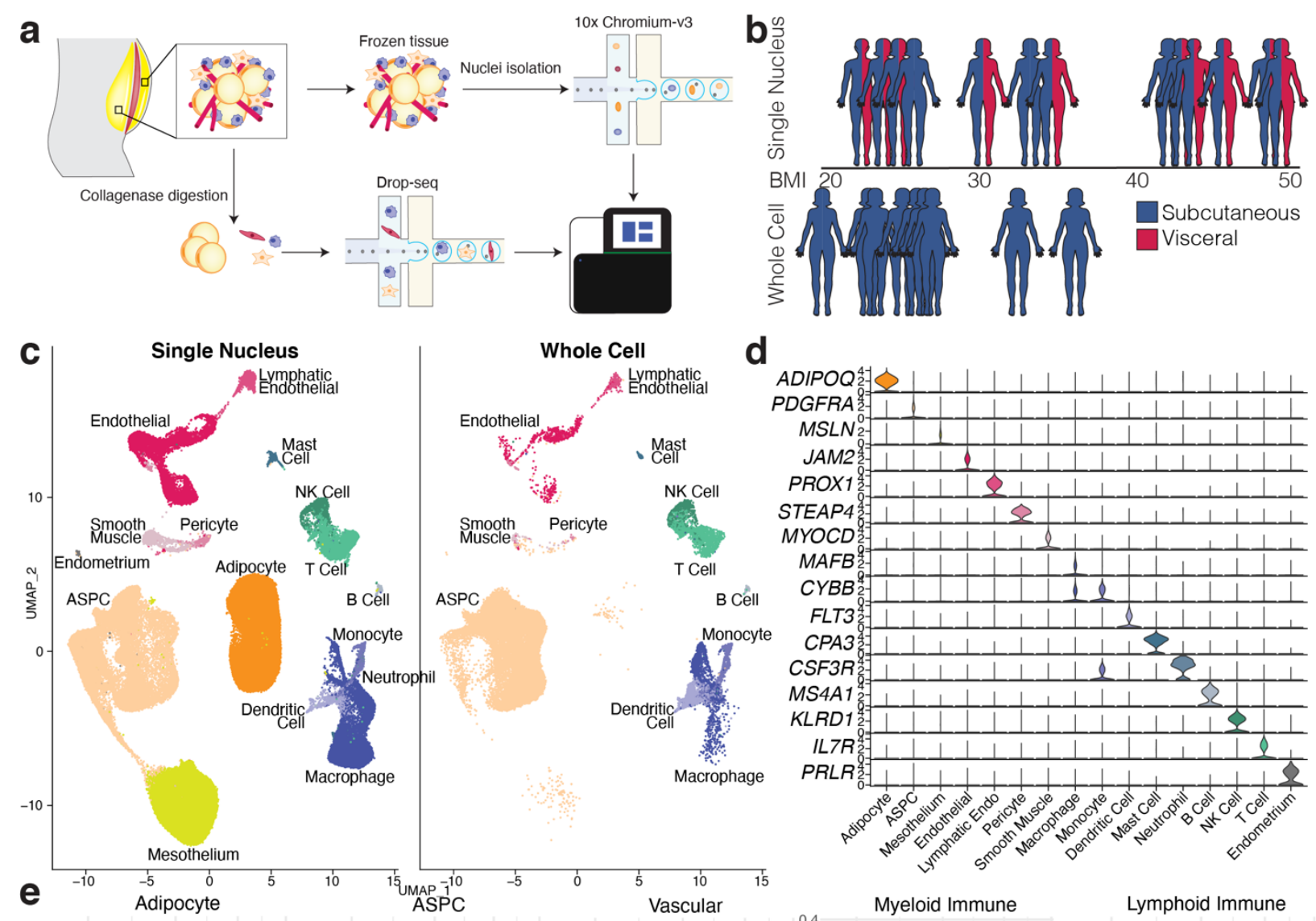

d
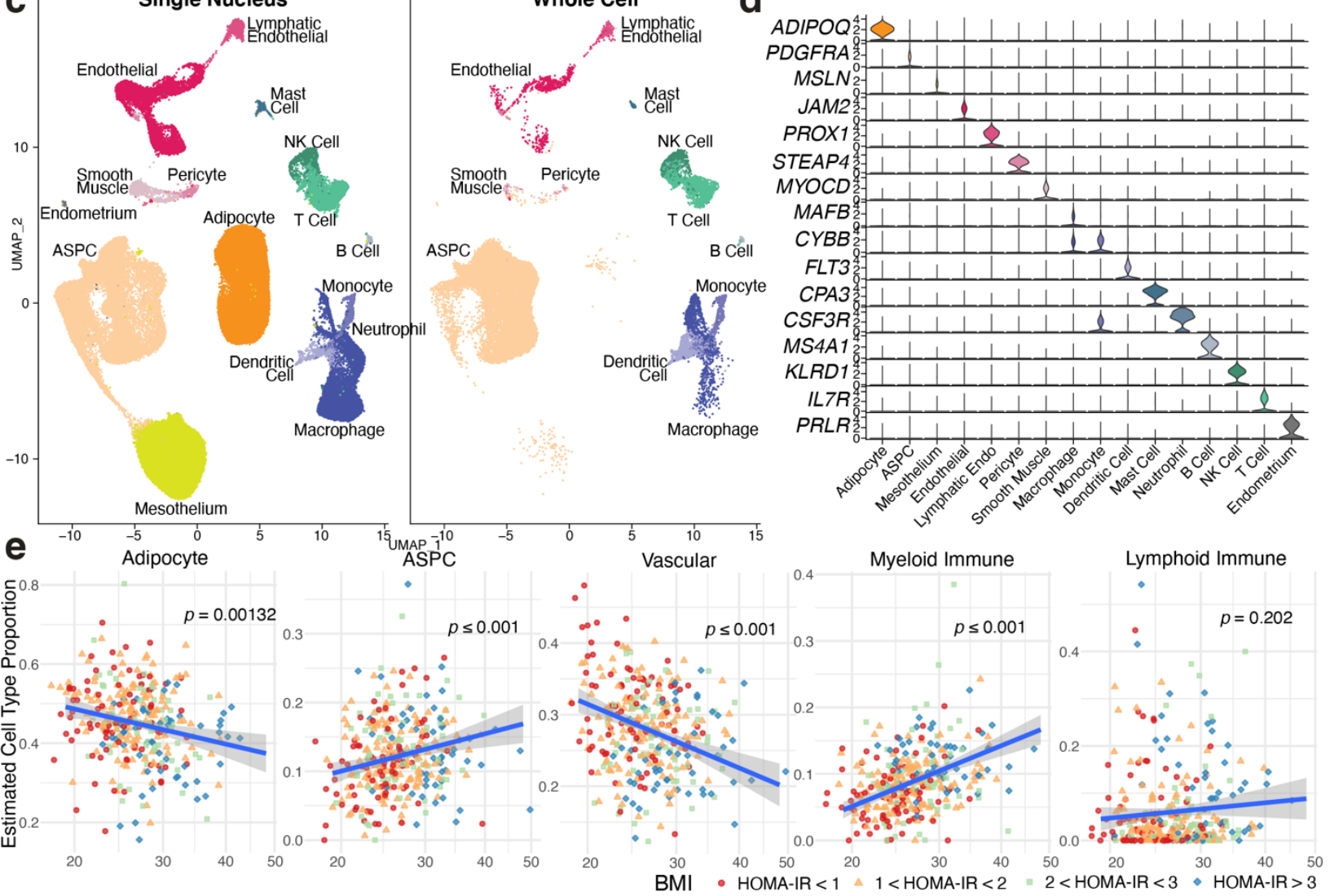

Fig. 1. A single cell atlas of human white adipose tissue. a, Schematic of workflows for scRNA-seq and sNuc-seq of human WAT. b, Graphical representation of the cohorts for both

548 studies. Only the sNuc-seq cohort contains VAT. c, UMAP projection of all 166,129 sequenced

549 human cells split by cohort. d, Marker genes for each cell population in the human WAT dataset.

550 e, Estimated cell type proportions in bulk RNA sequencing data of subcutaneous adipose tissue

551 from 331 individuals from the METSIM cohort calculated using sNuc-seq data as reference. 
552 Vascular cells include endothelial, lymphatic endothelial, pericytes, and smooth muscle cells.

553 Myeloid immune includes macrophages, monocytes, dendritic cells, mast cells and neutrophils,

554 and lymphoid immune includes B cells, NK cells, and T cells. For lines of best fit: Adipocytes $\mathrm{R}^{2}$

$555=0.031$, ASPCs $\mathrm{R}^{2}=0.034$, Vascular $\mathrm{R}^{2}=0.076$, Myeloid Immune $\mathrm{R}^{2}=0.13$, Lymphoid

$556 \quad$ Immune $\mathrm{R}^{2}=0.0049$. 

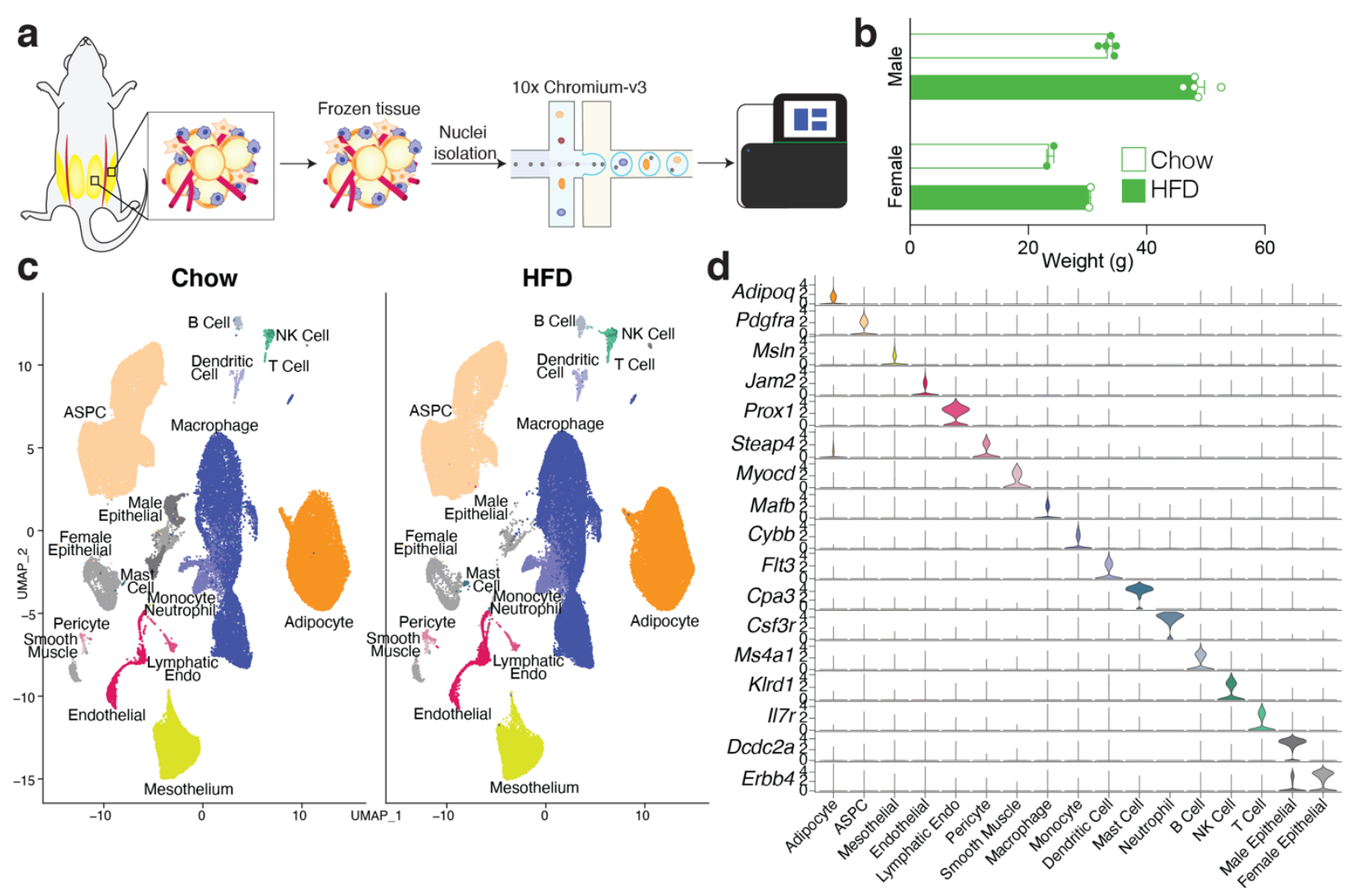

Fig. 2. A single cell atlas of mouse white adipose tissue. a, Schematic of workflow for sNucseq of mouse ING and EPI adipose tissue. b, Body weight of chow and high fat fed animals. c,

559 UMAP projection of all 197,721 sequenced mouse cells split by diet. d, Marker genes for each cell population in the mouse WAT dataset. 

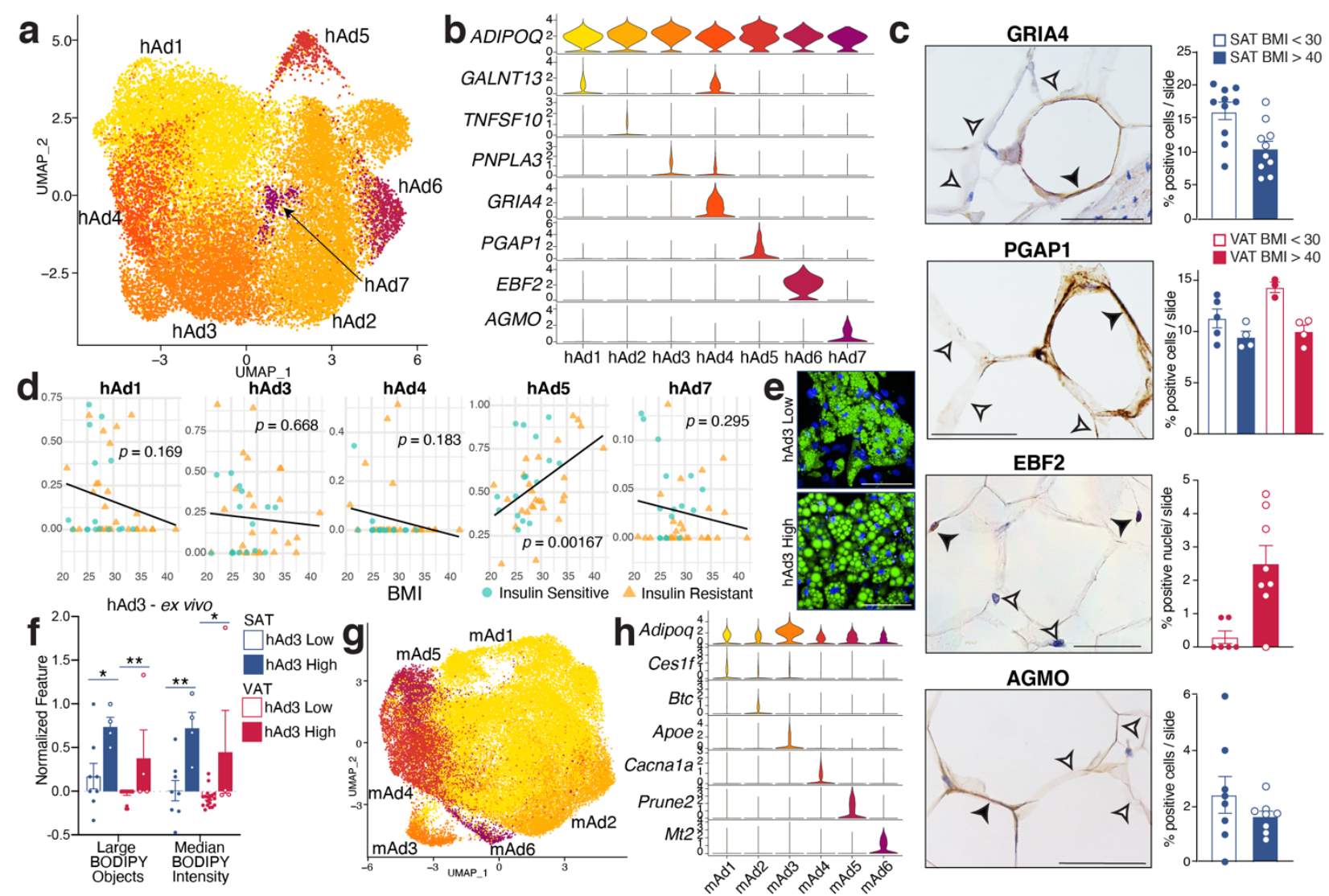

Fig. 3. Subclustering of human and mouse adipocytes reveals multiple distinct populations

that vary across depot and diet. a, UMAP projection of clusters formed by 25,871 human

563 white adipocytes. b, Expression of adipocyte marker $A D I P O Q$ as well as specific marker genes

564 for each adipocyte subpopulation. c, IHC for marker genes of adipocyte subpopulations hAd4,

565 hAd5, hAd6, and hAd7 in human adipose tissue and quantification of percentage of positive

566 adipocytes per slide in lean and obese individuals (GRIA4: 5 lean, 5 obese, 2 slides per person;

567 PGAP1: 5 lean SAT, 4 obese SAT, 3 lean VAT, 4 obese VAT, 1 slide per person; EBF2: 3 lean,

5684 obese, 2 slides per person; AGMO: 4 lean, 4 obese, 2 slides per person). Scale bars are $25 \mu \mathrm{m}$

569 for GRIA4, EBF2, and AGMO, $20 \mu \mathrm{m}$ for PGAP1. d, Estimated proportions of adipocyte

570 subpopulations in bulk RNA sequencing data of enzymatically isolated subcutaneous adipocytes

571 from 43 individuals plotted against subject BMI. e, Representative images of ex vivo 
572 differentiated human subcutaneous adipocytes predicted to have a low or high amount of hAd3

573 cells based on deconvolution of bulk RNA sequencing data. Green represents BODIPY staining,

574 blue represents Hoechst staining. Scale bars are $100 \mu \mathrm{m}$. f, Normalized count of BODIPY-

575 related features in human subcutaneous and visceral adipocytes differentiated ex vivo and

576 stratified into low and high hAd3-containing populations. g, UMAP projection of clusters formed

577 by 39,934 mouse white adipocytes. h, Expression of adipocyte marker Adipoq as well as specific

578 marker genes for each mouse adipocyte subpopulation. For bar graphs, error bars represent

579 standard error of the mean (SEM), ${ }^{*}, p<0.5, * *, p<0.1$. For lines of best fit: hAd1 $\mathrm{R}^{2}=0.046$,

$580 \mathrm{hAd} 3 \mathrm{R}^{2}=0.0045, \mathrm{hAd} 4 \mathrm{R}^{2}=0.043, \mathrm{hAd} 5 \mathrm{R}^{2}=0.22, \mathrm{hAd} 1 \mathrm{R}^{2}=0.027$ 


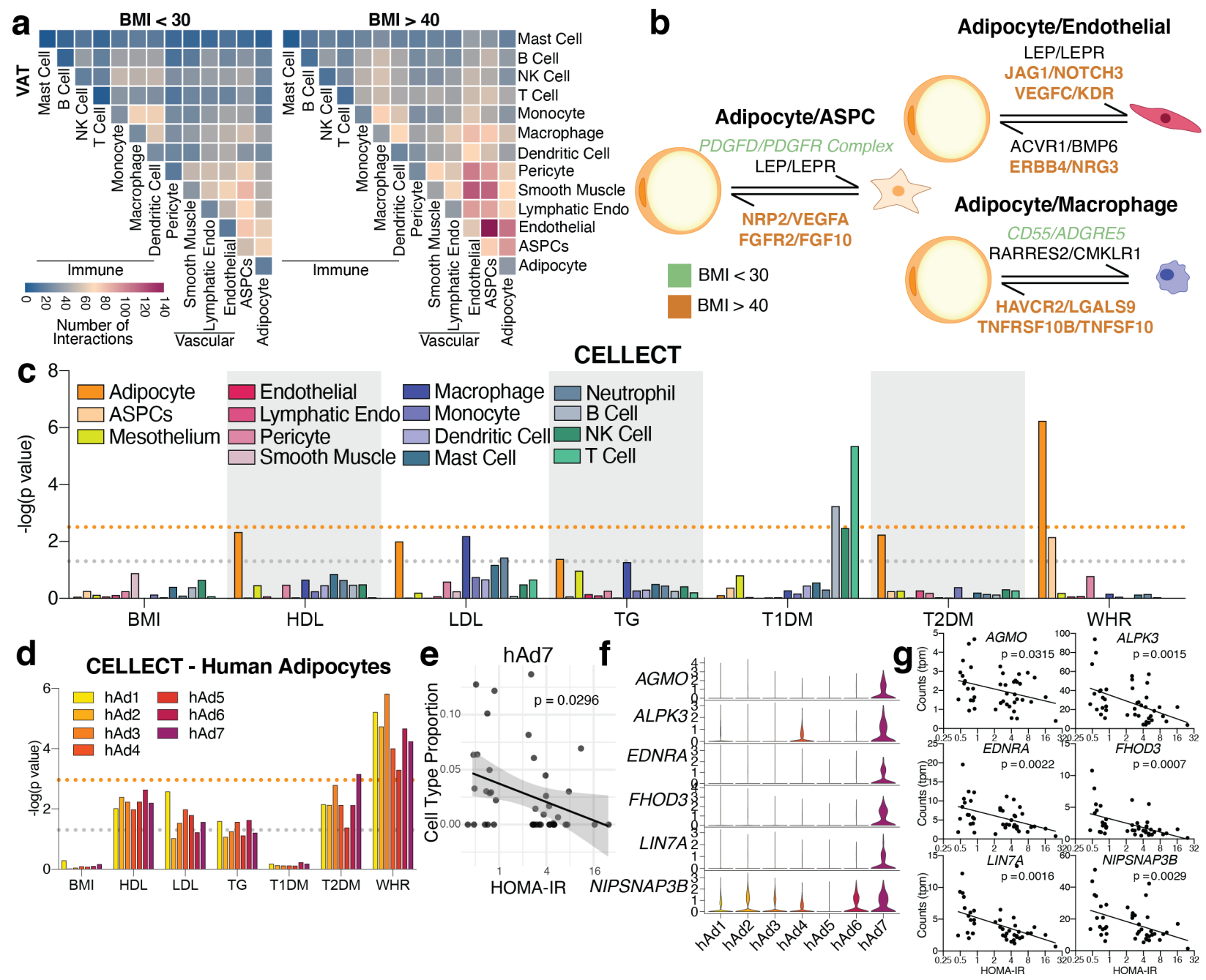

Fig. 4. Extensive cell-cell interactions in WAT and associations with human disease traits.

582 a, Heatmap showing number of significant interactions identified between cell types in VAT of

583 low $(<30)$ and high $(>40)$ BMI individuals as determined by CellphoneDB. b, Selected

584 interactions between adipocytes and ASPCs, endothelial cells, and macrophages identified using

585 CellphoneDB; orange and green indicate interactions that are significant only in BMI $>40$ or

586 only in BMI $>30$, respectively. c, CELLECT $p$ values of the association between cell types in the

587 human adipose sNuc-seq dataset with GWAS studies. The grey line represents $p=0.05$ and the

588 orange line represents significant $\mathrm{p}$ value after Bonferroni adjustment $(p=0.003)$, based on

589 number of cell types queried. Both T2D and WHR were BMI-adjusted. d, CELLECT $p$ values 
590 for adipocyte subpopulations. The grey line represents $p=0.05$ and the orange line represents

591 significant $p$ value after Bonferroni adjustment $(p=0.001)$, based on all cell subtypes queried. e,

592 Estimated cell type proportion of hAd7 in bulk RNA-seq data of enzymatically isolated

593 subcutaneous adipocytes from 43 individuals plotted against HOMA-IR. For line of best fit, $\mathrm{R}^{2}=$

594 0.11. f-g, Expression of hAd7 marker genes negatively correlated with HOMA-IR in human

595 adipocyte subpopulations (f) and bulk RNA sequencing data of human adipocytes (g).

\section{METHODS}

\section{Collection of human adipose tissue samples.}

599 Drop-Seq and Floated adipocyte bulk RNA-seq

600 Subcutaneous adipose tissue was collected under Beth Israel Deaconess Medical Center

601 Committee on Clinical Investigations IRB 2011P000079. Potential subjects were recruited in a

602 consecutive fashion, as scheduling permitted, from the plastic surgery operating room rosters at

603 Beth Israel Deaconess Medical Center. Male and female subjects over the age of 18 undergoing

604 elective plastic surgery procedures and free of other acute medical conditions were included and

605 provided written informed consent preoperatively. Excess adipose tissue from the surgical site

606 was collected at the discretion of the surgeon during the normal course of the procedure. Subjects

607 with a diagnosis of diabetes, or taking insulin-sensitizing medications such as thiazolidinediones

608 or metformin, chromatin-modifying enzymes such as valproic acid, anti-retroviral medications,

609 or drugs known to induce insulin resistance such as mTOR inhibitors or systemic steroid

610 medications, were excluded.

612 sNuc-Seq 
613 Subcutaneous and visceral adipose tissue was collected under BIDMC Committee on Clinical

614 Investigations IRB 2011P000079 and University of Pittsburgh Medical Center STUDY

615 19010309. At BIDMC, potential subjects were recruited in a consecutive fashion, as scheduling

616 permitted, from the gynecological, vascular, and general surgery rosters. Male and female

617 subjects over the age of 18 undergoing plastic surgery (panniculectomy, thighplasty or deep

618 inferior epigastric perforators), gynecological surgery (total abdominal hysterectomy and

619 bilateral salpingo-oophorectomy) or general surgery (cholecystectomy (CCY) or colin polyp

620 surgery) and free of other acute medical conditions were included and provided written informed

621 consent preoperatively. Excess adipose tissue from the surgical site was collected at the

622 discretion of the surgeon during the normal course of the procedure. The exclusion criteria were

623 any subjects taking thiazolidinediones, chromatin-modifying enzymes such as valproic acid, anti-

624 retroviral medications, and drugs known to induce insulin resistance such as mTOR inhibitors or

625 systemic steroid medications. At UPMC, inclusion criteria were patients receiving bariatric

626 surgery (Vertical Sleeve Gastrectomy or Roux en Y Gastric Bypass) or lean controls (hernia or

627 CCY surgeries) ages 21-60, exclusion criteria were diagnosis of diabetes (Type 1 or Type 2),

628 pregnancy, alcohol or drug addiction, bleeding or clotting abnormality, or inflammatory

629 abdominal disease. All patients provided written informed consent preoperatively. Excess

630 adipose tissue from the surgical site was collected at the discretion of the surgeon during the

631 normal course of the procedure. $200-500 \mathrm{mg}$ samples were flash frozen immediately after

632 collection for downstream processing.

633

\section{Mouse adipose tissue samples}


635 All animal experiments were performed under a protocol approved by the BIDMC Institutional

636 Animal Care and Use Committee. Male C57B1/6J 16-week-old high fat diet fed (JAX 380050)

637 and chow fed (JAX 380056) mice were obtained from The Jackson Laboratory and maintained

638 on $60 \%$ high fat diet (Research Diets, D12492) or chow diet (8664 Harlan Teklad, 6.4\% wt/wt

639 fat), respectively, for three weeks before sacrifice. Female 6-week-old chow fed C57B1/6J mice

640 (JAX 380056) were maintained on 60\% high fat diet for 13 weeks before sacrifice. Mice were

641 maintained under a $12 \mathrm{hr}$ light/12hr dark cycle at constant temperature $\left(23^{\circ} \mathrm{C}\right)$ with free access to

642 food and water.

\section{Mature human adipocyte sample preparation}

645 Purification of mature human adipocytes.

646 Whole tissue subcutaneous adipose specimens were freshly collected from the operating room.

647 Skin was removed, and adipose tissue was cut into 1- to 2-inch pieces and rinsed thoroughly with

$64837^{\circ} \mathrm{C}$ PBS to remove blood. Cleaned adipose tissue pieces were quickly minced in an electric

649 grinder with 3/16-inch hole plate, and $400 \mathrm{ml}$ of sample was placed in a 2-1 wide-mouthed

650 Erlenmeyer culture flask with $100 \mathrm{ml}$ of freshly prepared blendzyme (Roche Liberase TM,

651 research grade, cat. no. 05401127001 , in PBS, at a ratio of $6.25 \mathrm{mg}$ per $50 \mathrm{ml}$ ) and shaken in a 37

$652{ }^{\circ} \mathrm{C}$ shaking incubator at 120 r.p.m. for $15-20$ min to digest until the sample appeared uniform.

653 Digestion was stopped with $100 \mathrm{ml}$ of freshly made KRB (5.5 mM glucose, $137 \mathrm{mM} \mathrm{NaCl}, 15$

$654 \mathrm{mM}$ HEPES, $5 \mathrm{mM} \mathrm{KCl,} 1.25 \mathrm{mM} \mathrm{CaCl}$, $0.44 \mathrm{mM} \mathrm{KH} 2 \mathrm{PO} 4,0.34 \mathrm{mM} \mathrm{Na} 2 \mathrm{HPO} 4$ and $0.8 \mathrm{mM}$

$655 \mathrm{MgSO} 4)$, supplemented with 2\% BSA. Digested tissue was filtered through a $300 \mu \mathrm{M}$ sieve and

656 washed with KRB/albumin and flow through until only connective tissue remained. Samples

657 were centrifuged at $233 \mathrm{~g}$ for $5 \mathrm{~min}$ at room temperature, clear lipid was later removed, and 
658 floated adipocyte supernatant was collected, divided into aliquots and flash-frozen in liquid

659 nitrogen.

660

661 Sample selection and Bulk-RNA-seq library construction

662 Fasting serum was collected and insulin, glucose, free fatty acids, and a lipid panel were

663 measured by Labcorp. BMI measures were derived from electronic medical records and

664 confirmed by self-reporting, and measures of insulin resistance, the homeostasis model

665 assessment-estimated insulin resistance index (HOMA-IR) and revised quantitative insulin

666 sensitivity check index (QUICKI) were calculated ${ }^{52,53}$. Female subjects in the first and fourth

667 quartiles for either HOMA-IR or QUICKI and matched for age and BMI were processed for

668 RNA-seq.

670 Total RNA from $\sim 400 \mu$ of thawed floated adipocytes was isolated in TRIzol reagent

671 (Invitrogen) according to the manufacturer's instructions. For RNA-seq library construction,

672 mRNA was purified from 100 ng of total RNA by using a Ribo-Zero rRNA removal kit

673 (Epicentre) to deplete ribosomal RNA and convert into double-stranded complementary DNA by

674 using an NEBNext mRNA Second Strand Synthesis Module (E6111L). cDNA was subsequently

675 tagmented and amplified for 12 cycles by using a Nextera XT DNA Library Preparation Kit

676 (Illumina FC-131). Sequencing libraries were analyzed with Qubit and Agilent Bioanalyzer,

677 pooled at a final loading concentration of $1.8 \mathrm{pM}$ and sequenced on a NextSeq500.

678

679 Single Cell and Single Nucleus sample preparation and processing

680 SVF isolation and Drop-seq. 
681 Adipose tissue samples were collected and processed as above. After removal of floated

682 adipocytes, remaining supernatant was aspirated and the remaining pelleted stromal vascular

683 fraction (SVF)was combined from multiple tubes. The combined SVF was washed 2 times with

$68450 \mathrm{ml}$ cold PBS with 233g for 5 min centrifugation between washes. Erythrocytes were depleted

685 with two rounds of $25 \mathrm{ml}$. ACK lysing buffer (Gibco ${ }^{\mathrm{TM}}$ A1049201) exposure (5 minutes at RT

686 followed by $233 \mathrm{~g}$ x 5 min centrifugation). Remaining SVF pellet was further washed x 2 with

$68750 \mathrm{ml}$ cold PBS prior to counting on hematocytometer and loading onto Drop-seq microfluidic

688 devices. Drop-seq was performed as described ${ }^{54}$, with the following modifications: first, flow

689 rates of $2.1 \mathrm{~mL} / \mathrm{h}$ were used for each aqueous suspension and $12 \mathrm{~mL} / \mathrm{h}$ for the oil. Second,

690 libraries were sequenced on the Illumina NextSeq500, using between 1.6-1.7 pM in a volume of

$691 \quad 1.2 \mathrm{~mL} \mathrm{HT1}$ and $3 \mathrm{~mL}$ of $0.3 \mu \mathrm{M}$ Read1CustSeqB

692 (GCCTGTCCGCGGAAGCAGTGGTATCAACGCAGAGTAC) using 20 × 8 x $60 \mathrm{read}$

693 structure.

$695 s N u c-S e q$

696 Nuclei were isolated from frozen mouse and human adipose tissue samples for 10x snRNA-seq

697 using a slightly modified approach to what was previously described ${ }^{55-57}$. Samples were kept

698 frozen on dry ice until immediately before nuclei isolation, and all sample handling steps were

699 performed on ice. Each flash-frozen adipose tissue sample was placed into a gentleMACS C tube

700 (Miltenyi Biotec) with $2 \mathrm{~mL}$ freshly prepared TST buffer (0.03\% Tween 20 [Bio-Rad], 0.01\%

701 Molecular Grade BSA [New England Biolabs], $146 \mathrm{mM} \mathrm{NaCl}$ [ThermoFisher Scientific], $1 \mathrm{mM}$

$702 \mathrm{CaCl} 2$ [VWR International], $21 \mathrm{mM} \mathrm{MgCl2}$ [Sigma Aldrich], and $10 \mathrm{mM}$ Tris-Hcl pH 7.5

703 [ThermoFisher Scientific] in Ultrapure water [ThermoFisher Scientific]) with or without 0.2 U/ 
$\mu \mathrm{L}$ of Protector RNase Inhibitor (Sigma Aldrich). gentleMACS C tubes were then placed on the gentleMACS Dissociator (Miltenyi Biotec) and tissue was dissociated by running the program "mr_adipose_01" twice, and then incubated on ice for 10 minutes. Lysate was passed through a $40 \mu \mathrm{m}$ nylon filter (CellTreat) and collected into a $50 \mathrm{~mL}$ conical tube (Corning). Filter was rinsed with $3 \mathrm{~mL}$ of freshly prepared ST buffer buffer $(146 \mathrm{mM} \mathrm{NaCl}, 1 \mathrm{mM} \mathrm{CaCl} 2,21 \mathrm{mM}$ $\mathrm{MgCl}$; $10 \mathrm{mM}$ Tris-Hcl $\mathrm{pH} 7.5$ ) with or without $0.2 \mathrm{U} / \mu \mathrm{L}$ RNase Inhibitor, and collected into the same tube. Flow-through was centrifuged at $500 \mathrm{x}$ g for 5 minutes at $4^{\circ} \mathrm{C}$ with brake set to

711 low. Following centrifugation, supernatant was removed, and the nuclear pellet was resuspended in 50 - $200 \mu$ PBS pH 7.4 (ThermoFisher Scientific) with $0.02 \%$ BSA, with or without $0.2 \mathrm{U} / \mu \mathrm{L}$ RNase Inhibitor. In order to reduce ambient mRNA, the nuclear pellets of some samples were

714 washed 1-3 times with $5 \mathrm{~mL}$ of PBS-0.02\% BSA before final resuspension. An aliquot of nuclei

715 from each sample was stained with NucBlue (Thermofisher Scientific), counted in a

716 hemocytometer using fluorescence to identify intact nuclei, and then immediately loaded on the

717 10x Chromium controller (10x Genomics) according to the manufacturer's protocol.

718 For each sample, 10,000-16,500 nuclei were loaded in one channel of a Chromium Chip (10x

719 Genomics). The Single Cell 3'v3.1 chemistry was used to process all samples. cDNA and gene 720 expression libraries were generated according to the manufacturer's instructions (10x Genomics).

721 cDNA and gene expression library fragment sizes were assessed with a DNA High Sensitivity

722 Bioanalyzer Chip (Agilent). cDNA and gene expression libraries were quantified using the Qubit

723 dsDNA High Sensitivity assay kit (ThermoFisher Scientific). Gene expression libraries were

724 multiplexed and sequenced on the Nextseq 500 (Illumina) with a 75-cycle kit and the following

725 read structure: Read 1: 28 cycles, Read 2: 55 cycles, Index Read 1: 8 cycles. 


\section{Sequencing, read alignments, and quality control}

728 Single-cell/nucleus RNA-seq data analysis.

729 Raw sequencing reads were demultiplexed to FASTQ format files using bcl2fastq (Illumina;

730 version 2.20.0). Digital expression matrices were generated from the FASTQ files using the

731 Drop-Seq tools (https://github.com/broadinstitute/Drop-seq) pipeline, with appropriate

732 adjustments made to the default program parameters to account for the different read-structures

733 in the scRNA Drop-Seq data and sNuc 10X data. Reads from mouse and human were aligned

734 with $\mathrm{STAR}^{58}$ (version 2.7.3) against the GRCm38 and GRCh38 genome assemblies,

735 respectively. Gene counts were obtained, per-droplet, by summarizing the unique read

736 alignments across exons and introns in appropriate GENCODE annotations (release 16 of the

737 mouse annotation and release 27 of the human annotation). In order to adjust for downstream

738 effects of ambient RNA expression within mouse nuclei (hereafter “cells"), we used

739 CellBender ${ }^{59}$ (version 0.2.0) to remove counts due to ambient RNA molecules from the count

740 matrices and to estimate the true cells. We also used CellBender to distinguish droplets

741 containing cells from droplets containing only ambient RNA, by selecting droplets with $>50 \%$

742 posterior probability of containing a cell. We compared the true cell estimation obtained using

743 CellBender against the same using the DropletUtils software package ${ }^{60}$, which estimates ambient

744 RNA expression levels but does not remove any ambient counts, keeping only the cells that were

745 marked as not ambient by both algorithms. To address ambient RNA in the human sNuc data, we

746 calculated spliced and unspliced RNA content in each cell, because nuclei have a high unspliced

747 RNA content, a high percentage of spliced RNA indicates a high ambient RNA content. We

748 therefore removed sNuc-seq cells containing over $75 \%$ spliced RNA. All samples were assessed

749 for doublet content using scrublet ${ }^{61}$ version 0.2 .1 , and cells called as doublets were removed 
750 before further analysis. All cells were further filtered to have greater than 400 UMIs with $<10 \%$

751 of UMIs from mitochondrial genes. Genes were filtered such that only genes detected in two or

752 more cells were retained. For the human data, the median number of UMIs detected per cell was

7532559 and the median number of genes detected per cell was 1524 . For the mouse data, the

754 median number of UMIs detected per cell was 2291 and the median number of genes detected

755 per cell was 1369.

757 Bulk RNA-seq Analysis.

758 Raw sequencing reads were demultiplexed by using bcl2fastq (Illumina). Salmon ${ }^{62}$ (version

759 1.1.0) was used to simultaneously map and quantify transcript abundances of hg19 genes

760 annotated by release 19 of the GENCODE project's human reference. Salmon was run using

761 "full" selective alignment (SAF) with mapping validation as described previously ${ }^{63}$. Gene counts

762 were summarized from transcript abundances using the "tximport" package for $\mathrm{R}^{64}$.

763

\section{Integration, clustering, subclustering, and annotation}

765 Integration, clustering and subclustering analysis were performed using Seurat 3.9.965. The gene

766 counts were normalized using SCTransform ${ }^{66}$, and regressed on mitochondrial read percentage,

767 ribosomal read percentage, and cell cycle score as determined by Seurat. In order to avoid

768 smoothing over depot differences, for integration human and mouse data were grouped by

769 'individual', i.e., if both subcutaneous and visceral adipose tissue for an individual human or

770 mouse were available, they were pooled together during this step. Individuals were integrated

771 with reciprocal PCA, using individuals that had both subcutaneous and visceral samples as

772 references. As a result, the human and mouse references were comprised exclusively from the 
773 sNuc seq cohort. To integrate, references were integrated together, then the remaining samples-

774 sNuc seq individuals with only subcutaneous data as well as all Drop-seq samples — were

775 mapped to the reference. For clustering, 5000 variable genes were used, and ribosomal and

776 mitochondrial genes were removed from the variable gene set before running PCA and

777 calculating clusters using a Louvain algorithm, $40 \mathrm{PCs}$, and a resolution of 0.5 . Clusters were

778 identified as adipocytes, preadipocytes, mesothelial cells, vascular cells, or immune cells using

779 marker genes, subset into individual objects, and re-integrated using the above method. Samples

780 with fewer than 50 cells in the subset were removed before re-integration. This led to samples

781 having artificially fewer cells in some instances_-for example some Drop-seq samples had cells

782 that clustered with adipocytes, but these cells were removed in subclustering because the small

783 numbers of cells introduced too much variability into the integration. Subclustering was

784 performed using a range of variable genes (1000-2000), PCs (10-40) and resolutions (0.2-0.6).

785 Markers were calculated using a non-parametric Wilcoxon rank sum test and clusters were

786 evaluated based on the distinctness of called markers to determine the final subclustering

787 conditions. In the subclustered objects, we removed clusters that appeared to represent doublets

788 based on the score assigned by scrublet ${ }^{61}$, or that appeared to be driven by high ambient RNA

789 content as determined by levels of mitochondrial genes and spliced/unspliced RNA ratio. The

790 remaining clusters were annotated based on marker gene expression. In some cases, smaller

791 subclusters (T and NK cells, B cells, monocytes/neutrophils) were further subset and PCA and

792 clustering analysis but not integration was re-run in order to assign clusters. After subcluster

793 annotation, identities were mapped back onto the original object and cells that were removed

794 from the subclustered objects were similarly removed from the all-cell object. 


\section{Deconvolution of bulk RNA-seq data}

797 Bulk RNA sequencing data for subcutaneous adipose tissue from the METSIM cohort were

798 obtained as described previously ${ }^{13}$. Only individuals with available metabolic phenotyping data

799 were used for the deconvolution analysis. Bulk RNA sequencing data for floated human

800 adipocytes were obtained described above. Deconvolution analysis was performed using

$801 \mathrm{MuSiC}^{12}$ (version 0.1.1) with human sNuc subcutaneous all cell or adipocyte data as reference.

802 Marker genes used for deconvolution can be found in Supplemental Table 1.

Comparison between mouse and human datasets

805 Mapping of mouse cells onto human clusters was performed using Seurat multimodal reference

806 mapping ${ }^{67}$. To run, for the all-cell and each subset, the mouse data was prepared by extracting

807 the counts matrix from the mouse sNuc object and mapping the mouse gene names to their

808 human orthologs using a database of ortholog mappings from Mouse Genome Informatics

809 (http://www.informatics.jax.org/homology.shtml). In the case of multi-mapping, the first

810 ortholog pair was used. The mouse object was then split by sample and mapped onto the sNuc-

811 seq data from the matching human all-cell or subset object using the RNA assay and PCA

812 reduction.

\section{Immunohistochemistry}

815 Subcutaneous (abdominal) and omental adipose tissue biopsies belonging to lean and obese 816 women (GRIA4: subcutaneous, 5 lean and 5 obese individuals; PGAP1: subcutaneous, 5 lean, 4 817 obese, visceral 3 lean, 4 obese; EBF2: omental, 3 lean and 4 obese individuals; AGMO: 818 subcutaneous, 4 lean and 4 obese individuals, for all experiments two slides per individual for 
819 GRIA4, EBF2, AGMO, one slide per individual for PGAP1) were fixed (overnight in 4\%

820 paraformaldehyde at $4^{\circ} \mathrm{C}$, dehydrated, paraffin embedded and sectioned ( $4 \mu \mathrm{m}$ thick). The

821 following primary antibodies and respective dilution were used: GRIA4, 1:200, Cat \#23350-1-AP,

822 Proteintech; PGAP1, 1:400, Cat. \#55392-1-AP, Proteintech EBF2, 1:1000, Cat. \#AF7006, R\&D

823 systems; AGMO (TMEM195) 1:100, Cat \#orb395684, Biorbyt. In brief, after rinsing in PBS,

824 tissue slices were blocked with 3\% normal goat serum and incubated with the primary antibody in

825 PBS, overnight at $4^{\circ} \mathrm{C}$. After a thorough rinse in PBS, sections were incubated in 1:200 v/v

826 biotinylated secondary antibody solution for 30 minutes (Invitrogen), rinsed in PBS and incubated

827 in avidin-biotin-peroxidase complex (ABC Standard, Vector Laboratories), washed several times

828 in PBS and lastly incubated in 3,3'-diaminobenzidine tetrahydrochloride $(0.05 \%$ in $0.05 \mathrm{M}$ Tris

829 with $\left.0.03 \% \mathrm{H}_{2} \mathrm{O}_{2} ; 5 \mathrm{~min}\right)$. After immunohistochemical staining, sections were counterstained with

830 hematoxylin, dehydrated in ethanol, cleared in xylene and covered with coverslip using Eukitt

831 (Merck). All observations were performed using Nikon Eclipse E800 light microscope.

833 Immunofluorescence microscopy of mature human adipocytes

834 Adipocyte immunofluorescence protocol was adapted from Sárvári et al ${ }^{9}$. Abdominal

835 subcutaneous adipose tissue was collected from two adult female human subjects (BMI 24.9 and

83640.3 ) as above and placed on ice. Tissue was minced and digested with $1 \mathrm{mg} / \mathrm{mL}$ type II

837 collagenase (Sigma-Aldrich, C6885) in Hanks' balanced salt solution supplemented with 0.5\%

838 fatty acid-free BSA (Sigma-Aldrich, A6003) at $37^{\circ}$ in a water bath with constant shaking at 250

$839 \mathrm{rpm}$. The cell suspension was filtered through a $250 \mu \mathrm{M}$ nylon mesh strainer (Thermo, 87791)

840 and washed three times with Krebs-Ringer bicarbonate buffer containing $1 \%$ fatty acid-free

841 BSA. All washes throughout this protocol were performed without centrifugation to minimize 
842 adipocyte damage and loss; cell suspension was maintained upright for at least 5 minutes to

843 allow mature adipocytes to float, and infranatant was removed with a needle and syringe. The

844 floating adipocytes were fixed with 2\% PFA and 1\% sucrose in PBS for 30 minutes with

845 constant rotation followed by three washes with $2 \%$ fatty acid-free BSA in PBS. Adipocytes

846 were subsequently permeabilized with $0.5 \%$ Triton-X (Thermo, 28314) in PBS for five minutes,

847 and incubated with $2.5 \mu \mathrm{g} / \mathrm{mL}$ trypsin (Corning, 25053CI) in PBS for 10 minutes at $37^{\circ}$ in a

848 water bath with constant shaking. Adipocytes were then blocked with $2 \%$ fatty acid-free BSA in

849 PBS for 30 minutes, and incubated overnight at room temperature with rabbit polyclonal anti-

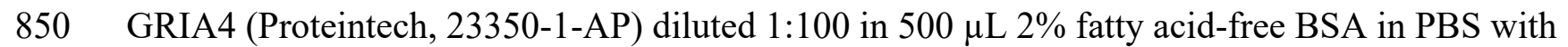

851 constant rotation. The adipocytes were then washed twice for 10 minutes each with $0.1 \%$ fatty

852 acid-free BSA and 0.05\% Tween-20 (Sigma-Aldrich, P9416) in PBS, followed by incubation

853 with goat anti-rabbit Alexa Fluor 546 (Thermo, A-11035) secondary antibody diluted 1:500 in

$8542 \%$ fatty acid-free BSA for 2 hours with rotation. For the final 30 minutes of incubation, Hoechst

85533342 (Thermo, 62249) and BODIPY 493/503 (Invitrogen, D3922) were added at 1:500

856 dilutions. Adipocytes were washed twice and resuspended in $300 \mu \mathrm{L}$ Fluoromount G (Southern

857 Biotech, 0100-01) and mounted on glass slides with 1.4-1.6 mm concavity wells (Electron

858 Microscopy Sciences, 71878-03). A sample of adipocytes was also incubated as above but

859 without primary antibody to verify the specificity of the secondary antibody. Fluorescence

860 images were acquired using Zeiss LSM 880 Upright Laser Scanning Confocal Microscope with

861 filter cubes for DAPI, GFP, and Rhodamine in parallel using the 20X objective and processed

862 using Zen Black 2.3 software. Images were analyzed and counted with ImageJ v. 1.53k. 


\section{Ex vivo differentiation and transcriptional and high-content image-based characterization} of differentiating primary human adipocyte progenitors

866 We obtained adipocyte progenitors from subcutaneous and visceral adipose tissue from patients

867 undergoing a range of abdominal laparoscopic surgeries (sleeve gastrectomy, fundoplication or

868 appendectomy). The visceral adipose tissue is derived from the proximity of the angle of His and

869 subcutaneous adipose tissue obtained from beneath the skin at the site of surgical incision.

870 Additionally, human liposuction material was obtained. Each participant gave written informed

871 consent before inclusion and the study protocol was approved by the ethics committee of the

872 Technical University of Munich (Study № 5716/13). Isolation of AMSCs was performed as

873 previously described ${ }^{28}$, and cells were differentiated in culture over 14 days. Ex vivo

874 differentiated adipocytes were stained and imaged, and features were extracted using

875 LipocyteProfiler as described in Laber et al. RNA-sequencing libraries were prepared and

876 sequenced and QC'ed as previously described ${ }^{28}$. Bulk-RNA sequencing counts from

877 subcutaneous and visceral samples differentiated for 14 days were deconvoluted using both

878 subcutaneous and visceral adipocytes as reference as described above. Raw images collected

879 during LipocyteProfiler analysis were randomly selected from samples predicted to have high or

880 low content of hAd3, hAd5, or hAd6 adipocytes, and pseudocolored and combined using Adobe

881 Photoshop.

\section{Gene Pathway Analysis}

884 Analysis of enriched pathways in adipocyte markers was performed using clusterProfiler ${ }^{68}$

885 (version 3.16.1). Adipocyte cluster markers were filtered to an adjusted $p$-value $<.05$, then 
evaluated for enrichment in GO biological pathways or KEGG pathways containing under 300 genes.

\section{Identification and analysis of EBF2 SNP association with visceral adiposity}

VAT, ASAT, and GFAT volumes in 40,032 individuals from the UK Biobank ${ }^{69,70}$ who underwent MRI imaging were quantified as described elsewhere ${ }^{71}$. Variant rs4872393 was identified as a lead SNP associated with VATadjBMI and waist-to-hip ratio from summary statistics of two prior studies ${ }^{31,72}$. Among the cohort who underwent MRI imaging, all variants at this locus ( $\pm 250 \mathrm{~kb}$ around rs4872393) with MAF $>=0.005$ and imputation quality (INFO) score $>=0.3$ were analyzed. For all 554 nominally significant $(\mathrm{P}<0.05)$ variants associated with VATadjBMI in this region, a secondary conditional analysis testing for association with VATadjBMI was performed controlling for rs4872393 carrier status $\left(\mathrm{P}<0.05 / 554=9 \times 10^{-5}\right)$.

898 Participants were excluded from analysis if they met any of the following criteria: (1) mismatch between self-reported sex and sex chromosome count, (2) sex chromosome aneuploidy, (3)

900 genotyping call rate $<0.95$, or (4) were outliers for heterozygosity. Up to 37,641 participants

901 were available for analysis. Fat depot volumes adjusted for BMI and height (“adj” traits) were

902 calculated by taking the residuals of the fat depot in sex-specific linear regressions against age at 903 the time of MRI, age squared, BMI, and height ${ }^{31}$. Each trait was scaled to mean 0 and variance 1 904 in sex-specific groups before being combined for analysis. Linear regressions between a given

905 trait-variant pair were adjusted for age at the time of imaging, age squared, sex, the first 10 906 principal components of genetic ancestry, genotyping array, and MRI imaging center. Analyses

907 were performed using R 3.6.0 (R Project for Statistical Computing). EBF2 regional visualization 908 plot was made with the LocusZoom online tool ${ }^{73}$. 
Calculation of pseudobulk datasets to estimate adipose innervation

911 Approximate bulk RNA-seq datasets (pseudobulk) were obtained for visceral sNuc-seq samples

912 by summing the total expression per-gene across all droplets containing a valid 10X cell barcode.

913 This includes all cells that would normally have been removed in the single-nuclei studies by any

914 of the filtering criteria (above): doublet score, splicing content, droplets with fewer than 400

915 UMIs, etc, in order to preserve the ambient RNA present in otherwise empty droplets. Repeated

916 UMIs were still collapsed into single counts (per-droplet) before summing. Levels of pan-

917 neuronal markers were calculated using this pesudobulk dataset and plotted against the

918 proportion of visceral populations hAd2 and hAd6 relative to total adipocytes in each sample.

\section{Prediction of cell-cell interactions}

921 Analysis of cell-cell interactions was performed using CellphoneDB ${ }^{41}$ (version 2.0.0). For human

922 data, sNuc-seq counts data was split into files containing cells from subcutaneous and visceral fat

923 from individuals with BMI lower than 30 or higher than 40. CellphoneDB with statistical

924 analysis was run on each file separately to evaluate interactions in each condition. For mouse

925 data, counts data was split into files containing cells from the inguinal and perigonadal fat of

926 chow and high fat diet fed mice. Mouse gene names were converted to human gene names, as

927 above, before running CellphoneDB with statistical analysis on each file.

928

929 Identification of candidate etiologic cell types using CELLEX and CELLECT

930 CELLECT (https://github.com/perslab/CELLECT) and CELLEX

931 (https://github.com/perslab/CELLEX) were used to identify candidate etiological cell types for a 
932 total of 23 traits. The input data for CELLECT is GWAS summary statistics for a given trait and

933 cell type expression specificity (ES) estimates derived from single-cell RNA-seq data. The

934 output is a list of prioritized candidate etiologic cell types for a given trait. ES estimates were

935 calculated using CELLEX (version 1.1), which computes robust estimates of ES by relying on

936 multiple expression specificity measures (for further details see Timshel et. al. ${ }^{74}$ ). CELLEX was

937 run separately on the raw mouse and human (sNuc) gene expression matricies to compute gene

938 expression specificities for each cluster based on the clustering assignment reported above. The

939 resulting cell type specificity matrix was used along with multiple GWAS studies ${ }^{30,75-79}$

940 (Extended Data Table 3) as input for CELLECT $^{74}$ (version 1.1), which was run with default

941 parameters. Significant cell types were identified using a by-trait and by-species Bonferroni $p$ -

942 value threshold of $p<0.05$.

\section{SNP analysis for bulk mRNA-seq cohort}

945 The raw GTC SNP expression data from Infinium OmniExpress-24 Kit was converted to VCF

946 format using Picard version 2.21.6. The pre-processing of the SNP data before phasing and

947 imputation was performed using plink2 (https://www.cog-genomics.org/plink/2.0/). The SNP

948 genotype was then phased and imputed using the Eagle v2.3.5 ${ }^{80}$ and Minimac $3^{81}$ packages,

949 respectively. SNPs were mapped to the NCBI database using the rsnps package

950 (https://CRAN.R-project.org/package=rsnps) and filtered to keep only SNPs that had a minor

951 allele frequency $>0.05$. For plotting gene expression against genotype, bulk RNA sequencing

952 data was TMM normalized using edge ${ }^{82}$. Statistical validation for significance was done using

953 the Wilcoxon rank-sum Test which is a non-parametric test assuming independent samples. 


\section{Statistics}

$956 p$-values for scatterplots were calculated using GraphPad Prism version 8.0 and represent the

957 probability that the slope of the line of best fit is nonzero. All error bars on bar graphs represent

958 standard error. Statistics on proportional composition graphs were calculated using scCODA ${ }^{83}$

959 (version 0.1.2) using the Hamiltonian Monte Carlo sampling method. The model formula used

960 was "Depot + BMI" (human) or "Depot + Diet) (mouse) for all objects in for which both of these

961 covariates were present, or the individual covariate when only a single condition was present.

DATA AVAILABILITY

964 Single cell RNA expression and count data is deposited in the Single Cell Portal (Study

965 \# $\underline{\mathrm{SCP} 1376}$ ) and will be downlodable upon publication. Processed count data for bulk RNA-seq

966 and dge matrices for single cell and single nucleus RNA-seq have been deposited in GEO and

967 will be made public upon publication (Bulk-seq Accession \#GSE174475, sc-RNA-seq/sNuc-sec

968 Acession \#GSE176171), raw sequencing reads for mouse data will additionally be deposited

969 before publication. FASTQ and SNP array files for human samples will be deposited in dbGaP

970 before publication.

972 CODE AVAILABILITY

973 Data analysis pipelines used in this study for processing of raw sequencing data, integration, and

974 clustering can be obtained from https://gitlab.com/rosen-lab/white-adipose-atlas. 


\section{METHODS REFERENCES}

976 52. Katz, A. et al. Quantitative Insulin Sensitivity Check Index: A Simple, Accurate Method

977 for Assessing Insulin Sensitivity In Humans. J. Clin. Endocrinol. Metab. 85, 2402-2410

978 (2000).

979 53. Matthews, D. R. et al. Homeostasis model assessment: insulin resistance and $\beta$-cell

980 function from fasting plasma glucose and insulin concentrations in man. Diabetologia 28,

$981 \quad 412-419(1985)$.

982 54. Macosko, E. Z. et al. Highly Parallel Genome-wide Expression Profiling of Individual 983 Cells Using Nanoliter Droplets. Cell 161, 1202-1214 (2015).

984 55. Drokhlyansky, E. et al. The Human and Mouse Enteric Nervous System at Single-Cell 985 Resolution. Cell 182, 1606-1622.e23 (2020).

986 56. Slyper, M. et al. A single-cell and single-nucleus RNA-Seq toolbox for fresh and frozen 987 human tumors. Nat. Med. 26, 792-802 (2020).

988 57. Delorey, T. M. et al. A single-cell and spatial atlas of autopsy tissues reveals pathology 989 and cellular targets of SARS-CoV-2. bioRxiv (2021) doi:10.1101/2021.02.25.430130.

990 58. Dobin, A. et al. STAR: ultrafast universal RNA-seq aligner. Bioinforma. Oxf. Engl. 29, $991 \quad 15-21(2013)$.

992 59. CellBender remove-background: a deep generative model for unsupervised removal of 993 background noise from scRNA-seq datasets | bioRxiv.

994 https://www.biorxiv.org/content/10.1101/791699v1.

995 60. Lun, A. T. L. et al. EmptyDrops: distinguishing cells from empty droplets in droplet996 based single-cell RNA sequencing data. Genome Biol. 20, 63 (2019). 
997 61. Wolock, S. L., Lopez, R. \& Klein, A. M. Scrublet: Computational Identification of Cell

998 Doublets in Single-Cell Transcriptomic Data. Cell Syst. 8, 281-291.e9 (2019).

999 62. Patro, R., Duggal, G., Love, M. I., Irizarry, R. A. \& Kingsford, C. Salmon provides fast 1000 and bias-aware quantification of transcript expression. Nat. Methods 14, 417-419 (2017).

1001 63. Srivastava, A. et al. Alignment and mapping methodology influence transcript abundance 1002 estimation. Genome Biol. 21, 239 (2020).

1003 64. Soneson, C., Love, M. I. \& Robinson, M. D. Differential analyses for RNA-seq:

1004 transcript-level estimates improve gene-level inferences. F1000Research 4, 1521 (2015).

1005 65. Stuart, T. et al. Comprehensive integration of single-cell data. Cell 177, 1888-1902.e21

1006 (2019).

1007 66. Hafemeister, C. \& Satija, R. Normalization and variance stabilization of single-cell RNA1008 seq data using regularized negative binomial regression. Genome Biol. 20, 296 (2019).

1009 67. Hao, Y. et al. Integrated analysis of multimodal single-cell data. bioRxiv

$1010 \quad 2020.10 .12 .335331(2020)$ doi:10.1101/2020.10.12.335331.

1011 68. Yu, G., Wang, L.-G., Han, Y. \& He, Q.-Y. clusterProfiler: an R Package for Comparing 1012 Biological Themes Among Gene Clusters. OMICS J. Integr. Biol. 16, 284-287 (2012).

1013 69. Littlejohns, T. J. et al. The UK Biobank imaging enhancement of 100,000 participants:

1014 rationale, data collection, management and future directions. Nat. Commun. 11, 2624 (2020).

1015 70. Sudlow, C. et al. UK Biobank: An Open Access Resource for Identifying the Causes of a 1016 Wide Range of Complex Diseases of Middle and Old Age. PLOS Med. 12, e1001779 (2015).

1017 71. Agrawal, S. et al. Association of machine learning-derived measures of body fat 1018 distribution in \&gt;40,000 individuals with cardiometabolic diseases. 2021.05.07.21256854 
1019 https://www.medrxiv.org/content/10.1101/2021.05.07.21256854v1 (2021)

$1020 \quad$ doi:10.1101/2021.05.07.21256854.

1021 72. Kichaev, G. et al. Leveraging Polygenic Functional Enrichment to Improve GWAS

1022 Power. Am. J. Hum. Genet. 104, 65-75 (2019).

1023 73. Pruim, R. J. et al. LocusZoom: regional visualization of genome-wide association scan 1024 results. Bioinforma. Oxf. Engl. 26, 2336-2337 (2010).

1025 74. Timshel, P. N., Thompson, J. J. \& Pers, T. H. Genetic mapping of etiologic brain cell 1026 types for obesity. eLife 9, e55851 (2020).

1027 75. Mahajan, A. et al. Fine-mapping type 2 diabetes loci to single-variant resolution using 1028 high-density imputation and islet-specific epigenome maps. Nat. Genet. 50, 1505-1513 1029 (2018).

1030 76. Loh, P.-R., Kichaev, G., Gazal, S., Schoech, A. P. \& Price, A. L. Mixed-model 1031 association for biobank-scale datasets. Nat. Genet. 50, 906-908 (2018).

1032 77. Finucane, H. K. et al. Partitioning heritability by functional annotation using genome1033 wide association summary statistics. Nat. Genet. 47, 1228-1235 (2015).

1034 78. Teslovich, T. M. et al. Biological, clinical and population relevance of 95 loci for blood 1035 lipids. Nature 466, 707-713 (2010).

1036 79. Bradfield, J. P. et al. A Genome-Wide Meta-Analysis of Six Type 1 Diabetes Cohorts 1037 Identifies Multiple Associated Loci. PLOS Genet. 7, e1002293 (2011).

1038 80. Loh, P.-R. et al. Reference-based phasing using the Haplotype Reference Consortium 1039 panel. Nat. Genet. 48, 1443-1448 (2016).

1040 81. Das, S. et al. Next-generation genotype imputation service and methods. Nat. Genet. 48, $1041 \quad$ 1284-1287 (2016). 
1042 82. Robinson, M. D., McCarthy, D. J. \& Smyth, G. K. edgeR: a Bioconductor package for 1043 differential expression analysis of digital gene expression data. Bioinformatics 26, 139-140 1044 (2010).

1045 83. Büttner, M., Ostner, J., Müller, C., Theis, F. \& Schubert, B. scCODA: A Bayesian model 1046 for compositional single-cell data analysis. bioRxiv 2020.12.14.422688 (2020)

1047 doi:10.1101/2020.12.14.422688.

1048 84. Cawthorn, W. P., Scheller, E. L. \& MacDougald, O. A. Adipose tissue stem cells meet

1049 preadipocyte commitment: going back to the future[S]. J. Lipid Res. 53, 227-246 (2012).

1050 85. Ferrero, R., Rainer, P. \& Deplancke, B. Toward a Consensus View of Mammalian 1051 Adipocyte Stem and Progenitor Cell Heterogeneity. Trends Cell Biol. 30, 937 (2020).

1052

1053

1054 This work was supported by NIH grants RC2 DK116691 to EDR, LTT, AC, OA, and AR, AHA 1055

1056

1057

1058

1059

1060

1061

1062

1063

1064

\section{ACKNOWLEDGEMENTS}

POST14540015 and DoD PRMRP-DAW81XWH to LTT, Broad-BADERC Collaboration Initiative Award (NIH 5P30DK057521) to LTT and EDR, and R01 DK102173 to EDR. MPE is supported by NIH grant F32DK124914. Additional support includes PRIN 2017 (Italian Ministry of University, \#2017L8Z2EM) to AG, THP acknowledges the Novo Nordisk Foundation (unconditional donation to the Novo Nordisk Foundation Center for Basic Metabolic Research; grant number NNF18CC0034900) and the Lundbeck Foundation (Grant number R190-20143904), grants AMP-T2D RFB8b (FNIH) and UM1DK126185 (NIDDK) to MC, Sarnoff Cardiovascular Research Foundation Fellowship to S.A., grants 1K08HG010155 and 1U01HG011719 to A.V.K. from the National Human Genome Research Institute, and a sponsored research agreement from IBM Research to the Broad Institute of MIT and Harvard to 
1065 A.V.K. All single cell library construction and sequencing was performed through the Boston

1066 Nutrition Obesity Research Center Functional Genomics and Bioinformatics Core (NIH

1067 P30DK046200). We thank Christina Usher for artistic support and Miriam Udler for helpful

1068 discussions.

1069

1070 AUTHOR CONTRIBUTIONS

1071 MPE, LTT, and EDR conceived of the project. MPE and EDR wrote the manuscript with

1072 assistance from LTT, CJ, OA, and AR. MPE, ALE, DP, DT, GC, ADV, AS, EM, SS, SL, GPW,

$1073 \mathrm{MLV}$, and AGu performed experiments. GPW, AGu, ZK, JD, CGB, WG, AC, SJL, BTL, DM,

1074 and AT collected samples. MPE, CJ, AMJ, HD, SA, AK, and HS performed computational

1075 analysis. AVK, MC, THP, AGi, OA, and AR provided additional intellectual input.

1076

1077 COMPETING INTEREST DECLARATION

1078 S.A. has served as a scientific consultant to Third Rock Ventures. A.V.K. has served as a

1079 scientific advisor to Sanofi, Amgen, Maze Therapeutics, Navitor Pharmaceuticals, Sarepta

1080 Therapeutics, Novartis, Verve Therapeutics, Silence Therapeutics, Veritas International, Color

1081 Health, Third Rock Ventures, and Columbia University (NIH); received speaking fees from

1082 Illumina, MedGenome, Amgen, and the Novartis Institute for Biomedical Research; and received

1083 a sponsored research agreement from the Novartis Institute for Biomedical Research. M.C. holds

1084 equity in Waypoint Bio and is a member of the Nestle Scientific Advisory Board. A.R. is a co-

1085 founder and equity holder of Celsius Therapeutics, an equity holder in Immunitas Therapeutics

1086 and a scientific advisory board member of Thermo Fisher Scientific, Syros Pharmaceuticals, 
1087 Asimov and Neogene Therapeutics. A.R. is also an employee of Genentech. All other authors

1088 declare no competing interests.

1089

1090 ADDITIONAL INFORMATION

1091 Supplementary information is available for this paper.

1092 Correspondence and requests for materials should be addressed to EDR. 


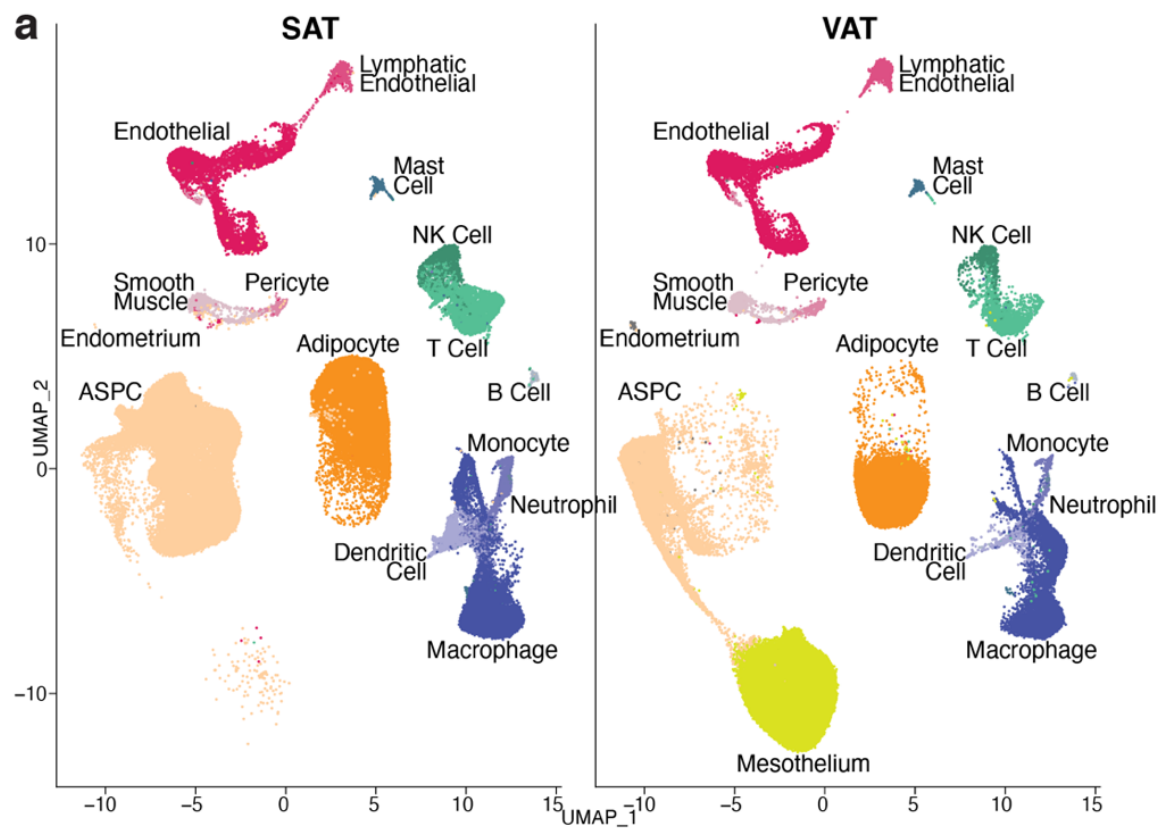

b

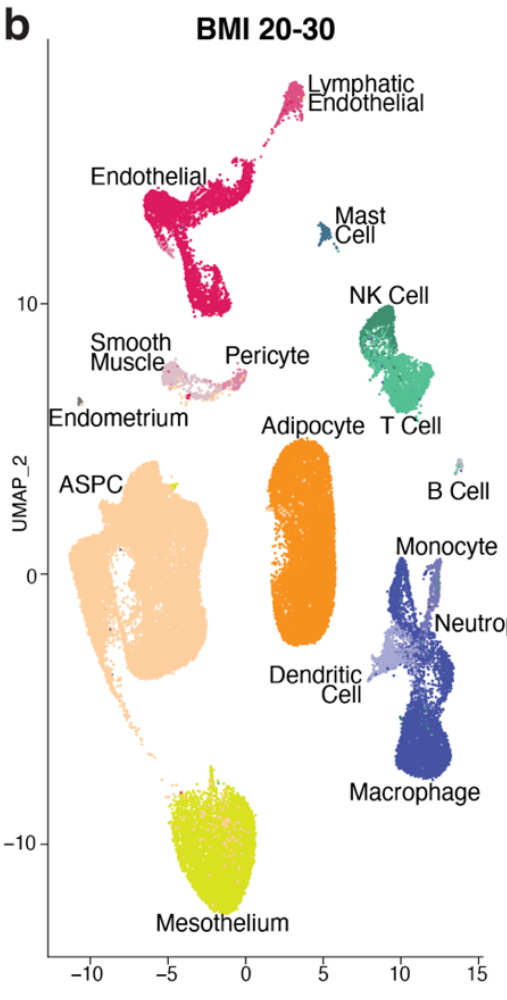

BMI 30-40

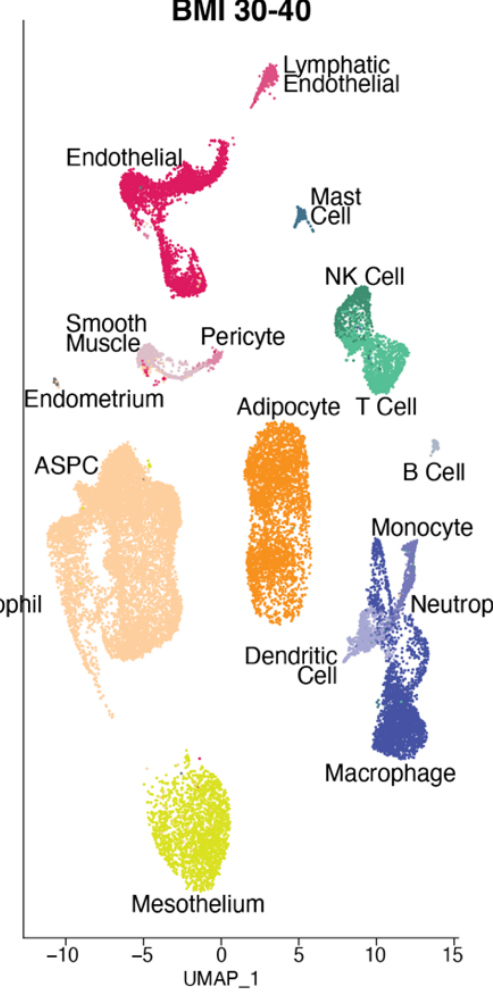

BMI 40-50

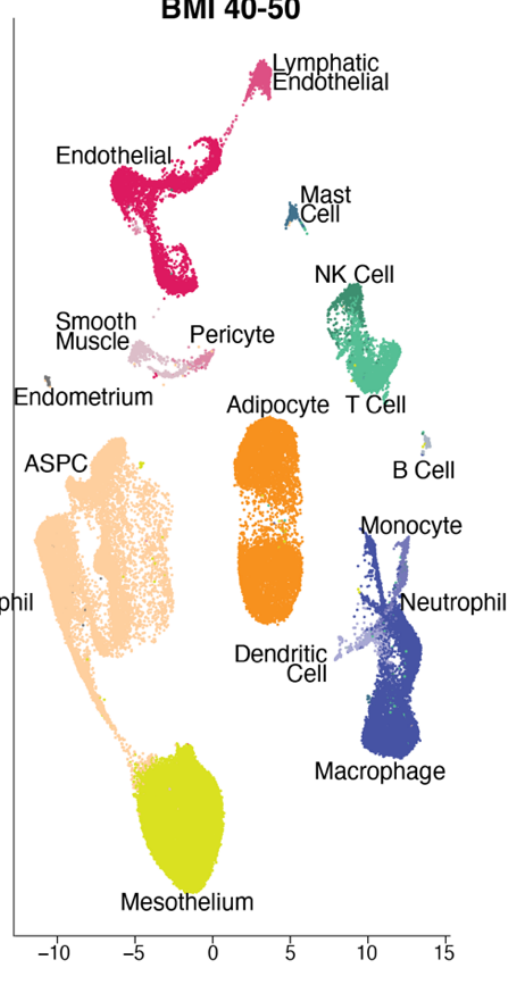

1094 Extended Data Fig. 1. Recovery of human WAT cell types is highly influenced by adipose

1095 depot. a, UMAP projection of all human cells split by depot. b, UMAP projection of all human cells split by BMI range. 

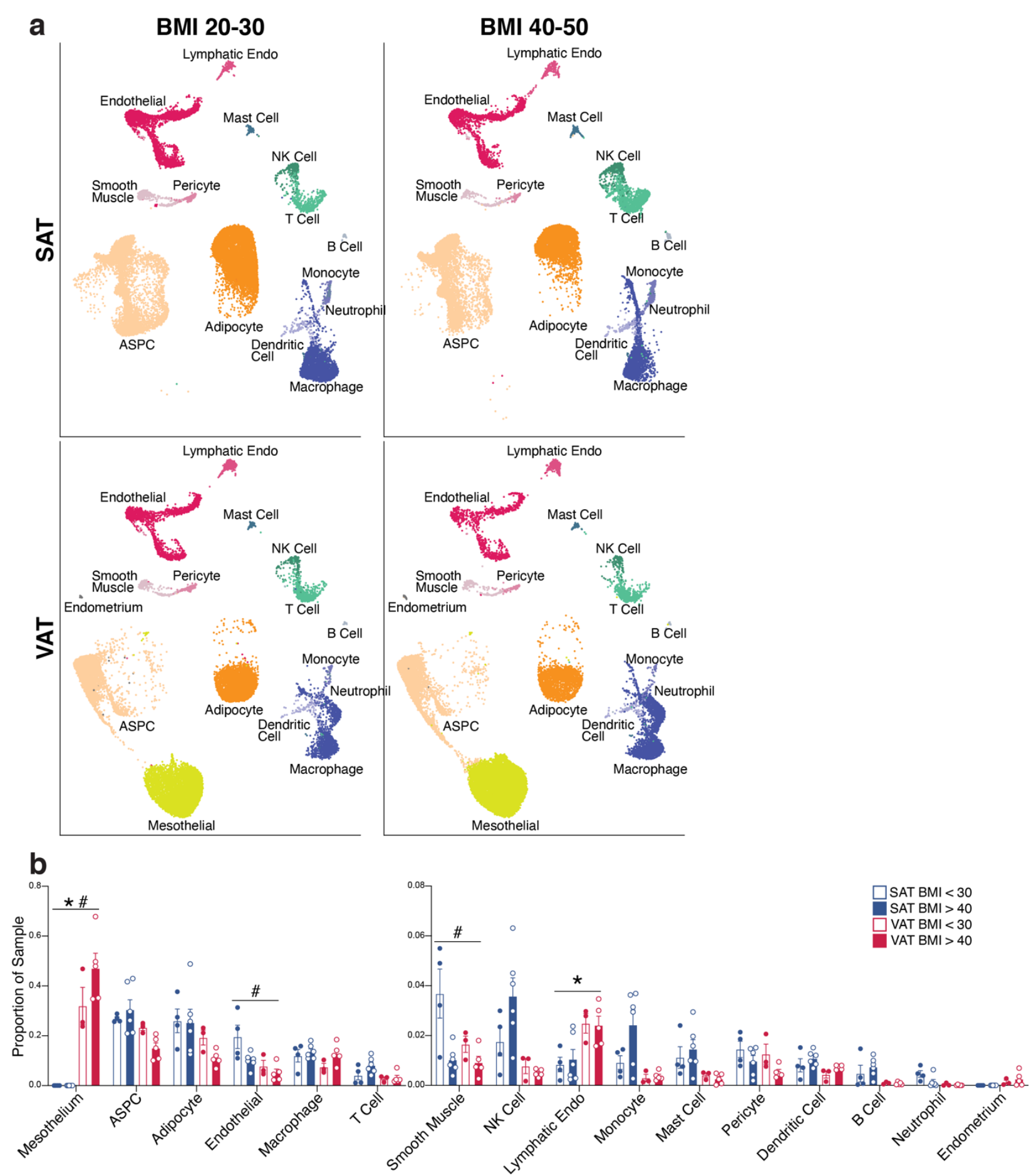

1097 Extended Data Fig. 2. Additional analysis of the effects of depot and BMI on human WAT

1098 populations. a, UMAP projections of cells from the lowest and highest BMI ranges in the 
1099 dataset, split by depot. To facilitate comparison, samples were randomly subset to contain the

1100 same number of cells in each plot $(\mathrm{n}=20,339)$. $\mathbf{b}$, Graph showing the proportion of sNuc-seq

1101 cells in each cluster per sample, split by depot and BMI. For bar graphs, * indicates credible

1102 depot effect and \# indicates credible BMI effect, calculated using dendritic cells as reference. 
bioRxiv preprint doi: https://doi.org/10.1101/2021.11.09.466968; this version posted November 11,2021 . The copyright holder for this preprint (which was not certified by peer review) is the author/funder, who has granted bioRxiv a license to display the preprint in perpetuity. It is made available under aCC-BY-NC-ND 4.0 International license.
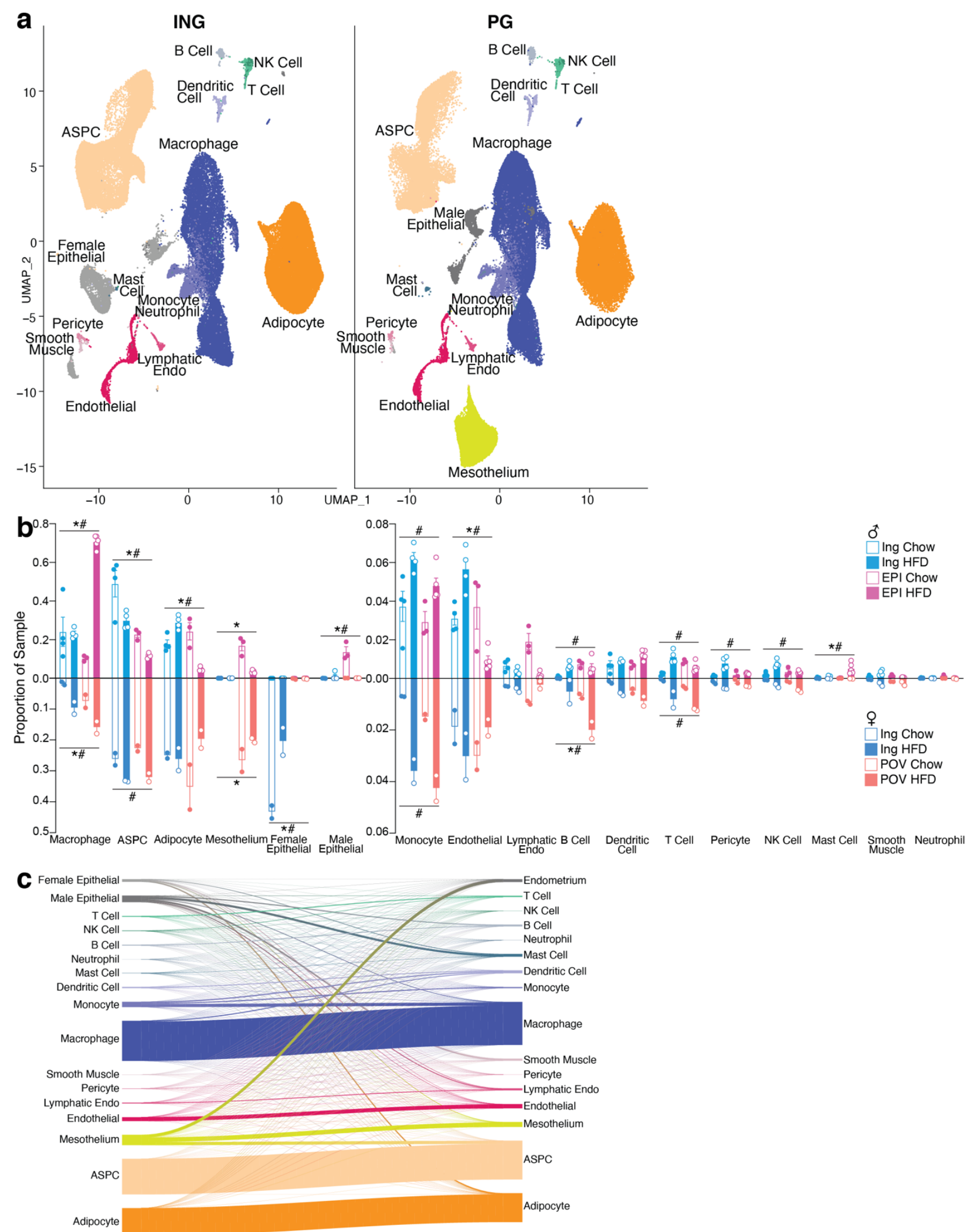


\section{Extended Data Fig. 3. Additional analysis of the effects of depot and diet on mouse WAT}

1104 populations and association with human WAT populations. a, UMAP projection of all mouse

1105 WAT cells split by depot. b, Proportion of cells in each cluster per sample, split by sex as well as

1106 by depot and diet. c, Riverplot showing the relationship between mouse and human clusters.

1107 Mouse cells were mapped onto human sNuc-seq cells using multimodal reference mapping. The

1108 riverplot represents the relationship between manually assigned mouse cluster and mapped

1109 human cluster for every mouse cell. For bar graph, error bars represent SEM, * indicates credible

1110 depot effect and \# indicates credible diet effect, calculated using dendritic cells as reference. 


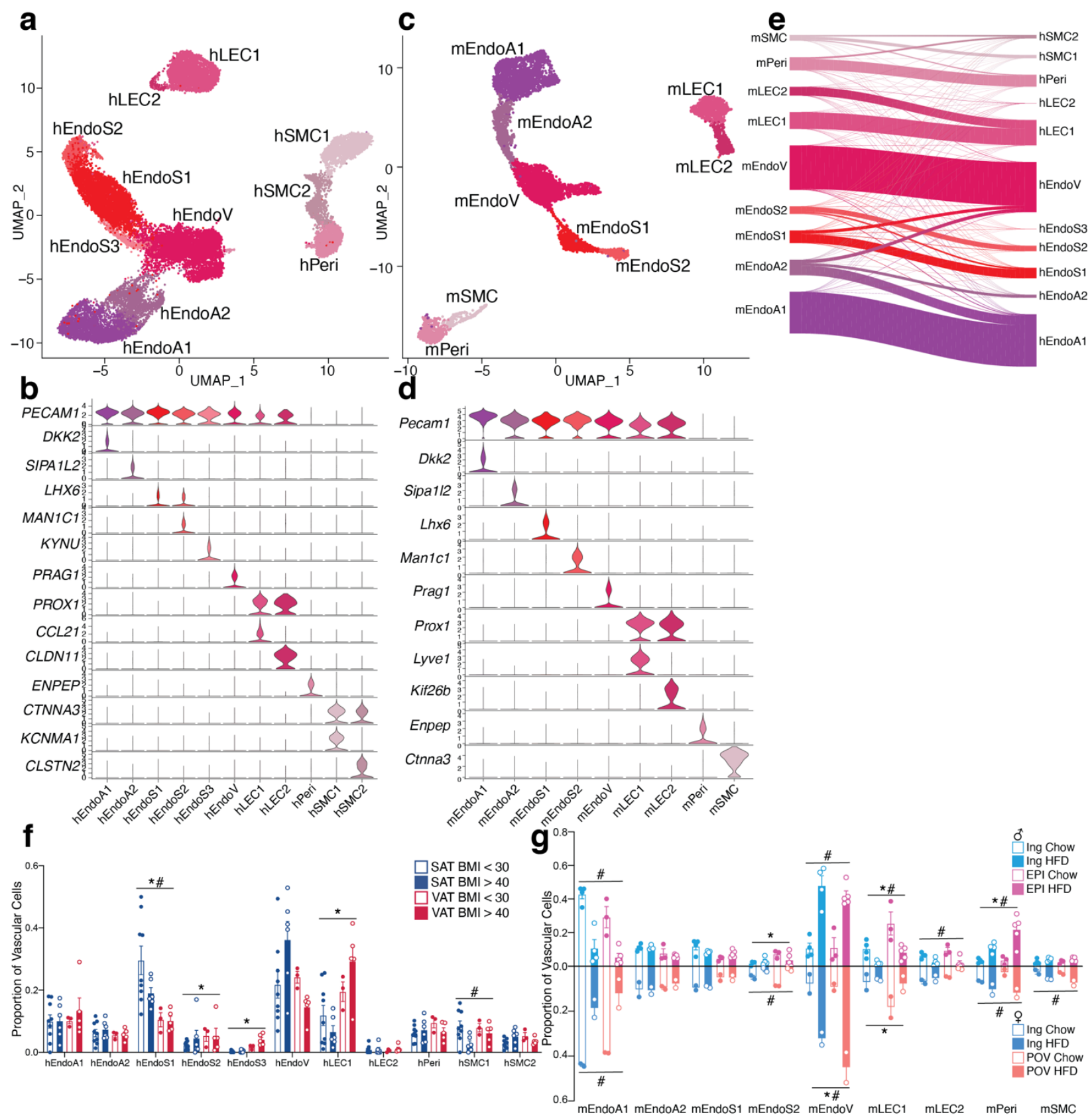

1111 Extended Data Fig. 4. Highly similar vascular cells in human and mouse WAT. a, UMAP

1112 projection of 22,734 human vascular cells. b, Marker genes for 11 distinct clusters of human

1113 WAT vascular cells. c, UMAP projection of 7,632 mouse vascular cells. d, Marker genes for 9

1114 distinct clusters of mouse WAT vascular cells. e, Riverplot showing the correlation between

1115 annotated mouse and human vascular clusters based on multimodal reference mapping for each 
1116 mouse cell. f-g, Bar graphs showing the proportion of cells in each cluster per sample split by

1117 depot and BMI for human (f) and depot, diet, and sex for mouse (g). For bar graphs, error bars

1118 represent SEM, * indicates credible depot effect and \# indicates credible BMI/diet effect,

1119 calculated using hEndoA2 (human) and mEndoA2 (mouse) as reference. 
bioRxiv preprint doi: https://doi.org/10.1101/2021.11.09.466968; this version posted November 11,2021 . The copyright holder for this preprint (which was not certified by peer review) is the author/funder, who has granted bioRxiv a license to display the preprint in perpetuity. It is made available under aCC-BY-NC-ND 4.0 International license.
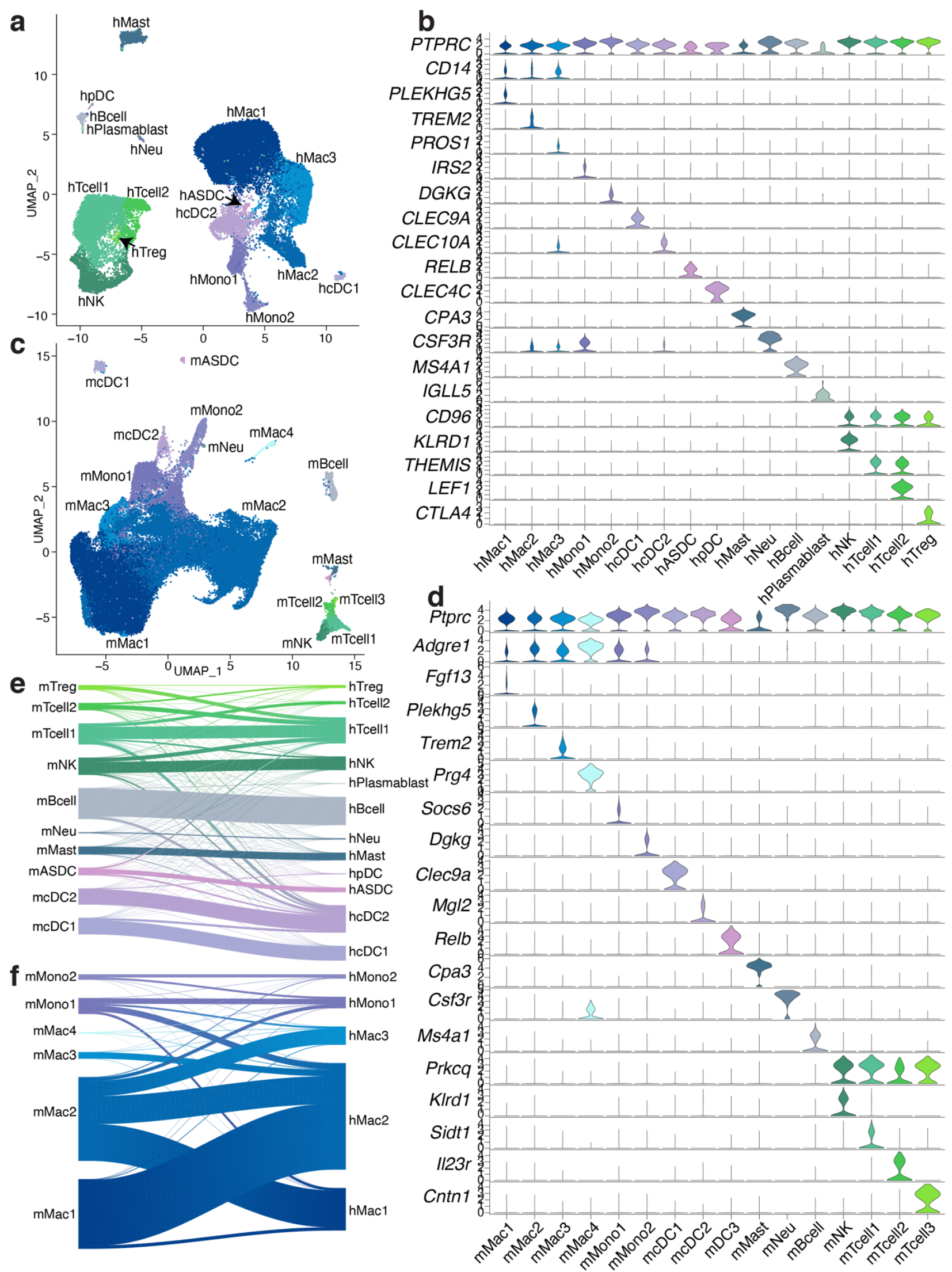
1120 Extended Data Fig. 5. Comparison of immune cells in human and mouse WAT. a, UMAP

1121 projection of 34,268 immune cells from human WAT. b, Marker genes for human immune cell

1122 clusters. c, UMAP projection of 70,547 immune cells from mouse WAT. d, Marker genes for

1123 mouse immune cell clusters. e-f, Riverplots showing the correlation between annotated mouse

1124 cluster and mapped human cluster for mouse (e) dendritic cells, mast cells, neutrophils, B cells,

1125 NK cells, and T cells and (f) monocytes and macrophages. 

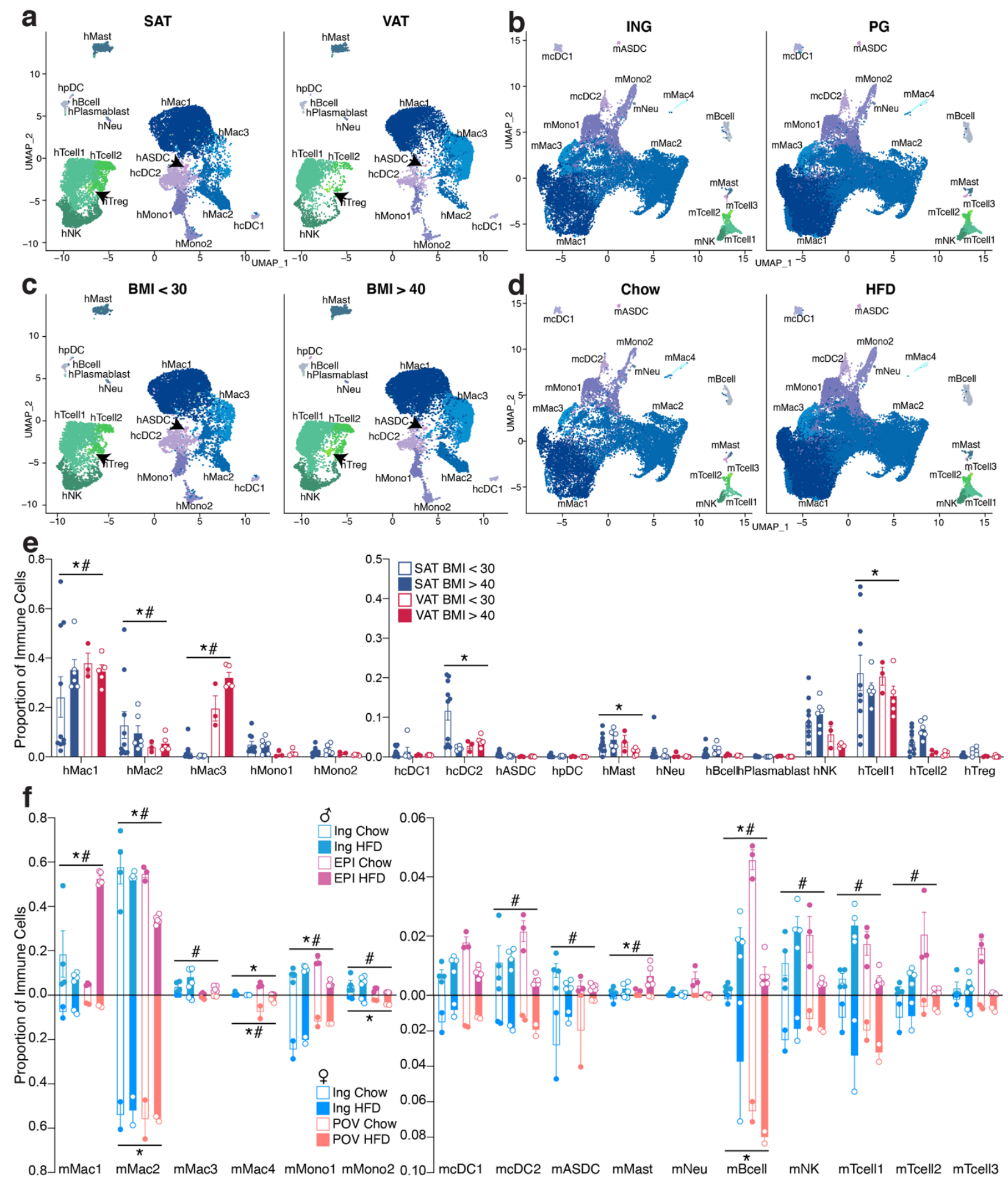

Extended Data Fig. 6. Human and mouse immune cells are differentially regulated by

1127 depot and BMI/diet. a-b, UMAP projections of human (a) and mouse (b) WAT immune cells 1128 split by depot. c-d, UMAP projections of human (c) and mouse (d) WAT immune cells split by 
1129 BMI (c) and diet (d). e-f, Bar graphs showing the proportion of cells in each cluster per sample

1130 split by depot and BMI for human (e) and depot, diet, and sex for mouse (f). For bar graphs,

1131 error bars represent SEM, * indicates credible depot effect and \# indicates credible BMI/diet

1132 effect, calculated using hMono2 (human) and $\mathrm{mcDC} 1$ (mouse) as reference. 

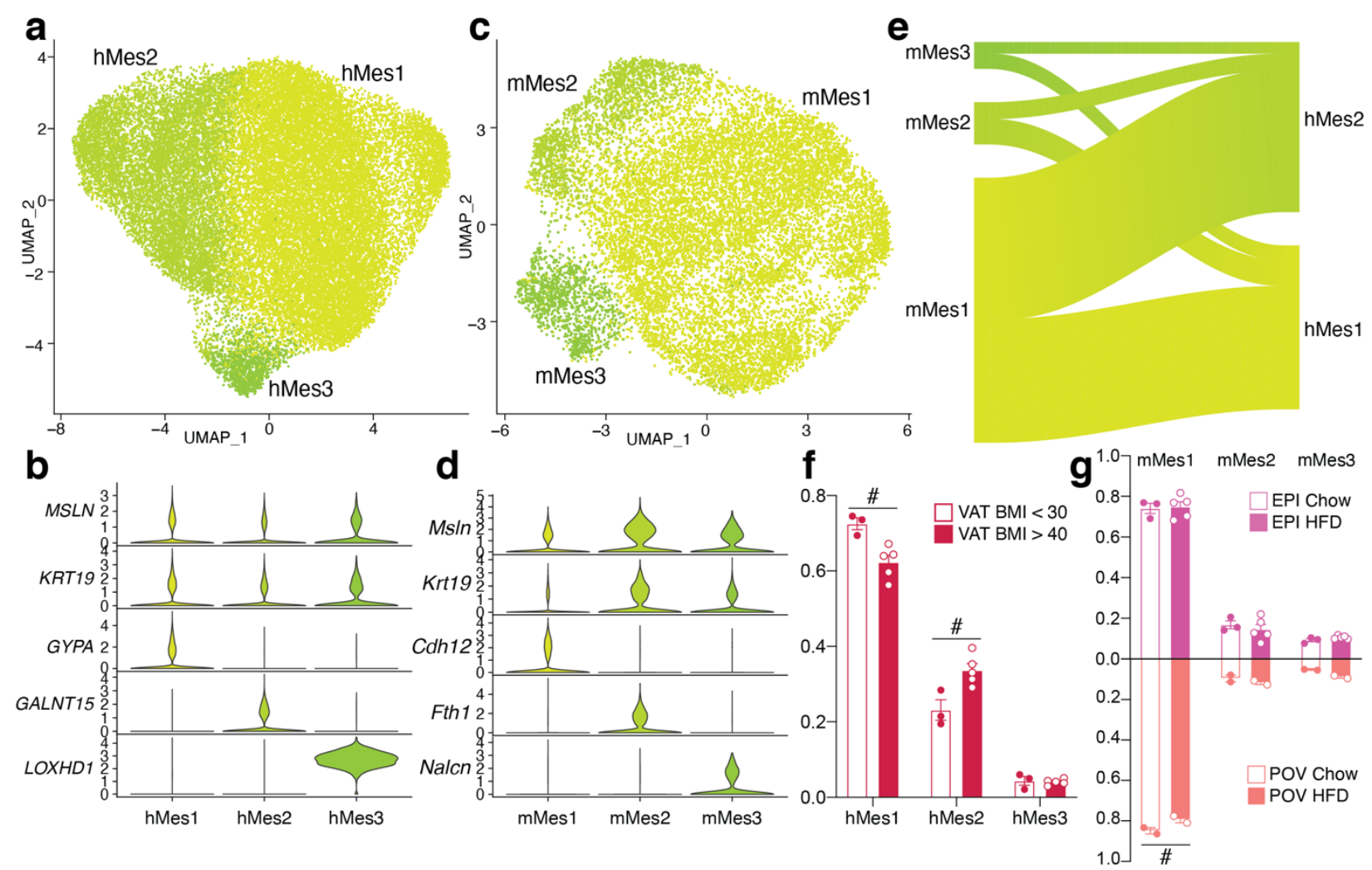

1133 Extended Data Fig. 7. Subpopulations of human and mouse mesothelial cells. a, UMAP

1134 projection of 30,482 human mesothelial cells. b, Marker genes for distinct human mesothelial

1135 populations. c, UMAP projection of 14,947 mouse mesothelial cells. d Marker genes for distinct

1136 mouse mesothelial populations. e, Riverplots showing relationship of mouse and human

1137 mesothelial clusters. f-g, Proportion of cells in each cluster per sample, split by BMI for human

1138 (f) and diet and sex for mouse (g). Error bars represent SEM, \# indicates credible BMI/diet

1139 effect, calculated using hMes3 (human) and mMes1 (mouse) as reference. 


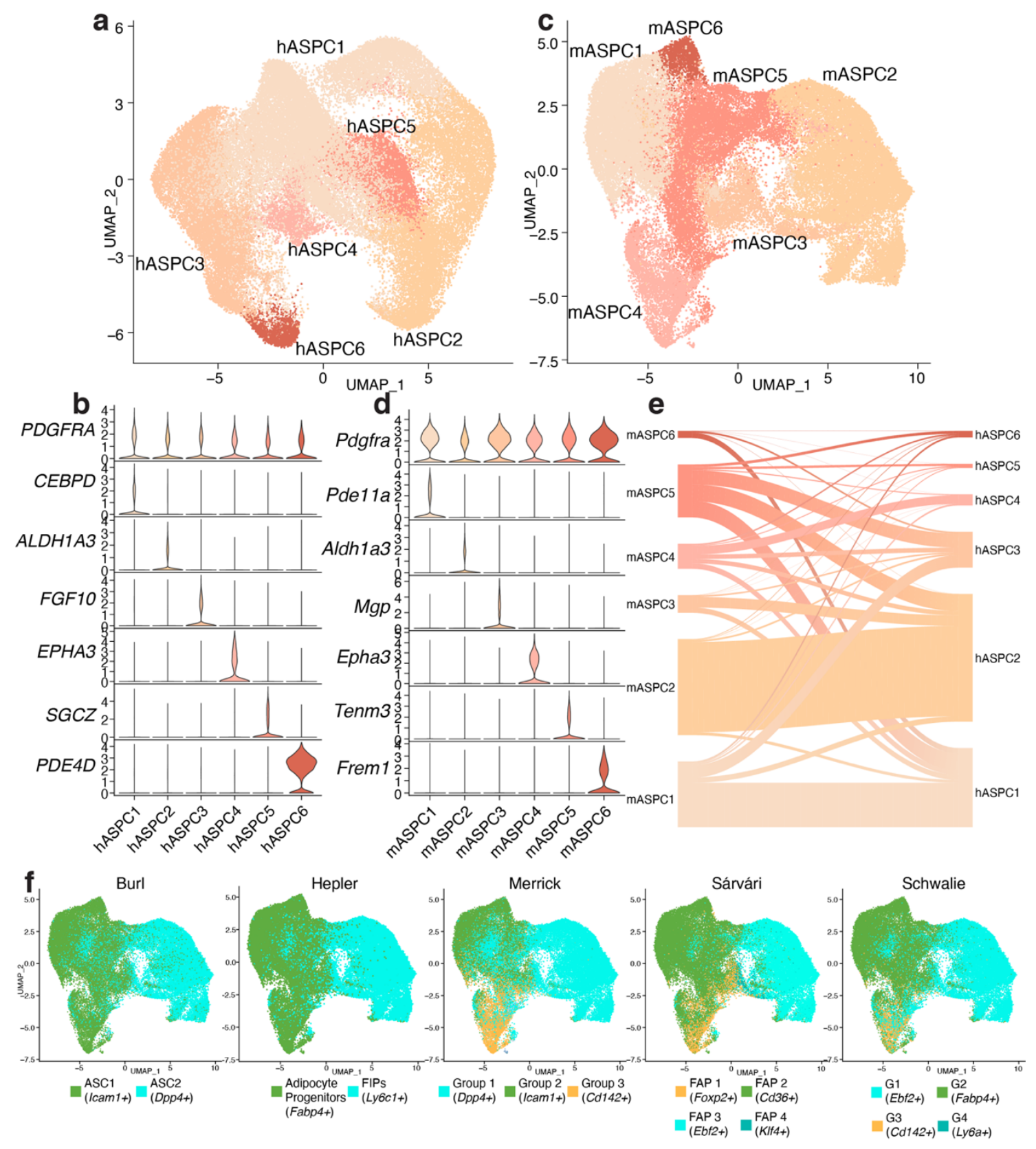

1140 Extended Data Fig. 8. Human and mouse ASPCs share commonalities with previously

1141 reported subtypes. a, UMAP projection of 52,482 human ASPCs. b, Marker genes for distinct

1142 ASPC subpopulations. c, UMAP projection of 51,227 mouse ASPCs. d, Marker genes for

1143 distinct ASPC subpopulations. e, Riverplot depicting the relationship between mouse and human

1144 ASPC clusters. f, Reference mapping of ASPC cell types reported by other groups onto the

1145 mouse ASPCs from this paper. 


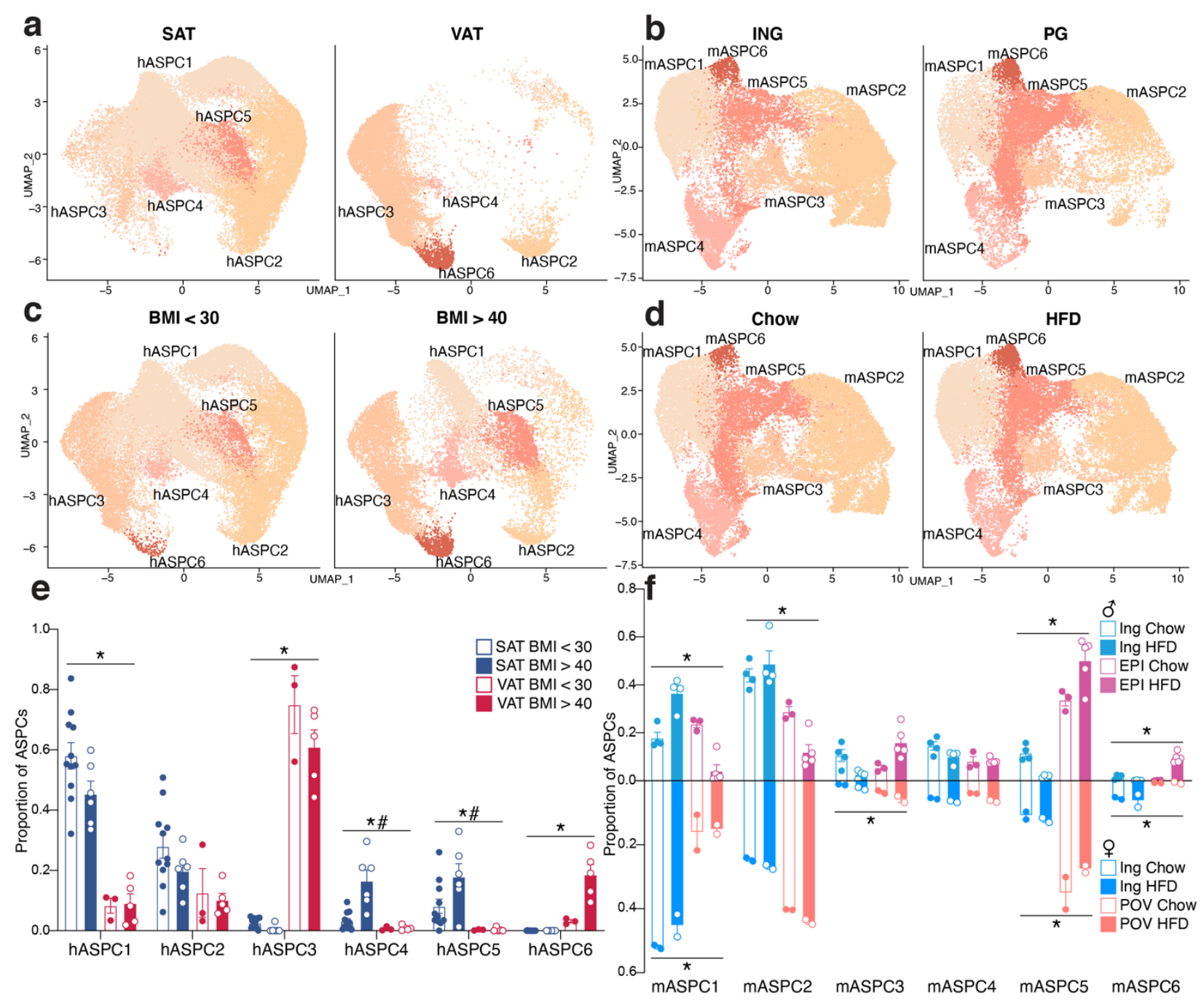

1146 Extended Data Fig. 9. Human ASPCs exhibit strong depot dependency while mouse ASPCs

1147 are dependent on both depot and diet. a-b, UMAP projections of human (a) and mouse (b)

1148 ASPCs split by depot. c-d, UMAP projections of human (c) and mouse (d) ASPCs split by

1149 BMI/diet. e-f, Proportion of ASPC cells in each cluster per sample split by depot and BMI for

1150 human (e) and depot, diet, and sex for mouse (f). For bar graphs, error bars represent SEM, *

1151 indicates credible depot effect and \# indicates credible BMI/diet effect, calculated using hASPC2

1152 (human) and mASPC4 (mouse) as reference. 

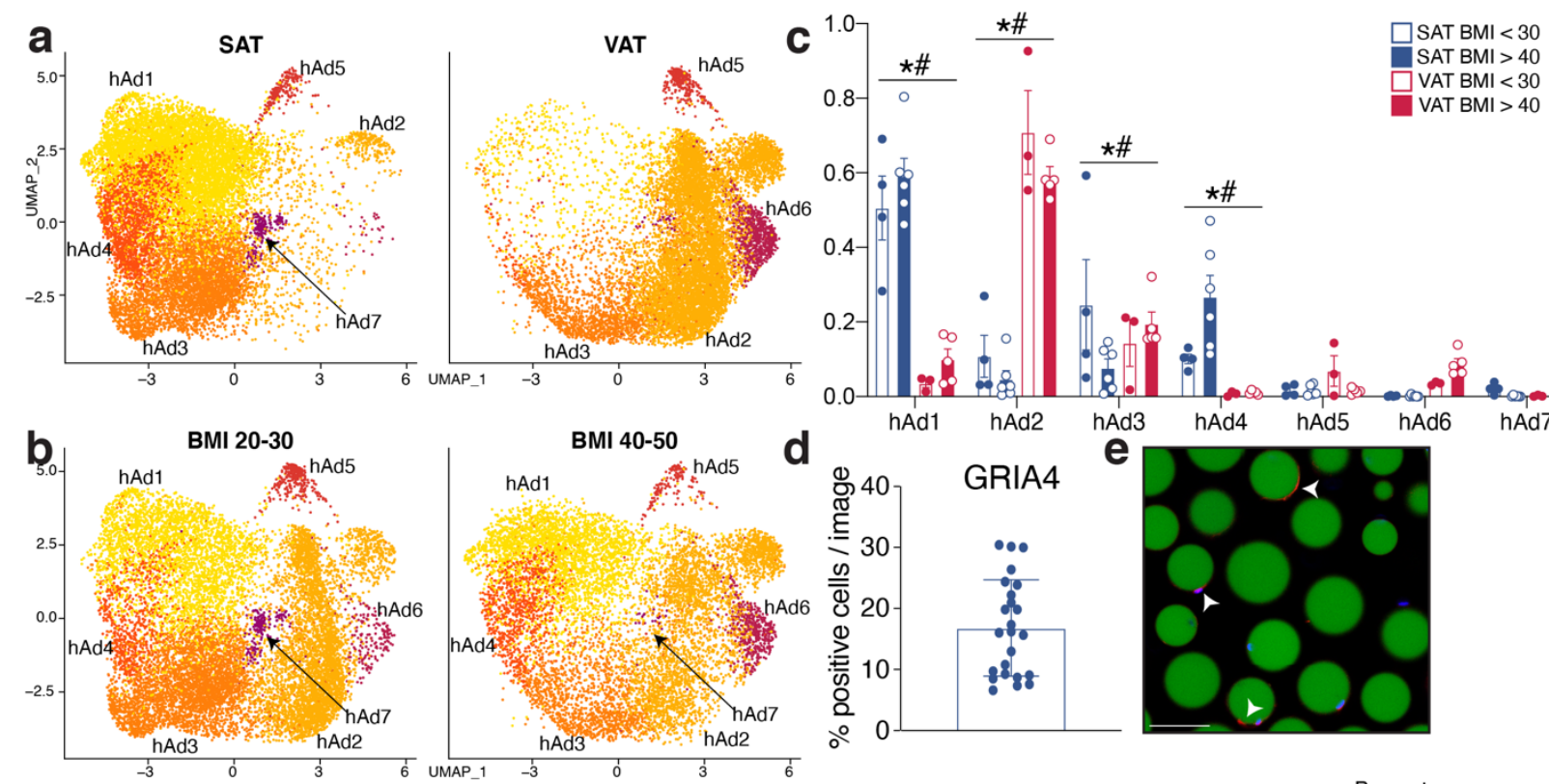

d
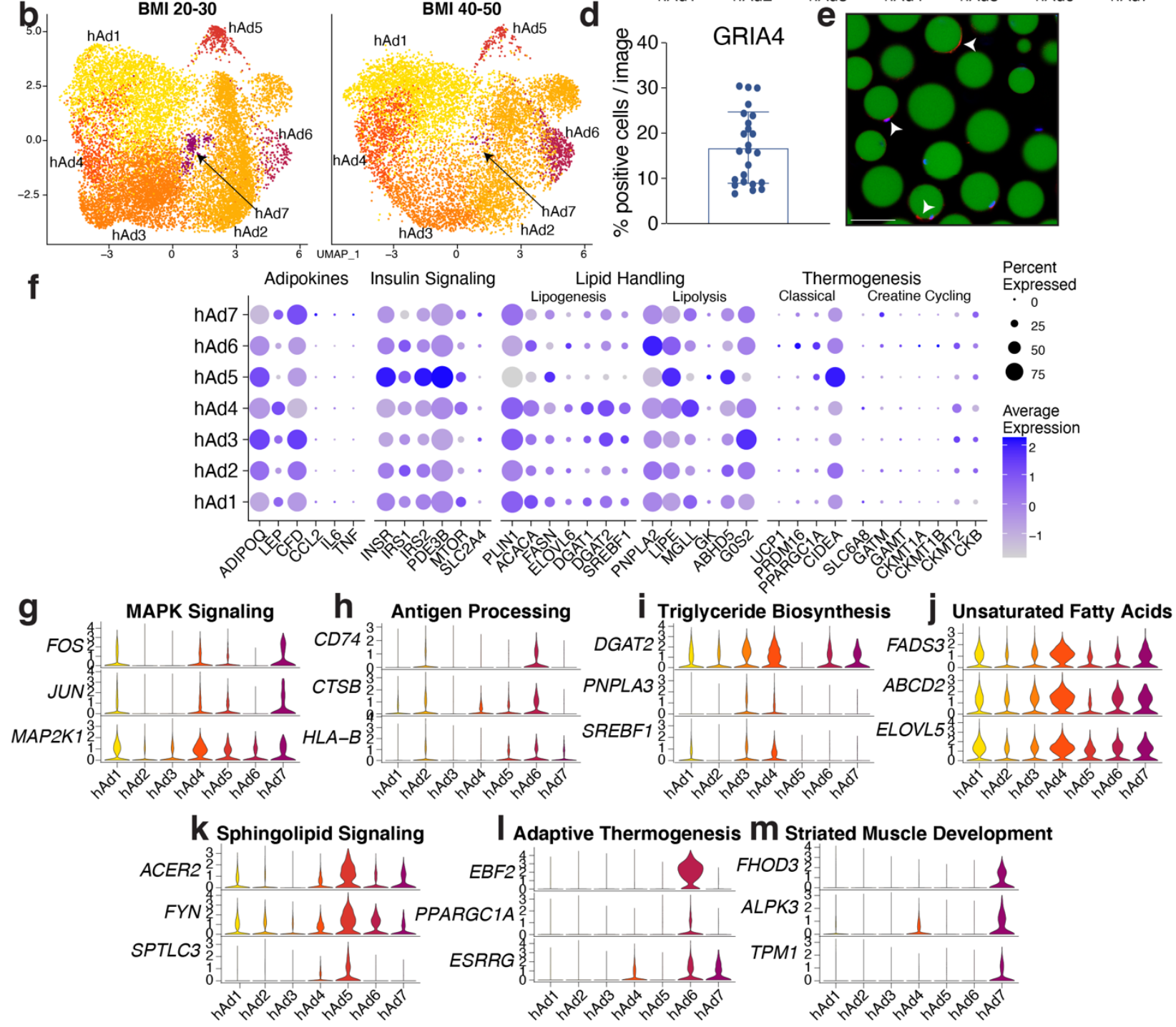

1153 Extended Data Fig. 10. Human adipocyte subtypes are highly dependent on depot and may

1154 be responsible for distinct functions. a-b, UMAP projections of human white adipocytes split

1155 by depot (a) and BMI (b). c, Proportion of cells in each human cluster by sample split by depot 
1156 and BMI. d, Quantification of immunofluorescence analysis of GRIA4+ cells in mature human

1157 adipocytes from two individuals. Each dot represents an image. e, Representative images of

1158 GRIA4+ cells. f, Expression of genes associated with adipokine secretion, insulin signaling, lipid

1159 handling, and thermogenesis across human adipocyte subclusters. g-m, Expression of genes

1160 associated with GO or KEGG pathways indicative of individual human adipocyte subclusters.

1161 For bar graph, error bars represent SEM, * indicates credible depot effect and \# indicates

1162 credible BMI effect, calculated using hAd5 as reference. 


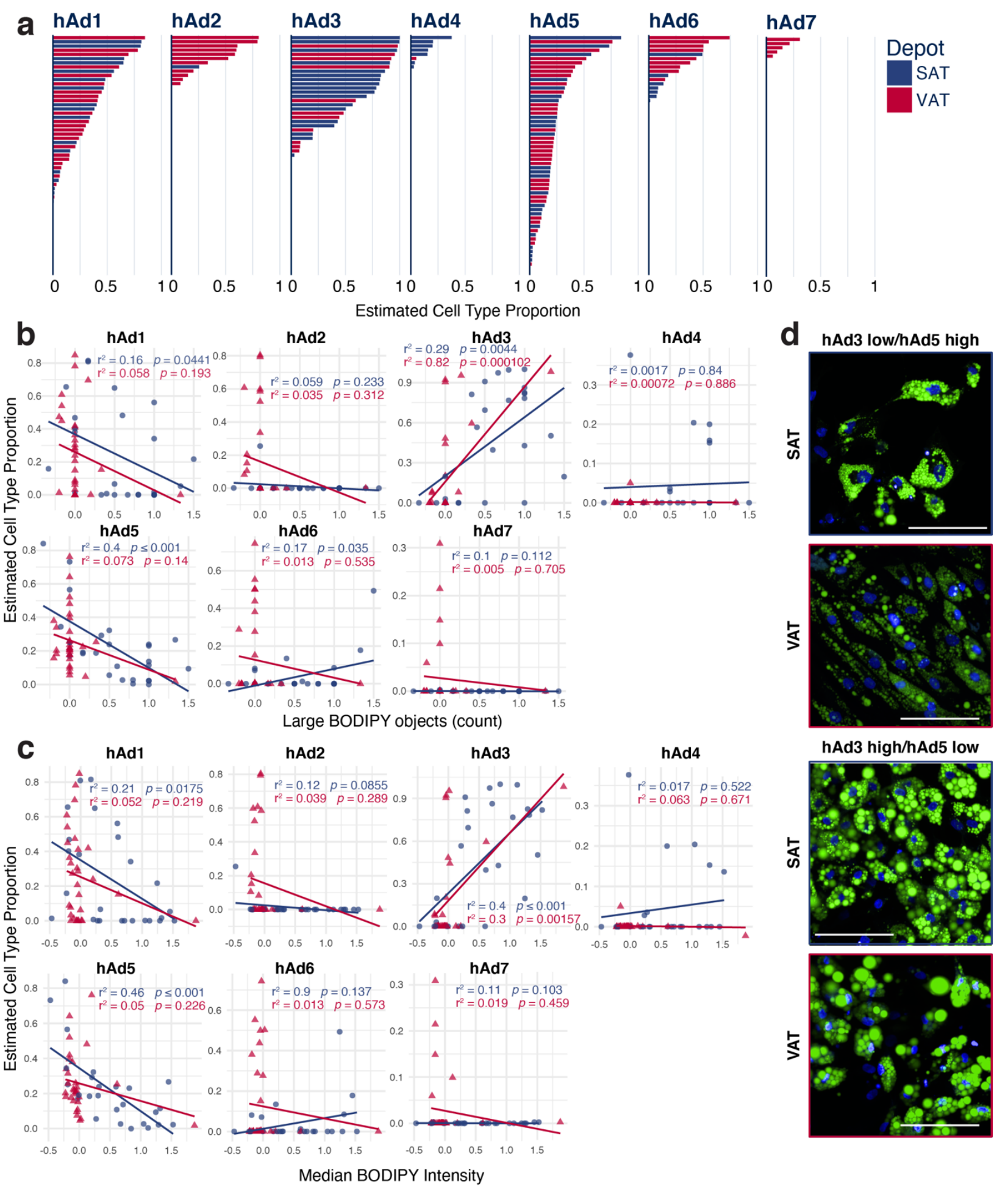

1163 Extended Data Fig. 11. Human adipocytes differentiated ex vivo recapitulate many of the

1164 adipocyte subclusters found in vivo. a, Plot of estimated cell type proportion in ex vivo

1165 adipocyte cultures differentiated from subcutaneous or visceral preadipocytes for 14 days, 
1166 ordered by estimated proportion. b-c, Scatterplots showing the relationship between estimated

1167 cell type proportion and the LipocyteProfiler-calculated features Large BODIPY objects (b) and

1168 Median BODIPY Intensity (c). d, Representative images of hAd3 low/hAd5 or hAd3 high hAd5

1169 low in vitro differentiated cultures. Green represents BODIPY staining, blue represents Hoechst

1170 staining. 

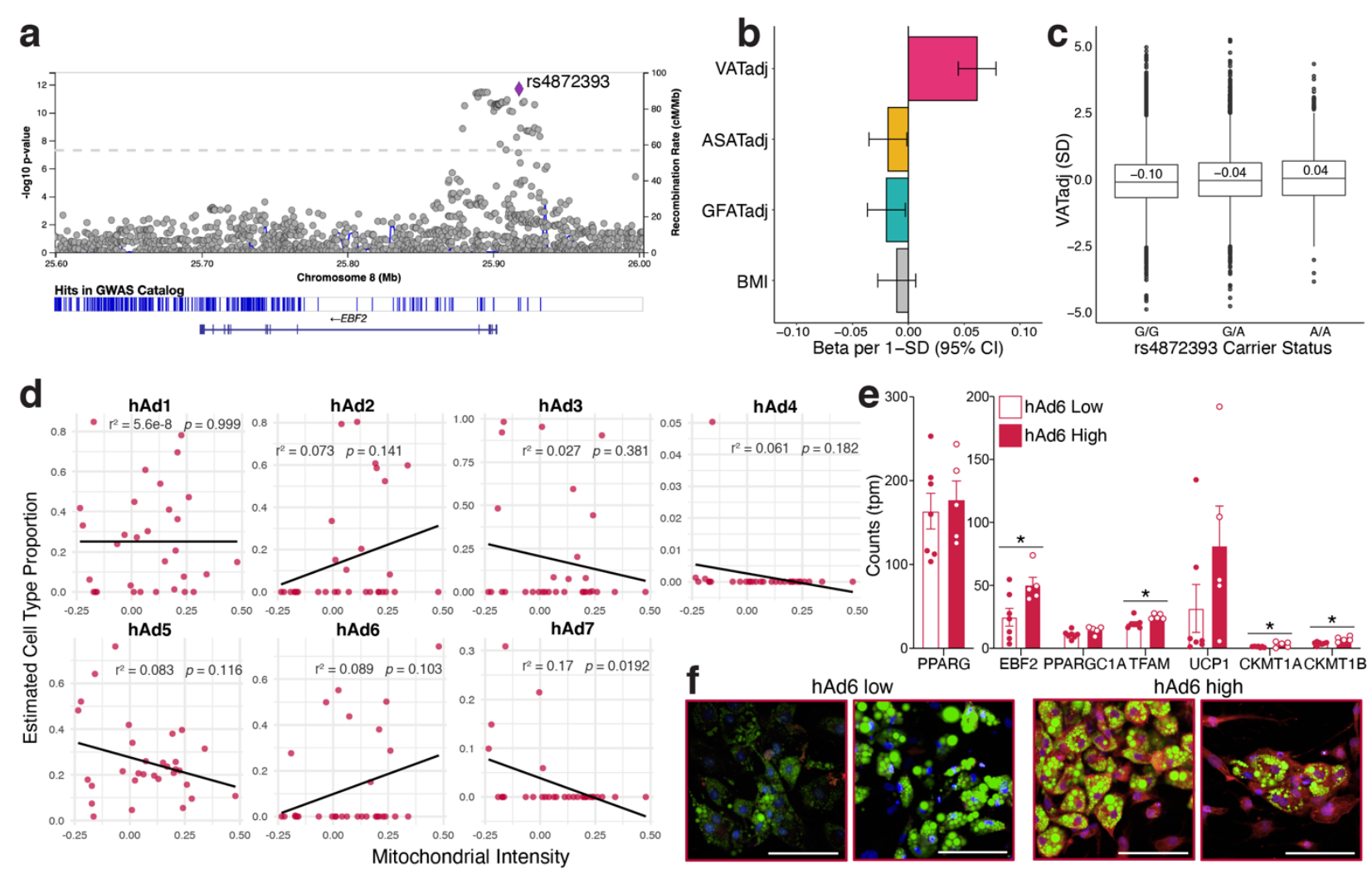

g
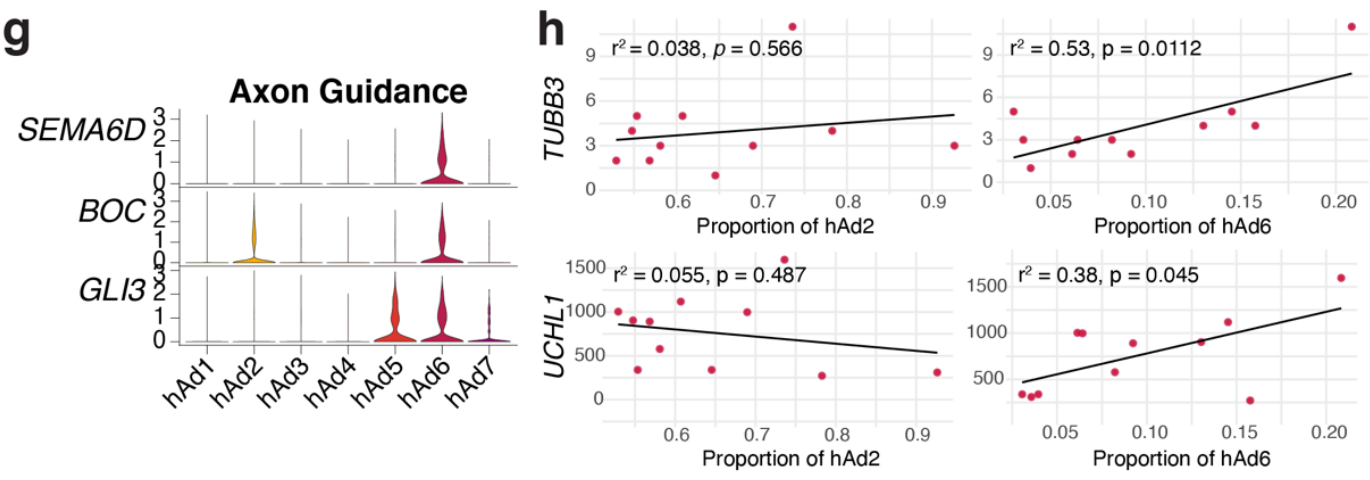

Extended Data Fig. 12. Visceral-specific adipocyte subpopulation hAd6 is associated with

1172 thermogenic traits. a, Regional visualization of associations of common genetic variants near

1173 EBF2 with VATadj. b, Association of rs4872393 with VATadj, ASATadj, GFATadj, and BMI

1174 per minor allele A; $\mathrm{n}=37,641$. $\mathbf{c}$, VATadj raw data plotted according to rs4872393 carrier status;

$1175 \mathrm{n}=36,185$. d, Scatterplot showing the relationship between estimated cell type proportion and

1176 the LipocyteProfiler calculated feature Mitochondrial Intensity in visceral samples. e, Expression

1177 of mitochondrial and thermogenic genes in visceral in vitro differentiated adipocytes stratified by 
1178 estimated hAd6 proportion and matched for amount of differentiation using PPARG levels. f,

1179 Representative images of hAd6 low and high visceral in vitro differentiated cultures. Green

1180 represents BODIPY staining, red represents MitoTracker staining, and blue represents Hoechst

1181 staining. g, Violin plot of sNuc-seq data showing axon guidance genes in adipocyte subclusters.

$1182 \mathbf{h}$, Scatterplots showing the relationship between calculated proportion of visceral subpopulations

1183 hAd2 and hAd6 and expression of pan-neuronal markers on the ambient RNA of individual

1184 visceral sNuc-seq samples. For bar graph, error bars represent SEM, ${ }^{*}, p<.05, * *, p<.01$. 
bioRxiv preprint doi: https://doi.org/10.1101/2021.11.09.466968; this version posted November 11,2021 . The copyright holder for this preprint (which was not certified by peer review) is the author/funder, who has granted bioRxiv a license to display the preprint in perpetuity. It is made available under aCC-BY-NC-ND 4.0 International license.
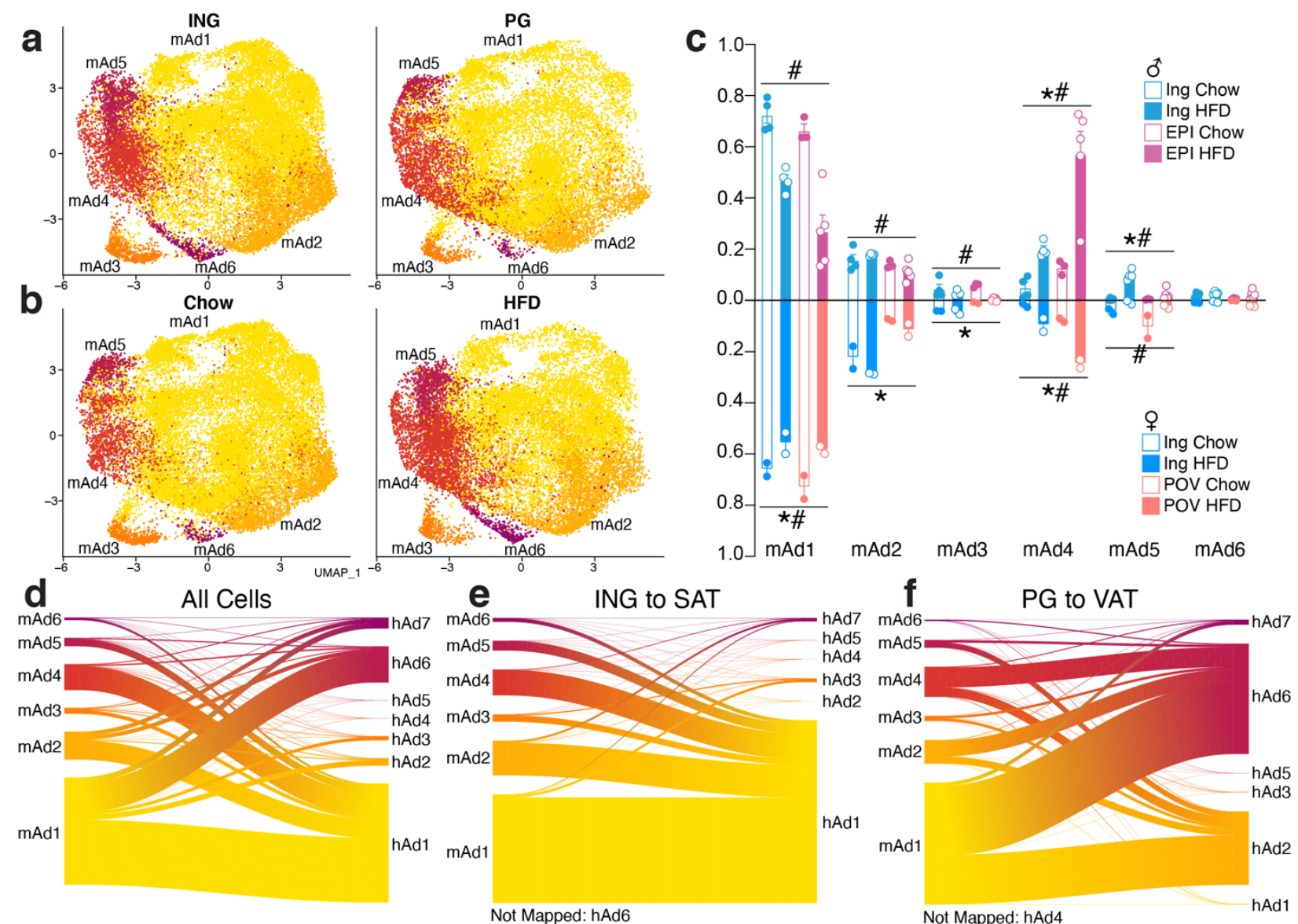

g
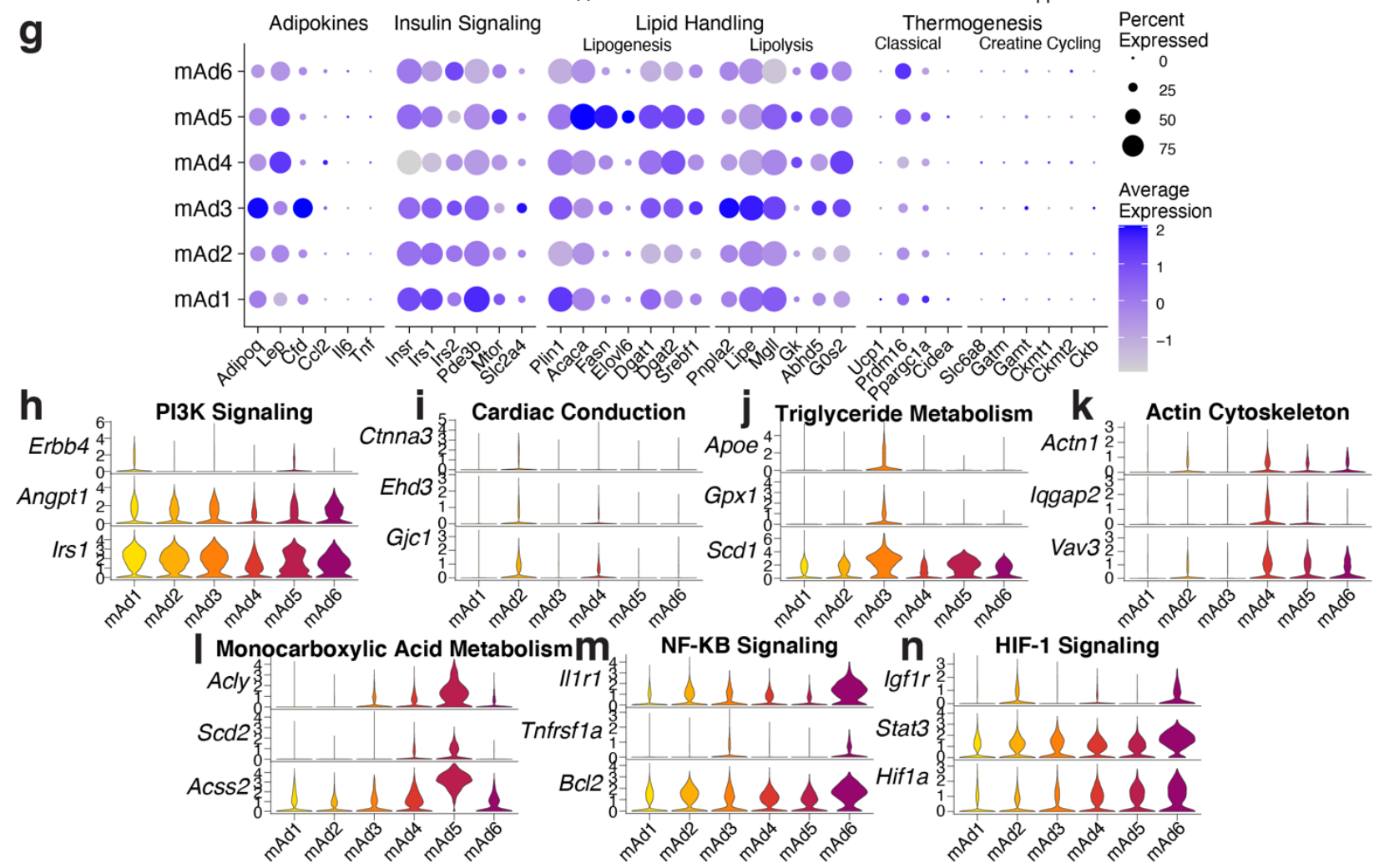


\section{Extended Data Fig. 13. Mouse adipocytes appear to have distinct functionality but are not}

1186 analogous to human adipocyte subpopulations. a-b, UMAP projections of mouse adipocytes

1187 split by depot (a) and diet (b). c, Proportion of cells in each mouse cluster per sample split by

1188 depot, diet, and sex. d, Expression of genes associated with known adipocyte functions in mouse

1189 adipocyte subclusters. e-k, Expression of genes associated with GO or KEGG pathways

1190 indicative of individual mouse adipocyte subclusters. I-n, Riverplots of mouse cells showing the

1191 association between mouse and human adipocyte clusters from both subcutaneous and visceral

1192 depots (I), subcutaneous (ING and SAT) adipocytes only (m) or visceral (PG and VAT)

1193 adipocytes only (n). For depot comparisons, both mouse query objects and human reference

1194 objects were subset to the respective depot before mapping. For bar graph, error bars represent

1195 SEM, * indicates credible depot effect and \# indicates credible diet effect, calculated using

1196 mAd6 as reference. 

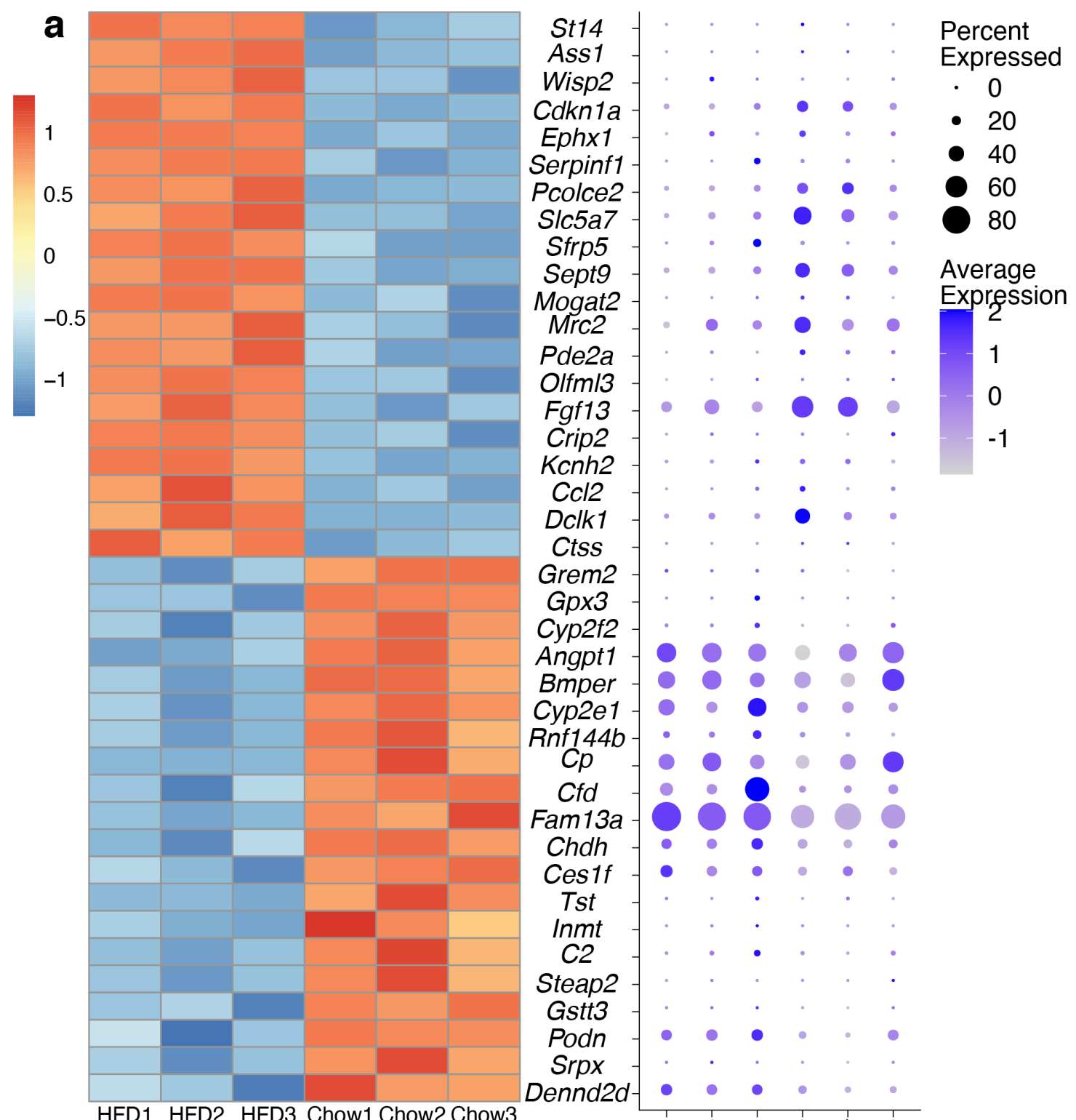

b
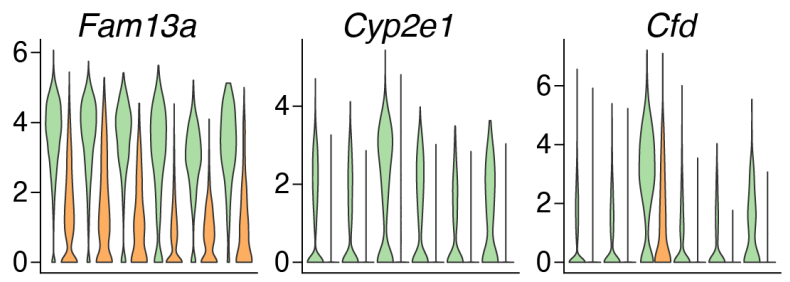

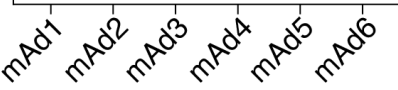

$\mathrm{Ccl} 2$

Dclk1

Ctss

Grem2

Gpx3

Cyp2f2

Angpt1

Bmper

Cyp2e1

$\operatorname{Rnf} 144 b$

Cfd

Fam13a

Chdh

Ces1f

Tst

Inmt

Steap2

Gstt3

Podn

Srpx

Dennd2d

HFD1 HFD2 HFD3 Chow1 Chow2 Chow3

Rnf144b
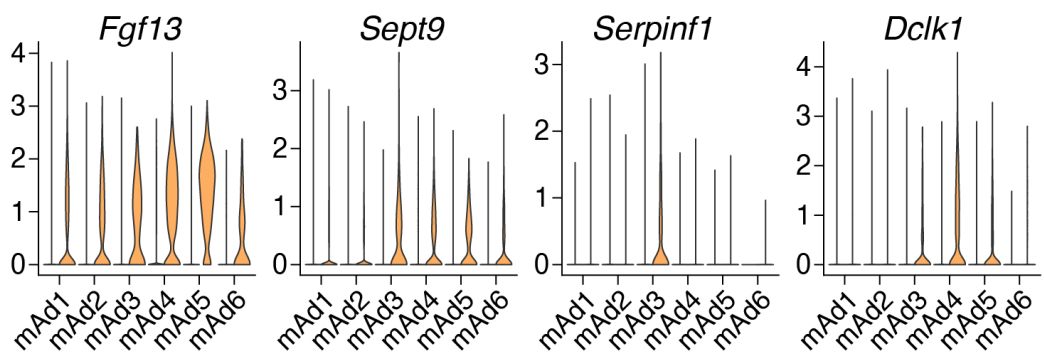


\section{Extended Data Fig. 14. Adipocyte gene expression changes during high fat diet result from}

\section{8 both changes in abundance of adipocyte subtypes and from expression changes within}

1199 subclusters. a, (left) Heatmap depicting expression of the top 20 most up- and down-regulated

1200 genes in adipocytes after HFD feeding, as determined by bulk sequencing of TRAP-isolated

1201 adipocyte RNA. On the right, the same genes are plotted onto the mouse adipocyte subclusters to

1202 determine cluster specificity. b, Selected genes from a are plotted onto mouse adipocyte

1203 subclusters and split by diet. 


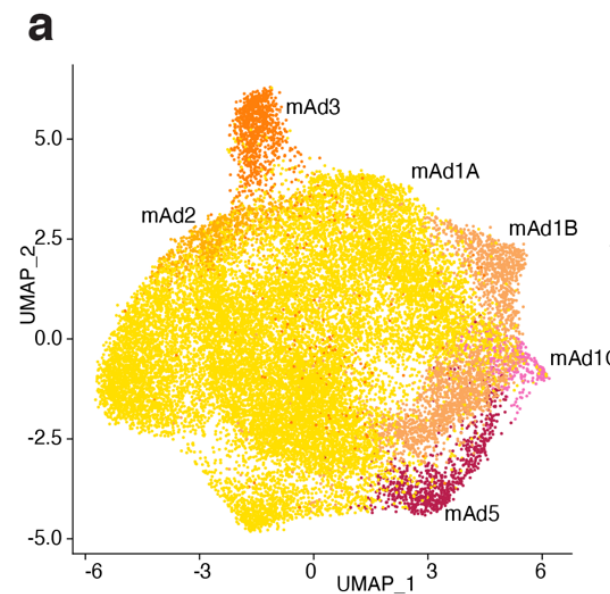

d

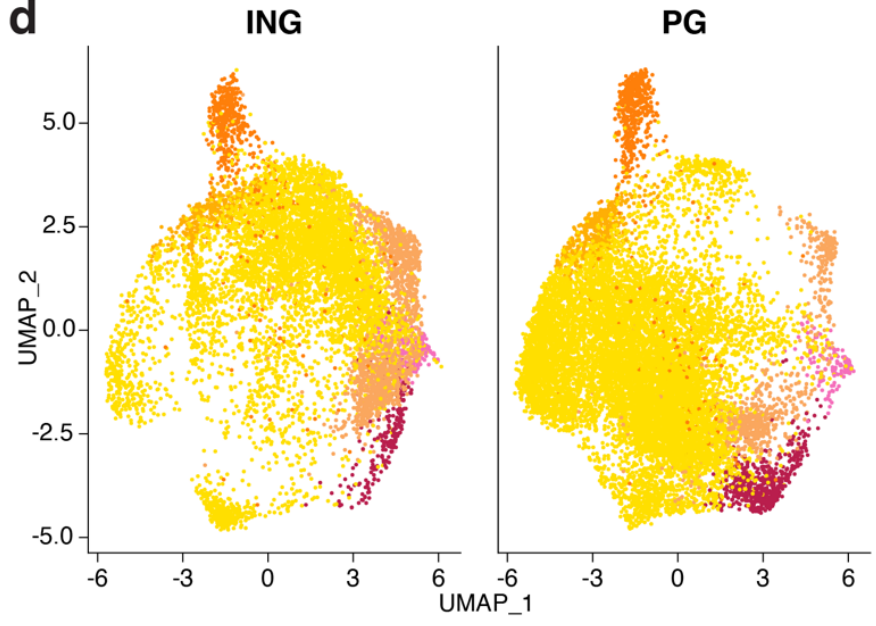

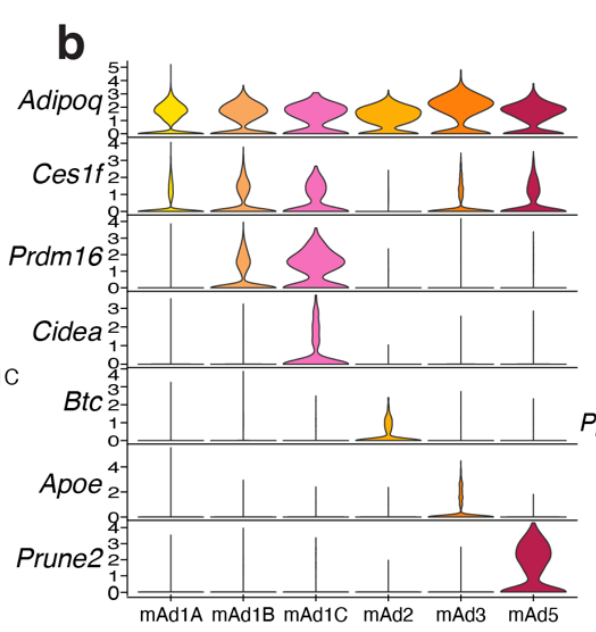

$\mathbf{e}_{1.0}$
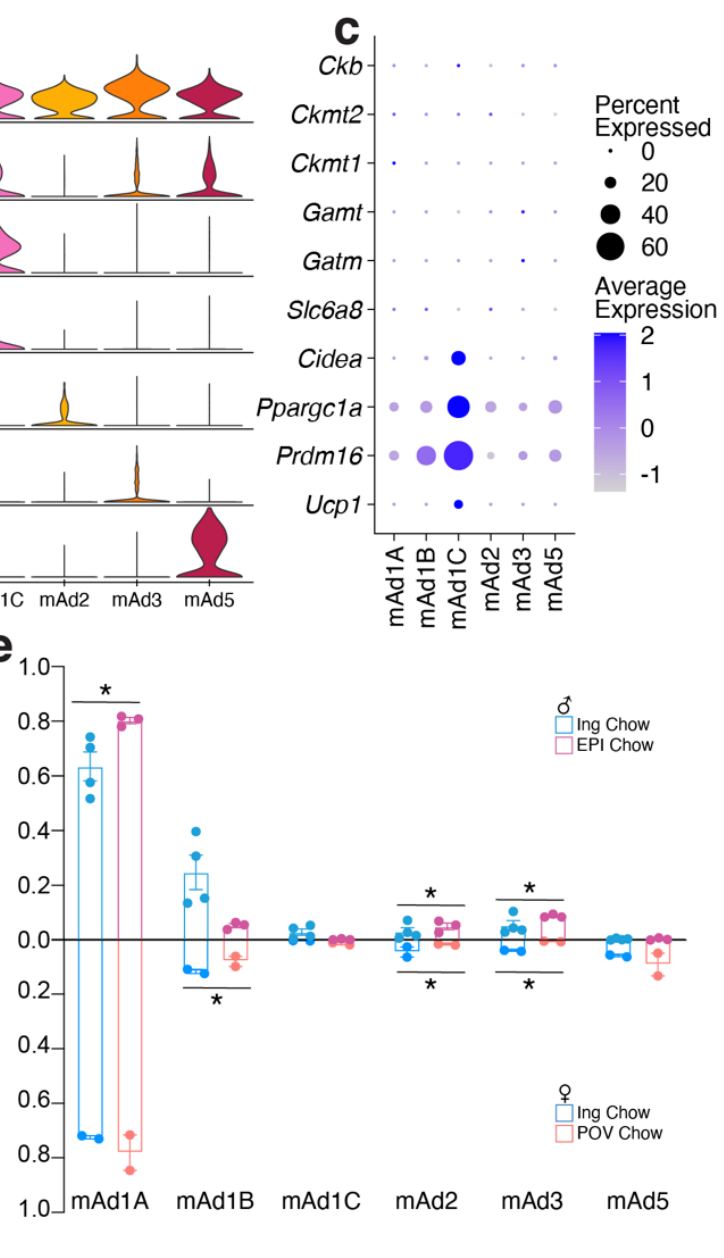

1204 Extended Data Fig. 15. Mouse adipocytes from chow fed animals form a thermogenic

1205 subpopulation. a, UMAP projection of 21,519 adipocytes from chow fed animals. b, Marker

1206 gene expression of adipocytes from chow fed mice. c, Thermogenic gene expression in mouse

1207 chow adipocyte subclusters. d, UMAP projection of adipocytes from chow fed animals split by

1208 depot. e, Proportion of cells in each sample by cluster split by depot and sex. Error bars represent

1209 SEM, * indicates credible depot effect, calculated using mAd5 as reference. 
bioRxiv preprint doi: https://doi.org/10.1101/2021.11.09.466968; this version posted November 11,2021 . The copyright holder for this preprint (which was not certified by peer review) is the author/funder, who has granted bioRxiv a license to display the preprint in perpetuity. It is made available under aCC-BY-NC-ND 4.0 International license.
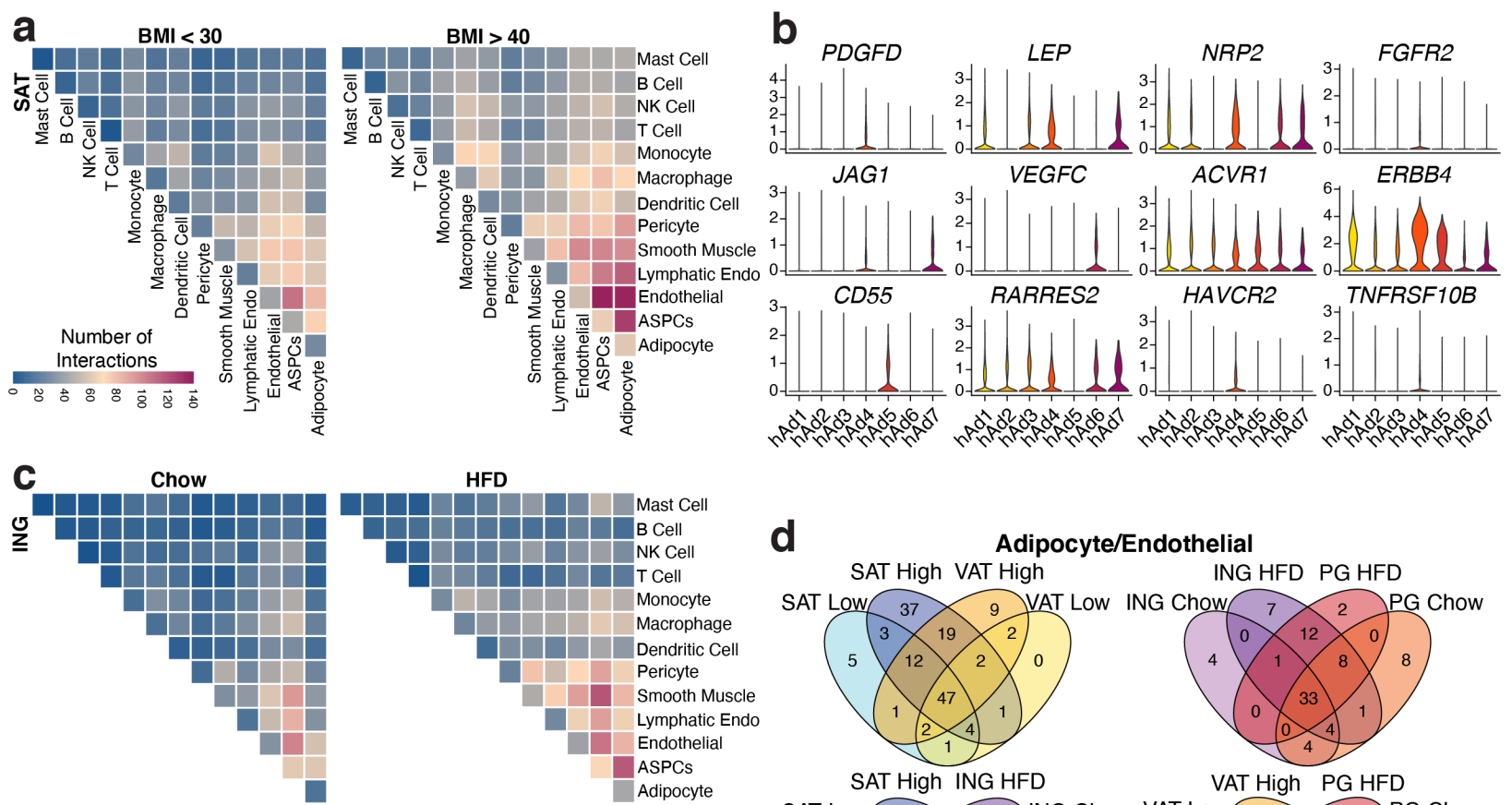

\section{d Adipocyte/Endothelial}

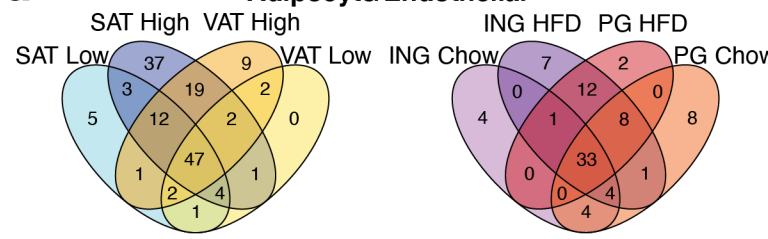

SAT High ING HFD

VAT High PG HFD
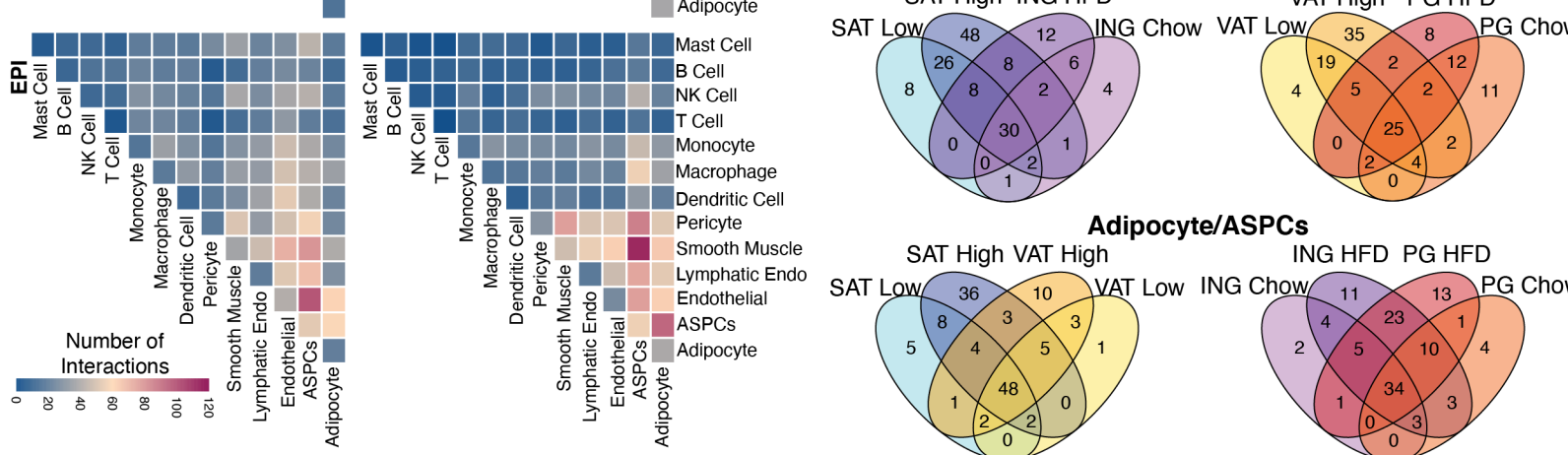

Adipocyte/ASPCs
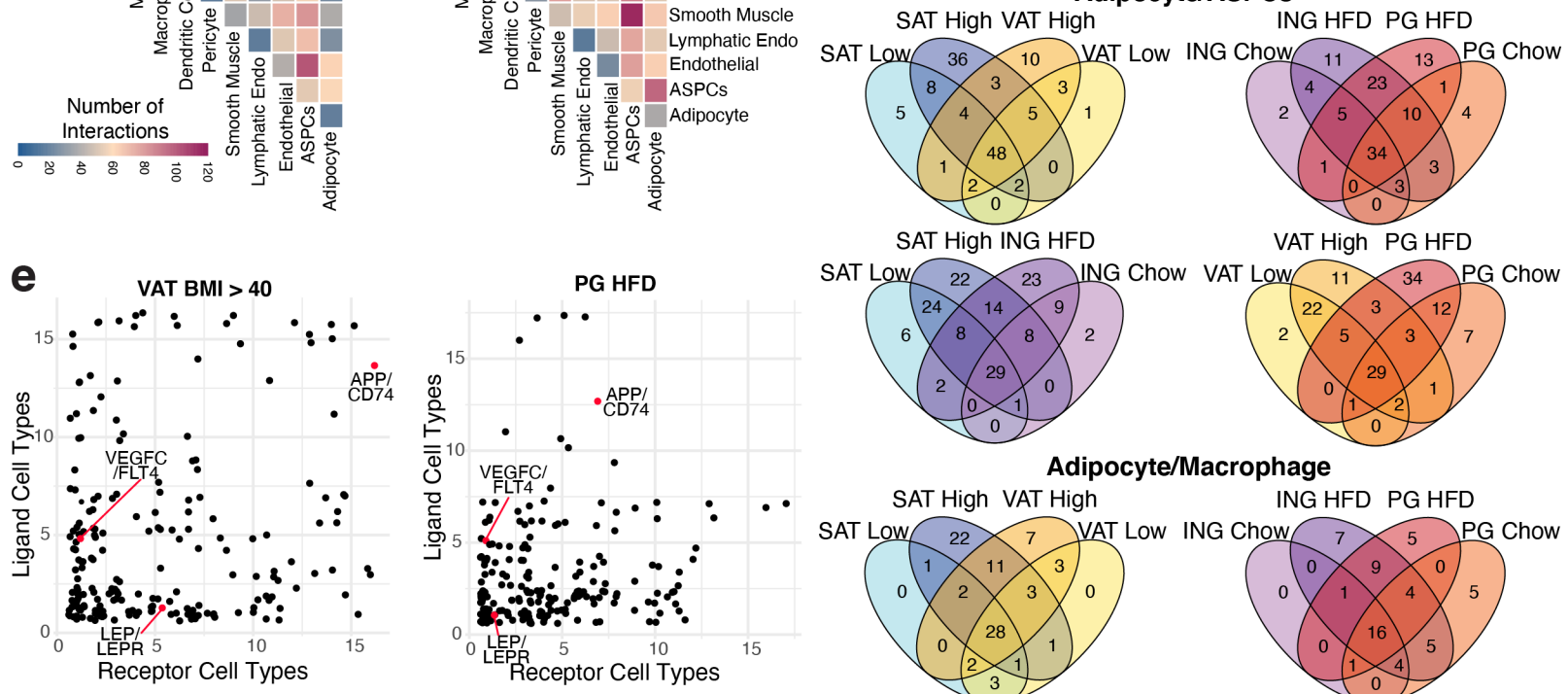

SAT Low 22 ING Chow VAT LOW 23 11 34 PG Chow

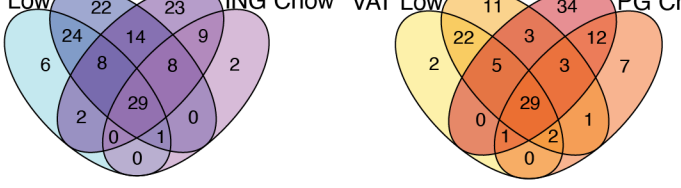

Adipocyte/Macrophage

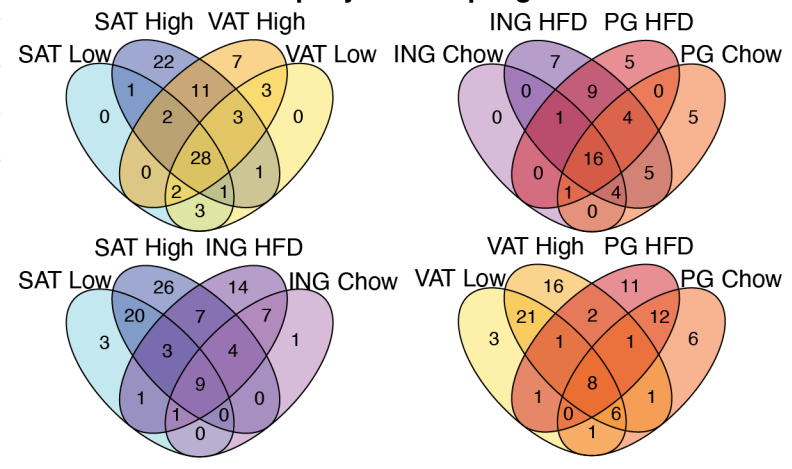

1210 Extended Data Fig. 16. CellphoneDB identifies increasing numbers of cell-cell interactions

1211 within WAT during obesity. a, Heatmap showing number of significant interactions identified 
1212 between cell types in SAT of low $(<30)$ and high $(>40)$ BMI individuals as determined by

1213 CellphoneDB. b, Expression levels of ligand and receptor genes from Figure 4b in human

1214 adipocyte subclusters. c, Heatmaps showing number of significant interactions identified

1215 between cell types in ING and PG WAT of chow and HFD fed mice. d, Venn diagrams showing

1216 the overlap of significant interactions between adipocytes and endothelial cells, ASPCs, and

1217 macrophages between depot, BMI/diet, and species. e, Jitter plots of the relationship between

1218 number of WAT cell types expressing a ligand (y axis) vs. the number of cell types expressing

1219 the receptor (x axis) for all significant interactions in high BMI human VAT (left) and mouse

1220 HFD PG (right). 

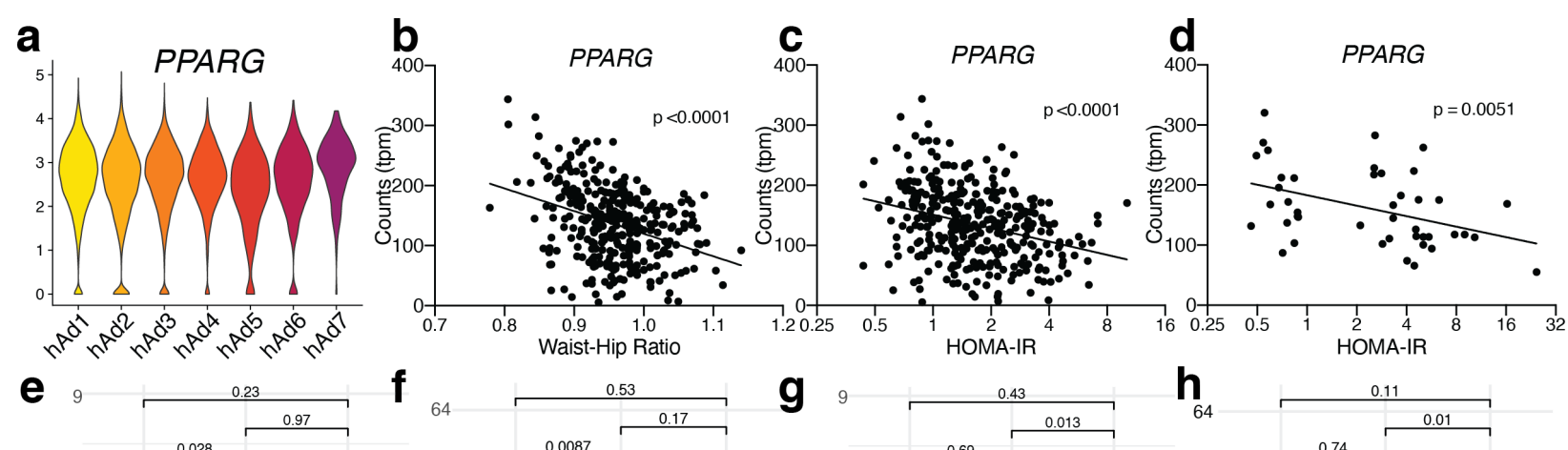

g
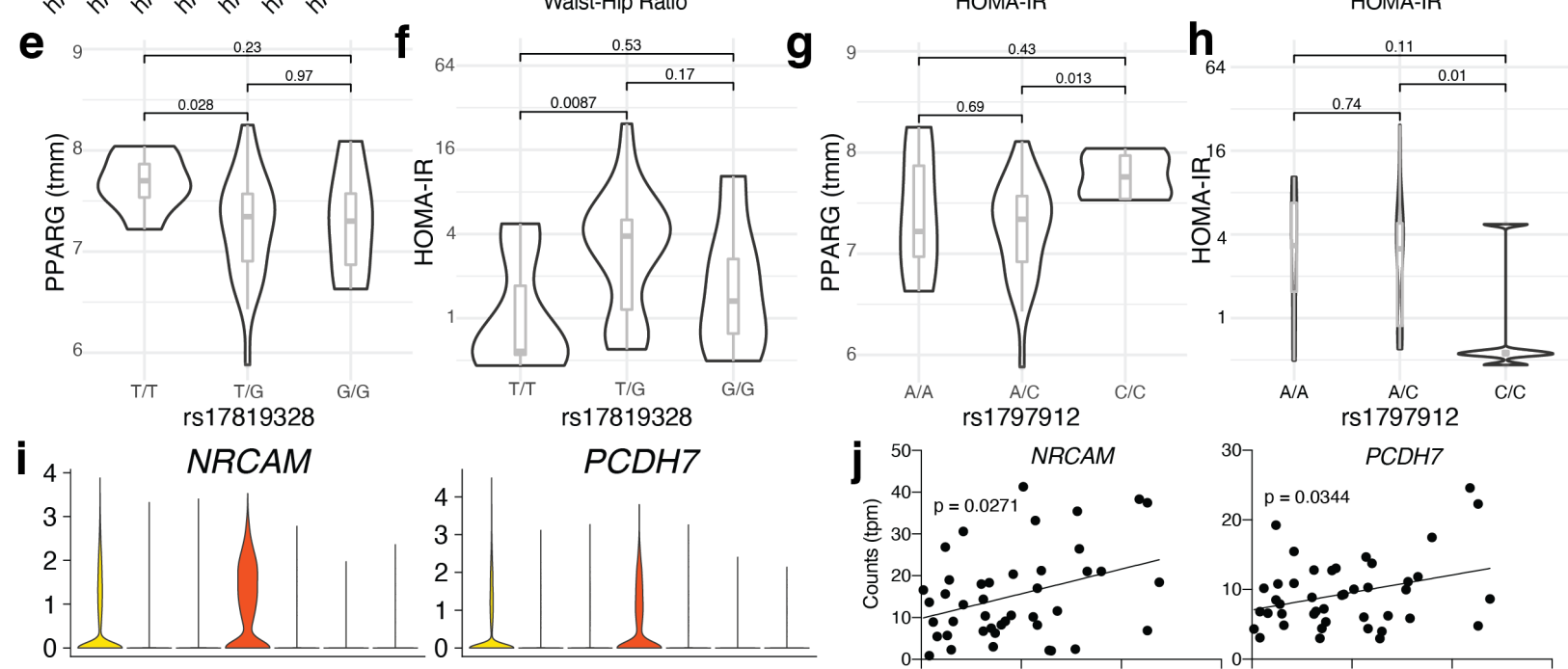

$\mathrm{PCDH7}$
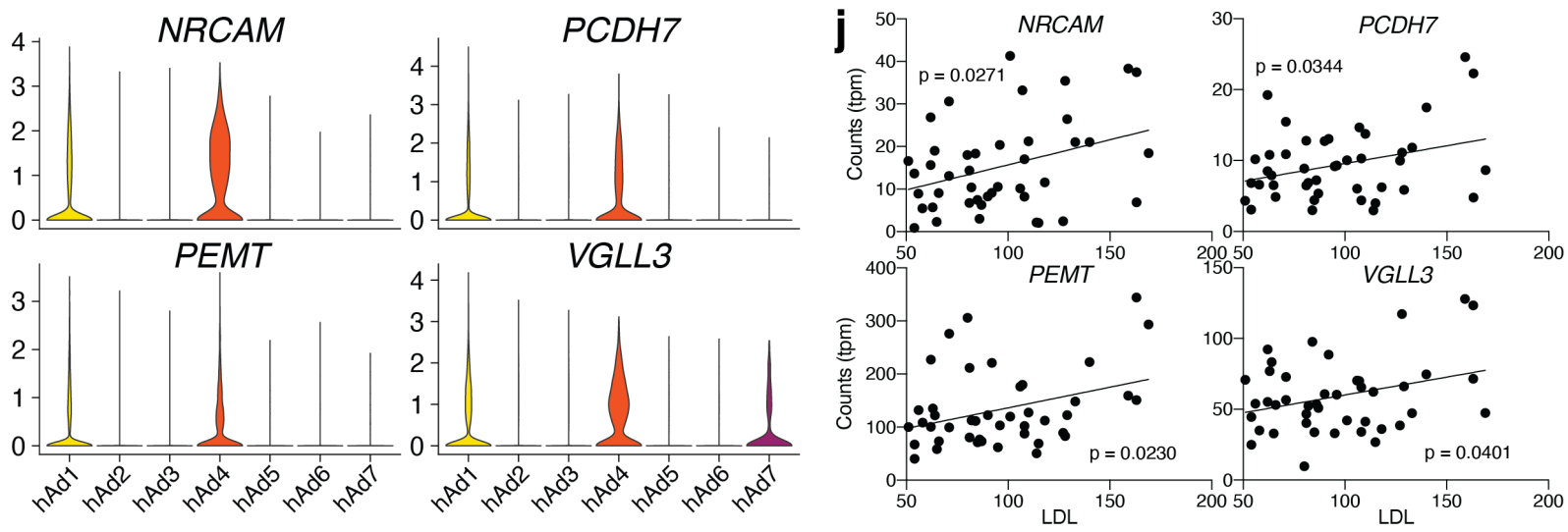

1221 Extended Data Fig. 17. Association with GWAS data provides further insight into the

1225 plotted against HOMA-IR. e-h, SNPs in the PPARG gene identified by DEPICT as associated

1226 with BMI-adjusted WHR plotted against $P P A R G$ gene expression $(\mathbf{e}, \mathbf{g})$ and HOMA-IR $(\mathbf{f}, \mathbf{h})$ in

1227 isolated subcutaneous adipocyte bulk RNA-seq data and cohort. i-j, Expression of genes in

1228 human adipocyte subtypes from sNuc-seq data (i) and from isolated subcutaneous adipocyte bulk 


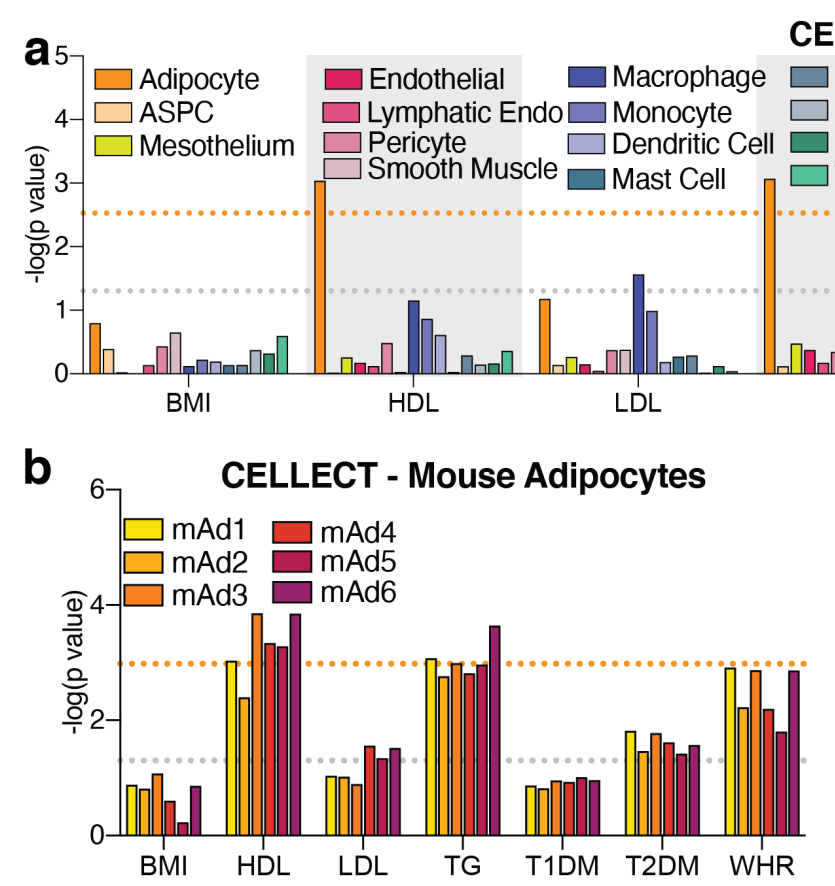

\section{CELLECT}

Neutrophil

B Cell

NK Cell

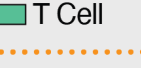

Extended Data Fig. 18. CELLECT identifies mouse cell types associated with human

1231 GWAS studies. a, $p$ values of the association between mouse cell types and GWAS studies. b-c,

$1232 p$ values of the association between mouse adipocyte (b) or ASPC (c) subclusters with GWAS

1233 studies. For all graphs, the grey line represents $p=0.05$ and the orange line represents significant

$1234 p$ value after Bonferroni adjustment ( $p=0.003$ for all cell, $p=0.001$ for subclusters), calculated

1235 based on number of cell types queried. 


\section{Extended Data Table 1. Subject information for Drop-Seq, sNuc-seq, and bulk RNA-seq of}

\section{7 isolated subcutaneous human adipocytes}

Subjects for Drop-Seq

\begin{tabular}{|l|l|l|l|l|l|l|l|}
\hline Subject & BMI & Age & Sex & Race/Ethnicity & SAT & Surgery & Institution \\
\hline Hs235 & 36.04 & 53 & F & Caucasian & Pannus & Panniculectomy & BIDMC \\
\hline Hs236 & 25.74 & 35 & F & Caucasian & Thigh & Thighplasty & BIDMC \\
\hline Hs237 & 22.59 & 53 & F & Caucasian & Pannus & DIEP & BIDMC \\
\hline Hs238 & 19.57 & 49 & F & Caucasian & Pannus & Abdominoplasty & BIDMC \\
\hline Hs239 & 24.8 & 71 & F & Caucasian & Pannus & DIEP & BIDMC \\
\hline Hs240 & 25.82 & 59 & F & Caucasian & Pannus & Panniculectomy & BIDMC \\
\hline Hs242 & 22.88 & 59 & F & Caucasian & Pannus & DIEP & BIDMC \\
\hline Hs248 & 32.28 & 68 & F & Caucasian & Pannus & Panniculectomy & BIDMC \\
\hline Hs249 & 26.46 & 54 & F & Caucasian & Pannus & DIEP & BIDMC \\
\hline
\end{tabular}

DIEP: Deep inferior epigastric perforators

\section{Subjects for sNuc-seq}

\begin{tabular}{|l|l|l|l|l|l|l|l|l|}
\hline Subject & BMI & Age & Sex & $\begin{array}{l}\text { Race/ } \\
\text { Ethnicity }\end{array}$ & SAT & VAT & Surgery & Institution \\
\hline Hs001 & 49.3 & 29 & F & Caucasian & Periumbilical & Omental & VSG & UPitt \\
\hline Hs002 & 33.1 & 57 & F & Caucasian & Periumbilical & NA & Hernia & UPitt \\
\hline Hs004 & 25.4 & 51 & F & Caucasian & Periumbilical & NA & CCY & UPitt \\
\hline Hs009 & 45.7 & 41 & F & Black & Periumbilical & Omental & VSG & UPitt \\
\hline Hs010 & 43.1 & 35 & F & Caucasian & Periumbilical & Omental & RYGB & UPitt \\
\hline Hs011 & 42.8 & 58 & F & Black & Periumbilical & NA & VSG & UPitt \\
\hline Hs012 & 48.7 & 36 & M & Caucasian & Periumbilical & Omental & VSG & UPitt \\
\hline Hs013 & 43.2 & 24 & M & Caucasian & Periumbilical & Omental & VSG & UPitt \\
\hline Hs253 & 30.04 & 53 & F & Caucasian & Periumbilical & Preperitoneal & TAH BSO & BIDMC \\
\hline Hs254 & 23.96 & 41 & F & $\begin{array}{l}\text { Caucasian/ } \\
\text { Hispanic }\end{array}$ & Periumbilical & Preperitoneal & TAH BSO & BIDMC \\
\hline Hs255 & 24.27 & 73 & F & Caucasian & Periumbilical & Preperitoneal & TAH BSO & BIDMC \\
\hline Hs256 & 34.53 & 41 & F & Black & Periumbilical & Omental & CCY & BIDMC \\
\hline Hs266 & 22.15 & 68 & M & Caucasian & Periumbilical & Omental & Colon polyp & BIDMC \\
\hline
\end{tabular}

VSG: Vertical sleeve gastrectomy

CCY: Cholecystectomy

RYGB: Roux en Y gastric bypass

TAH BSO: Total abdominal hysterectomy and bilateral salpingo-oophorectomy

Bulk RNA-seq of floated adipocytes

\begin{tabular}{|l|l|l|l|}
\hline & $\begin{array}{c}\text { Insulin Sensitive } \\
\text { average(min-max) }\end{array}$ & \multicolumn{1}{|c|}{$\begin{array}{c}\text { Insulin Resistant } \\
\text { average(min-max) }\end{array}$} & $\boldsymbol{p}$ Value \\
\hline N & 16 & 27 & \\
\hline AGE & $47.3(36-63)$ & $50.6(33-71)$ & 0.289 \\
\hline BMI & $27.2(21-33)$ & $30.1(21-42)$ & 0.042 \\
\hline HOMA-IR & $0.70(0.46-0.88)$ & $5.8(2.1-24.5)$ & 0.00012 \\
\hline HDL & $70.5(42-154)$ & $54.1(26-100)$ & 0.022 \\
\hline LDL & $93.2(54-133)$ & $97.9(51-169)$ & 0.651 \\
\hline
\end{tabular}




\section{Extended Data Table 2. Numbers of cells in human and mouse single cell experiments}

1239 broken down by cluster, depot, BMI/diet, and technology

Human Cell Numbers

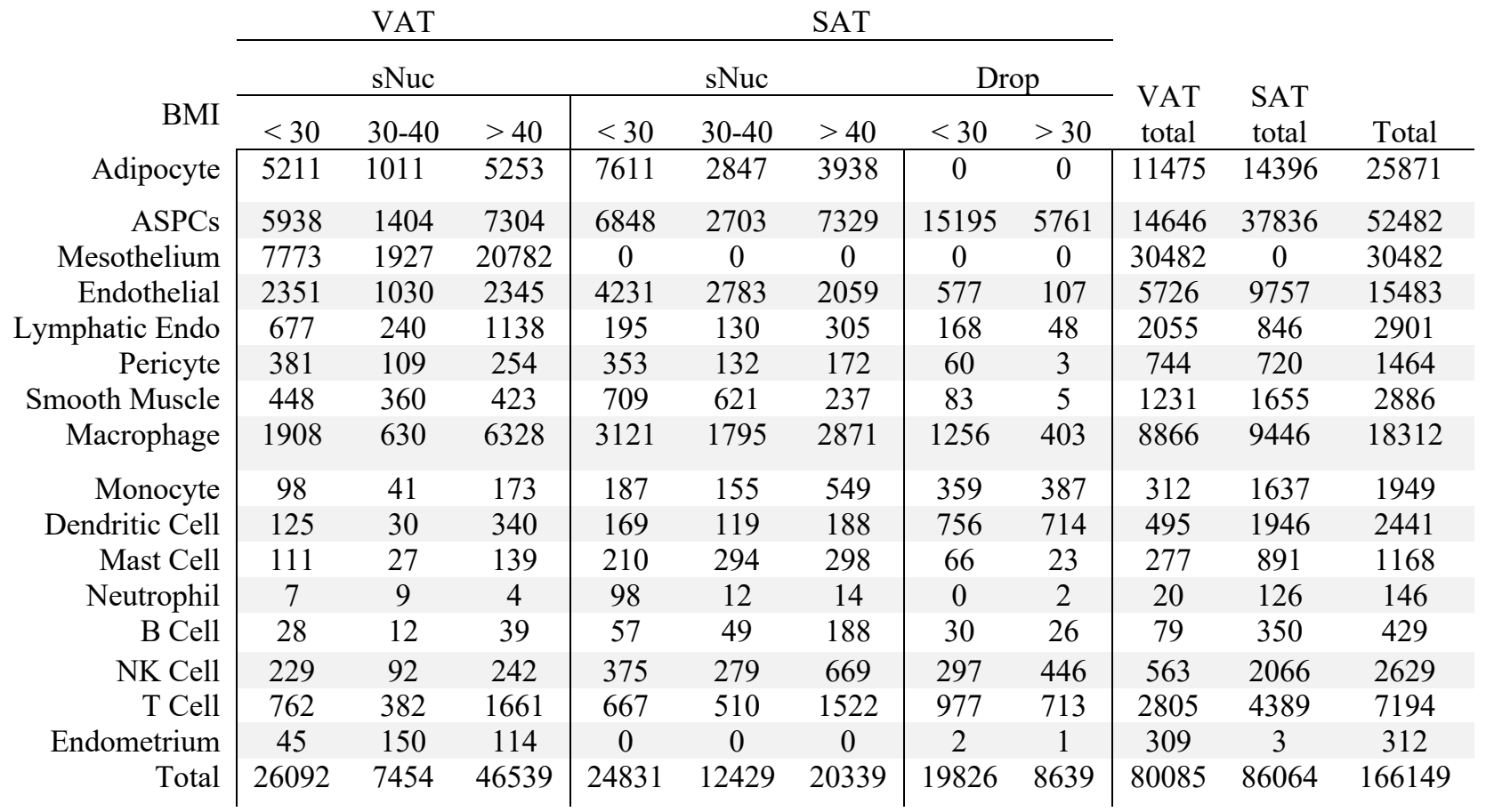

\section{Mouse Cell Numbers}

\begin{tabular}{r|cc|cc|ccc} 
& \multicolumn{2}{c}{ PG } & \multicolumn{2}{c}{ Ing } & & & \\
\cline { 2 - 5 } & Chow & HFD & Chow & HFD & PG Total & Ing Total & Total \\
\cline { 2 - 6 } Adipocyte & 12874 & 5139 & 8645 & 13276 & 18013 & 21921 & 39934 \\
ASPCs & 9928 & 10194 & 16308 & 14797 & 20122 & 31105 & 51227 \\
Mesothelium & 10074 & 4873 & 0 & 0 & 14947 & 0 & 14947 \\
Endothelial & 1521 & 673 & 1141 & 2261 & 2194 & 3402 & 5596 \\
Lymphatic Endo & 678 & 101 & 224 & 173 & 779 & 397 & 1176 \\
Pericyte & 62 & 170 & 56 & 309 & 232 & 365 & 597 \\
Smooth Muscle & 56 & 52 & 30 & 125 & 108 & 155 & 263 \\
Macrophage & 3788 & 35673 & 9370 & 9017 & 39461 & 18387 & 57848 \\
Monocyte & 975 & 2801 & 1286 & 2545 & 3776 & 3831 & 7607 \\
Dendritic Cell & 268 & 688 & 237 & 379 & 956 & 616 & 1572 \\
Mast Cell & 4 & 267 & 13 & 27 & 271 & 40 & 311 \\
Neutrophil & 23 & 9 & 8 & 7 & 32 & 15 & 47 \\
B Cell & 301 & 594 & 28 & 279 & 895 & 307 & 1202 \\
NK Cell & 110 & 215 & 67 & 282 & 325 & 349 & 674 \\
T Cell & 266 & 472 & 69 & 479 & 738 & 548 & 1286 \\
Male Epithelial & 3463 & 36 & 19 & 329 & 3499 & 348 & 3847 \\
Female & 76 & 45 & 6331 & 3135 & 121 & 9466 & 9587 \\
Epithelial & 76 & 4367 & & & & & \\
Total & 44467 & 62002 & 43832 & 47420 & 106469 & 91252 & 197721
\end{tabular}


bioRxiv preprint doi: https://doi.org/10.1101/2021.11.09.466968; this version posted November 11 2021. The copyright holder for this preprint (which was not certified by peer review) is the author/funder, who has granted bioRxiv a license to display the preprint in perpetuity. It is made available under aCC-BY-NC-ND 4.0 International license.

\section{Extended Data Table 3. GWAS studies used for CELLECT analysis}

\begin{tabular}{|ll|}
\hline Trait & $\begin{array}{l}\text { Study/collection } \\
\text { BMI }\end{array}$ \\
$\begin{array}{l}\text { Pulit, S. L. et al. Meta-analysis of genome-wide association studies for body fat } \\
\text { distribution in 694 649 individuals of European ancestry. }\end{array}$ \\
\hline HDL & https://alkesgroup.broadinstitute.org/sumstats formatted/ \\
\hline LDL & $\underline{\text { https://alkesgroup.broadinstitute.org/sumstats formatted// }}$ \\
\hline T1D & https://alkesgroup.broadinstitute.org/sumstats formatted/ \\
\hline $\begin{array}{l}\text { T2D (BMI } \\
\text { adjusted) }\end{array}$ & $\begin{array}{l}\text { Mahajan, A. et al. Fine-mapping type 2 diabetes loci to single-variant resolution using } \\
\text { high-density imputation and islet-specific epigenome maps. }\end{array}$ \\
\hline Triglycerides & https://alkesgroup.broadinstitute.org/sumstats formatted/ \\
\hline $\begin{array}{l}\text { WHR (BMI } \\
\text { adjusted) }\end{array}$ & $\begin{array}{l}\text { Loh, P.-R., Kichaev, G., Gazal, S., Schoech, A. P. \& Price, A. L. Mixed-model } \\
\text { association for biobank-scale datasets }\end{array}$ \\
\hline
\end{tabular}

